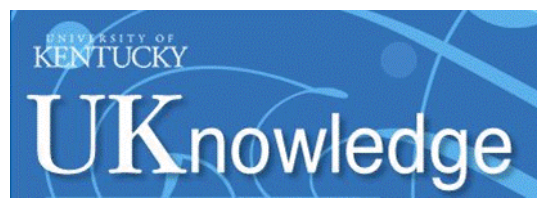

University of Kentucky

UKnowledge

Theses and Dissertations--Electrical and Computer Engineering

2015

\title{
THE CONSTRAINED LOCALLY CORRECTED NYSTRÖM METHOD
}

Nastaran Hendijani

University of Kentucky, nastaran.hendijani@gmail.com

Right click to open a feedback form in a new tab to let us know how this document benefits you.

\section{Recommended Citation}

Hendijani, Nastaran, "THE CONSTRAINED LOCALLY CORRECTED NYSTRÖM METHOD" (2015). Theses and Dissertations--Electrical and Computer Engineering. 68.

https://uknowledge.uky.edu/ece_etds/68

This Doctoral Dissertation is brought to you for free and open access by the Electrical and Computer Engineering at UKnowledge. It has been accepted for inclusion in Theses and Dissertations--Electrical and Computer Engineering by an authorized administrator of UKnowledge. For more information, please contact UKnowledge@lsv.uky.edu. 


\section{STUDENT AGREEMENT:}

I represent that my thesis or dissertation and abstract are my original work. Proper attribution has been given to all outside sources. I understand that I am solely responsible for obtaining any needed copyright permissions. I have obtained needed written permission statement(s) from the owner(s) of each third-party copyrighted matter to be included in my work, allowing electronic distribution (if such use is not permitted by the fair use doctrine) which will be submitted to UKnowledge as Additional File.

I hereby grant to The University of Kentucky and its agents the irrevocable, non-exclusive, and royalty-free license to archive and make accessible my work in whole or in part in all forms of media, now or hereafter known. I agree that the document mentioned above may be made available immediately for worldwide access unless an embargo applies.

I retain all other ownership rights to the copyright of my work. I also retain the right to use in future works (such as articles or books) all or part of my work. I understand that I am free to register the copyright to my work.

\section{REVIEW, APPROVAL AND ACCEPTANCE}

The document mentioned above has been reviewed and accepted by the student's advisor, on behalf of the advisory committee, and by the Director of Graduate Studies (DGS), on behalf of the program; we verify that this is the final, approved version of the student's thesis including all changes required by the advisory committee. The undersigned agree to abide by the statements above.

Nastaran Hendijani, Student

Dr. Robert J. Adams, Major Professor

Dr. Cai-Cheng Lu, Director of Graduate Studies 
THE CONSTRAINED LOCALLY CORRECTED NYSTRÖM METHOD

\title{
DISSERTATION
}

A dissertation submitted in partial fulfillment of the requirements for the degree of Doctor of Philosophy in the College of Engineering at the University of Kentucky

\author{
By \\ Nastaran Hendijani \\ Lexington, Kentucky
}

Director: Dr. Robert J. Adams, Associate Professor of

Electrical and Computer Engineering

Lexington, Kentucky

2015

Copyright (C) Nastaran Hendijani 2015 


\section{ABSTRACT OF DISSERTATION}

\section{THE CONSTRAINED LOCALLY CORRECTED NYSTRÖM METHOD}

In this dissertation a generalization of the locally corrected Nyström (LCN) discretization method is outlined wherein sparse transformations of the LCN system matrix are obtained via singular value decompositions of local constraint matrices. The local constraint matrices are used to impose normal continuity of the currents across boundaries shared by mesh elements. For this reason, the method is called constrained LCN (CLCN).

Due to the CLCN's simplicity and flexibility, it is straightforward to develop high order CLCN systems for different formulations and mesh element types. As compared to the LCN, the CLCN method offers memory savings and improved accuracy when applied to geometries with sharp edges. Furthermore, the CLCN method maintains the high-order convergence of the LCN method, and it eliminates the need to include line charges in Nyström-based discretizations of formulations that involve the continuity equation.

In addition to developing the CLCN method, we will investigate a high-order Nyström implementation of an augmented VIE called AVIE as an alternative to the VIE formulation. The limitations of VIE include poor matrix condition numbers for problems with high contrast materials, and deteriorating performance for problems with complex, multi-scale meshes.

The AVIE formulation incorporates surface and volume charges as additional unknowns and includes both current continuity and charge neutrality constraints. It is found that a straightforward LCN implementation of the AVIE formulation is still poorly conditioned in some cases. However, it will be shown that the AVIE formulation provides sufficient flexibility to enable the resulting linear system to be appropriately scaled in order to significantly improve the matrix condition numbers. 
KEYWORDS: locally corrected Nyström method, method of moments, normal continuity, transformation

Nastaran Hendijani

Student's Signature

April 23, 2014

Date 


\title{
CONSTRAINED LOCALLY CORRECTED NYSTRÖM METHOD
}

\author{
By
}

Nastaran Hendijani

Robert J. Adams

Director of Dissertation

Cai-Cheng Lu

Director of Graduate Studies

April 23, 2014 
To my parents who have always encouraged me to reach the highest values 


\section{ACKNOWLEDGMENTS}

I acknowledge with massive gratitude the support and assistance of my advisor Dr. Robert J. Adams during my PhD studies in the University of Kentucky. With his brilliant ideas and thoughtful guidance he always helped me to overcome the obstacles I encountered in doing research. He taught me how to think critically and boost selfconfidence to develop novel ideas.

I would like to thank Dr. John C. Young for his patience in answering my questions and providing his meticulous insights and precious suggestions. Moreover, I had the opportunity to learn from his prominent skills and experience in computer programming.

I am very thankful to Prof. Stephen D. Gedney for his valuable observations and suggestions and providing the original HO LCN code. In addition, taking his computational electromagnetic courses was a major step in developing my understanding of this area.

I would like to thank Prof. Gary S. Brown at Virginia Tech who provided me with an inspiring and distinctive view of the electromagnetics and motivated me with his encouraging remarks during my $\mathrm{PhD}$ studies.

I would like to express my thanks to Prof. Cai-Cheng Lu for his time to evaluate my research as a committee member and also his assistance and cooperation in solving the issues I had related to transferring from Virginia Tech. Moreover, I have to thank Prof. Qiang Ye for his time to serve as my committee member.

I am very grateful to Dr. Jin Cheng for her patience in helping me to develop the necessary skills to initiate my research in the University of Kentucky. I benefitted greatly from her knowledge and experience. Furthermore, as a friend she was very sympathetic and cooperative when I faced difficulties.

I wish to thank Prof. Homayoon Oraizi for his guidance, support, and encouragement. I have to thank my friends Ms. Farzane Noormohammadi, Dr. Vahid Khalilzad-Sharghi, Mr. Hossein Hadidian-Moghadam, Mr. Hossein Tamjid, Dr. Jun-shik Choi, and Dr. Taeyoung Yang for helping me and inspiring me to pursue my $\mathrm{PhD}$ studies. 
Finally, I would like to thank my parents, sisters, and husband for their patience, support, and endless love. 


\section{Table of Contents}

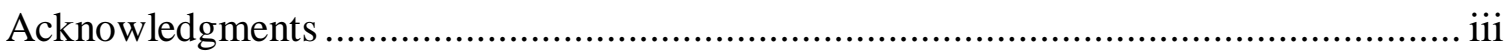

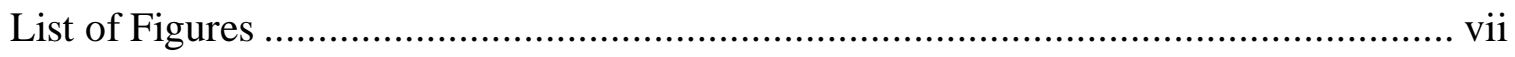

Chapter 1. Introduction ..................................................................................

1.1. Overview and Motivation..................................................................

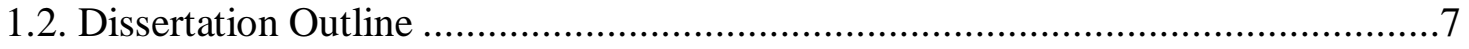

Chapter 2. Integral Equation Methods ...............................................................

2.1. Maxwell's Equations and Equivalence Principles..........................................10

2.2. Formulating the Scattering Problem .........................................................14

2.3. L and K Operators.............................................................................20

2.4. The Frequency Instability of the EFIE...................................................21

Chapter 3. The Locally Corrected Nyström Method.................................................24

3.1. The Original Nyström Method ..........................................................24

3.2. Local Corrections for Singular Kernels .................................................26

3.3. LCN Applied to Electromagnetic Integral Equations ......................................28

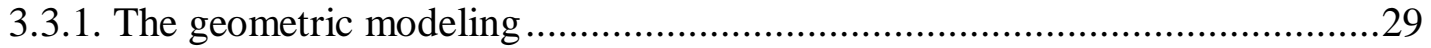

3.3.2. Discretization of the integral operator.....................................................32

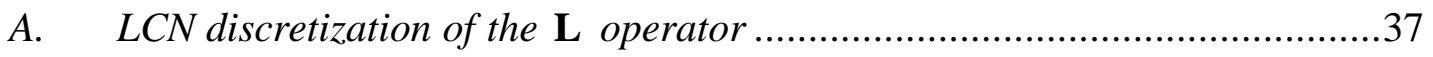

B. LCN discretization of the $\mathbf{K}$ operator.................................................42

3.4. The Basis Functions for Local Corrections ...................................................44

3.5. LCN Test and Source Scheme..............................................................47

3.6. Selecting LCN Test Vectors.................................................................49

Chapter 4. The Augmented VIE Formulation...........................................................50

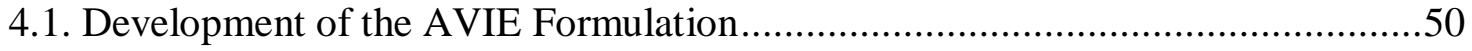

4.2. The Iterative Diagonal Scaling Algorithm ..............................................54 


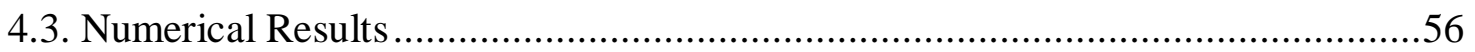

Chapter 5. The Constrained LCN Discretization Method ................................................69

5.1. Connection of LCN to the Method of Moment .................................................

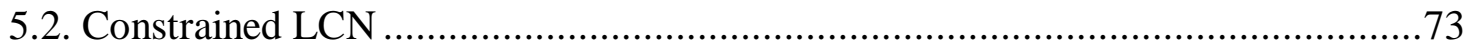

5.2.1. Transformation vectors on individual cells ...................................................74

5.2.2. Transformation vectors associated with edges ..............................................77

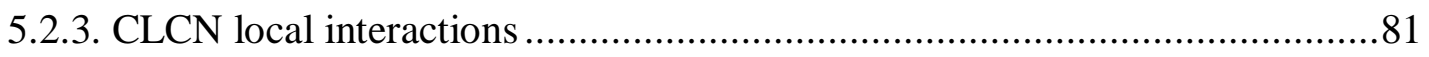

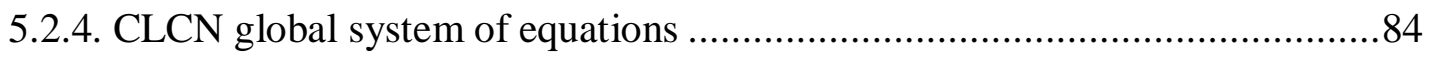

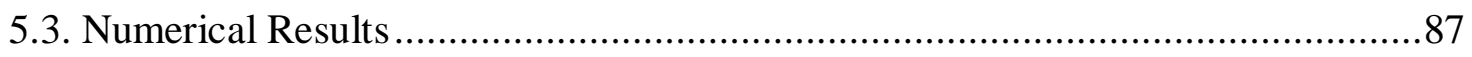

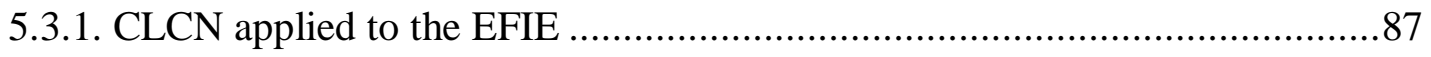

5.3.2. CLCN applied to the augmented EFIE............................................................89

5.3.3. CLCN applied to the MFIE ……………….................................................94

Chapter 6. Conclusions ...............................................................................................107

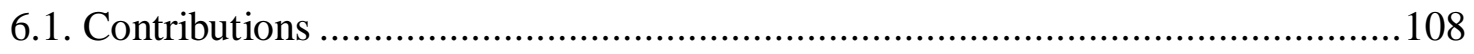

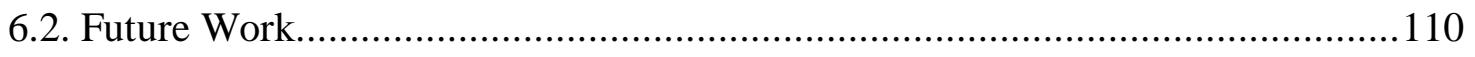

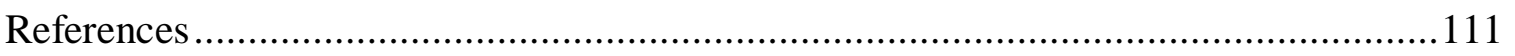

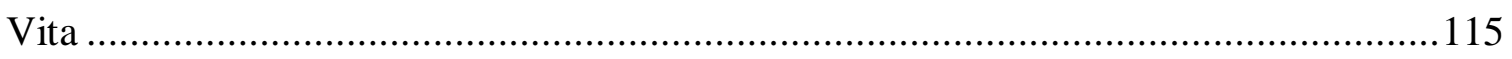




\section{List of Figures}

Figure 1. Surface equivalence principle (a) original problem (b) exterior equivalent problem (c) interior equivalent problem

Figure 2: The condition numbers of the LCN discretization of the AVIE and VIE formulations with iterative scaling applied to $1 \mathrm{~m}$ radius dielectric sphere with different mesh sizes versus the relative permittivity at $1 \mathrm{~Hz}$

Figure 3: The convergence of the right errors $\operatorname{err}_{\mathbf{R}}$ defined in the iterative scaling algorithm applied to the AVIE formulation for scattering from the one meter radius

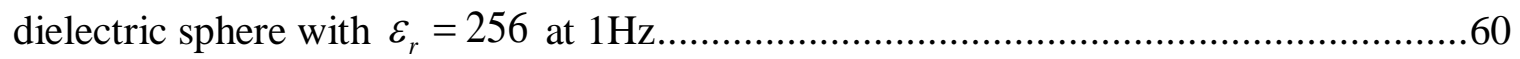
Figure 4: The convergence of the left errors $\operatorname{err}_{\mathrm{L}}$ defined in the iterative scaling algorithm applied to the AVIE formulation for scattering from the one meter radius dielectric sphere with $\varepsilon_{r}=256$ at $1 \mathrm{~Hz}$.

Figure 5: The convergence of the right errors $\operatorname{err}_{\mathbf{R}}$ defined in the iterative scaling algorithm applied to the AVIE formulation for scattering from the one meter radius dielectric sphere with $\varepsilon_{r}=1000$ at $1 \mathrm{~Hz}$

Figure 6: The convergence of the left errors $\operatorname{err}_{\mathrm{L}}$ defined in the iterative scaling algorithm applied to the AVIE formulation for scattering from the one meter radius dielectric sphere with $\varepsilon_{r}=1000$ at $1 \mathrm{~Hz}$

Figure 7: The high order (HO) convergence of the error in near electric and magnetic fields at $1 \mathrm{~Hz}$ computed by the AVIE formulation applied to the $1 \mathrm{~m}$ radius dielectric sphere for different values of $\varepsilon_{r}$

Figure 8: The high order (HO) convergence of the error in radar cross section (RCS) computed by the AVIE for the $1 \mathrm{~m}$ radius dielectric sphere with $\varepsilon_{r}=16$ at $50 \mathrm{MHz} . \ldots \ldots . . .65$

Figure 9: the error of near electric fields at $1 \mathrm{~Hz}$ versus relative permittivity for a $1 \mathrm{~m}$ radius dielectric sphere computed by AVIE and VIE formulations with LOGOS. 
Figure 10: The eigenvalues of the preconditioned AVIE system matrix using simultaneous left and right diagonal scaling algorithm for scattering from the dielectric sphere with $\varepsilon_{r}=400$ at $1 \mathrm{~Hz}$

Figure 11: The eigenvalues of the preconditioned VIE system matrix using simultaneous left and right diagonal scaling algorithm for scattering from the dielectric sphere with $\varepsilon_{r}=400$ at $1 \mathrm{~Hz}$

Figure 12: RMS error of the bi-static RCS for the 1-meter radius PEC sphere at $50 \mathrm{MHz}$ and $300 \mathrm{MHz}$ versus the basis order. .96

Figure 13: Condition numbers for the 1-meter radius PEC sphere at $50 \mathrm{MHz}$ and $300 \mathrm{MHz}$ versus the basis order. .97

Figure 14: Radar cross-sections for scattering from a PEC cube with a 1-meter edge length at $50 \mathrm{MHz}$ in the $\phi=0$ plane. .98

Figure 15: Radar cross-sections for scattering from a PEC corner reflector at $50 \mathrm{MHz}$ in the $\phi=0^{\circ}$ plane.

Figure 16: RMS error of the bi-static RCS for the 1-meter radius PEC sphere at $300 \mathrm{MHz}$ versus the basis order. In the legend, NQL indicates no line charge. .100 Figure 17: RMS error of the near electric field for the 1-meter radius PEC sphere at $1 \mathrm{~Hz}$ versus basis order $p$. The near field is sampled $0.5 \mathrm{~m}$ above the PEC surface. In the legend, NQL indicates no line charge. 101 Figure 18: Condition numbers of the CLCN and LCN system matrices obtained for the AEFIE applied to a 1-meter radius PEC sphere at $300 \mathrm{MHz}$ and $1 \mathrm{~Hz}$ versus the basis order. .102

Figure 19: Radar cross-sections for scattering from a PEC corner reflector at $50 \mathrm{MHz}$ in the $\phi=0$ plane .103

Figure 20: Near electric field for the corner reflector at $1 \mathrm{~Hz}$ sampled on a two meter radius semi-circle in the $\phi=0$ plane .104

Figure 21: RMS error of the bi-static RCS for the 1-meter radius PEC sphere at $200 \mathrm{MHz}$ versus the basis order. .105

Figure 22: Condition numbers of the CLCN and LCN system matrices obtained for the MFIE applied to a 1-meter radius PEC sphere at 200MHz versus the basis order. .106 


\section{Chapter 1. INTRODUCTION}

\subsection{Overview and Motivation}

The electromagnetic theory involves the solution of the Maxwell's equations to find the electromagnetic fields within an object or scattered by the object in a background material. As a consequence, solution techniques that are accurate and stable by varying material properties and frequency are crucial in this area. For a time-harmonic electromagnetic incident wave, one can obtain vector Helmholtz partial differential equations (PDE) by combining Maxwell's equations. As an alternative, the application of the equivalence theorem gives rise to the integral equation (IE) formulations [1]. This is accomplished via replacing the object by equivalent induced currents and/or charges. There are two types of equivalence principle, i.e., volumetric equivalence principle and surface equivalence principle which result in the respective volume and surface integral equations. Surface integral equations (SIE) provide a particularly convenient approach for time harmonic modeling of linear homogeneous materials since representations of the geometry and the unknowns are required only on the surface of a given object. However, for nonlinear, high contrast and/or inhomogeneous materials, volume integral equations (VIEs) often provide a more useful alternative.

In general, the resulting time-harmonic electromagnetic equations, either differential or integral equation, are not analytically solvable. As a consequence, discretization techniques are applied to cast the continuous linear operators to a discrete form, so that the analytical formulations are represented by matrix equations. Discretization methods either have an element-based or a point-based scheme. The element-based methods 
approximate the unknown quantity as sum of a number of basis functions weighted by unknown coefficients [1]. Finite element method (FEM) [2, 3] and method of moments (MOM) [4] are two element-based methods applied to discretize differential equations and integral equations, respectively. Compared to partial differential equation (PDE) methods, integral equation approaches involve smaller number of degrees of freedom (DOFs). Moreover, because of the bounded property of integral equation operator, integral equation methods provide better conditioned system matrices. However, nonlocality of integral equation operators results in dense system matrices; consequently, applications involving a large number of DOFs typically incorporate methods to obtain a sparse representation of the system matrix to improve their overall computational efficiency and memory usage [5-10].

Integral equation methods have been widely applied to solve electromagnetic radiation and scattering problems [1, 11]. Consider the electromagnetic integral equation:

$$
\mathcal{L} f=g,
$$

where, $\mathcal{L}$ is the linear operator. $f$ is the unknown vector quantity, and $g$ is the excitation. Utilizing the MOM, $f$ is expanded in terms of a set of $N$ appropriate basis functions $f_{n}:$

$$
f=\sum_{n=1}^{N} c_{n} f_{n},
$$

where, $c_{n}$ are the unknown basis function coefficients. Substituting (1.2) in (1.1) gives:

$$
\sum_{n=1}^{N} c_{n} \mathcal{L} f_{n}=g,
$$


Applying a set of test functions $t_{m}, m=1, \ldots, N$, in the range of $\mathcal{L}$ and computing their inner product with right and left sides of the above equation, we have:

$$
\sum_{n=1}^{N} c_{n}\left\langle t_{m}, \mathcal{L} f_{n}\right\rangle=\left\langle t_{m}, g\right\rangle
$$

Using the $N$ test functions, the collection of equations in (1.4) can be expressed as a matrix equation:

$$
[\mathbf{Z}] \cdot \overline{\mathbf{c}}=\overline{\mathbf{g}}
$$

where, $[\mathbf{Z}]$ is the $N \times N$ dense MOM impedance matrix with $(m, n)$ th entry $[\mathbf{Z}]_{m, n}=\left\langle t_{m}, \mathcal{L} f_{n}\right\rangle \cdot \overline{\mathbf{c}}$ is the $N \times 1$ vector including the unknown coefficients $c_{n} \cdot \overline{\mathbf{g}}$ is an $N \times 1$ vector with $m$ th entry $(\overline{\mathbf{g}})_{m}=\left\langle t_{m}, g\right\rangle$. Direct or iterative solution methods can be applied to solve (1.5). Direct solution methods compute the inverse of [Z]commonly by the application of factorization algorithms such as LU decomposition or the Cholesky factorization [12, 13]. Iterative methods calculate a set of approximate solutions that converge to the exact solution through the solver iterations. Some instances of iterative solution methods include the conjugate gradient (CG), the bi-conjugate gradient stabilized (Bi-CGSTAB) [14], and the generalized minimal residual (GMRES) method [15]. Direct solution methods increase the memory usage compared to iterative solvers, since they require the factorization of the system matrix. However, the iterative methods are more sensitive to the eigenvalue pattern and the condition number of the system matrix. The number of iterations and consequently the solution time is increased for problems with ill-conditioned system matrices. For $N$ unknowns, the solution time of the MOM is proportional to $N^{3}$ when a dense direct solver is used. This is a result of the 
$O\left(N^{2}\right)$ computational costs of the double integrations appearing in the system matrix fill. Applying iterative solvers the time would be proportional to $\alpha N^{2}$ where $\alpha$ is the number of iterations. By utilizing sparse fast solvers the cost of operations can be reduced to $O(N \log N)[16,17]$.

To increase the computational efficiency, high-order (HO) discretization methods have been developed which provide exponential convergence while reducing the number of DOFs. For the MOM this is achieved through defining HO basis functions [16, 18-21]. However, the implementation of the $\mathrm{HO}$ discretization methods requires $\mathrm{HO}$ curvilinear meshing of the underlying geometry and also integration rules with controllable accuracy.

The high-order (HO) locally corrected Nyström (LCN) method [22-25] has been introduced as an alternative to the more traditional method of moments (MOM) [1, 26, 27] for discretizing integral equations. The point-based LCN discretization offers a number of advantages over methods that rely on HO MOM discretizations. The HO LCN method can considerably reduce the computational costs incurred by high-order MOM methods by avoiding double integrations [23]. Moreover, for large applications that call for the use of sparse solution methods, the point-based structure of the LCN discretization can provide more flexibility in constructing the required multi-level geometric partition [28].

A potential disadvantage of the LCN discretization is that it does not explicitly impose normal continuity on currents that flow across interfaces shared by cells of the mesh. In contrast, the divergence conforming basis functions commonly used in the MOM are constructed to directly impose normal continuity on the source and field quantities across shared cell interfaces $[18,19,26]$. As a result, a low-order (LO) MOM often provides more accurate solutions than a comparable LO LCN method even though 
fewer DOFs are used [29, 30]. The relative inaccuracy of the LO LCN solutions is most evident for objects that possess geometric singularities. In addition, basis discontinuities in the LCN method require the incorporation of line integrals and/or line charges in the discretization of electric field integral equation (EFIE) related formulations for perfect electrical conductors (PECs). For instance, line integrals are required in LCN discretizations of the EFIE operator [23], and line charges are included as additional unknowns in developing augmented EFIE formulations [31].

It has recently been shown that it is possible to map the first-order LCN method for the electric field integral equation into the standard Rao-Wilton-Glisson (RWG) discretization through an appropriate linear transformation [30]. This is accomplished using a detailed analysis of the LCN bases in order to identify a transformation matrix that constructs the RWG basis functions from the first-order polynomial complete LCN basis functions. This approach both reduces the size of the resulting system matrix relative to the initial LCN matrix and improves the accuracy of the resulting LCN solutions. Extensions of the method to high order discretizations and/or different mesh elements appears to be nontrivial, requiring specific treatments for each new situation.

In the following, we outline a method that has somewhat similar goals, but which uses a more general approach. The new discretization is referred to as the constrained LCN (CLCN) method and is based on the imposition of auxiliary continuity constraints on the underlying LCN basis space. The auxiliary constraints are first imposed locally on individual cells of the mesh, and then on pairs of cells that share an interface. In both cases, simple algebraic manipulations of the continuity constraints lead to a set of 
transformations that provide normal continuity of vector quantities between neighboring cells of the mesh for a given HO LCN discretization.

Whereas the approach of [30] generates an equivalence between a MOM method and an LCN-based discretization, the proposed CLCN method discussed here does not attempt to mimic a particular MOM discretization. Instead, a set of continuity constraints are imposed on the initial LCN basis, and linear algebraic operations are used to construct appropriate basis subspaces. The functional forms of the underlying CLCN basis functions are not explicitly specified. This avoids some of the complications encountered in building HO MOM basis functions [18, 19].

From a global point of view, the CLCN method introduces two sparse transformation matrices, which multiply the global LCN impedance matrix from the left and the right to produce the CLCN system matrix. It is shown that the CLCN matrix is smaller than the LCN matrix, with the reduction factor depending on the order of the underlying LCN discretization. In the case of a zeroth-order LCN method for a closed surface using mixed-order current basis functions on quadrilaterals, the CLCN matrix is four times smaller than the original LCN matrix.

The CLCN method maintains the high-order convergence of the LCN method for smooth geometries and provides more accurate solutions than the LCN method for problems involving singular geometries. Moreover, the method eliminates the need to include line charge DOFs in the formulation. This is validated by applying the CLCN method to the augmented EFIE (AEFIE) formulation [31].

In addition to introducing the CLCN method, the augmented VIE (AVIE) formulation is developed. As mentioned before, the VIE approach is an appropriate 
formulation for problems involving nonlinear or inhomogeneous materials. Moreover, by applying the LCN discretization to the VIE formulation, the material properties can be confined to the diagonal of the system matrix which significantly reduces the computational costs in cases for which a given geometry with different material properties is of interest. However, the dependence of the diagonal of the LCN discretized VIE to the material specifications results in high condition numbers of the system matrix for high contrast materials at low frequencies [32, 33].

By introducing surface and volume charges as additional unknowns and incorporating current continuity and charge neutrality constraints, the AVIE formulation offers system condition numbers independent of the material properties at low frequencies. Moreover, in order to further improve the condition numbers of the AVIE formulation an iterative scaling method is utilized [34].

\subsection{Dissertation Outline}

Chapter 2 represents the fundamental electromagnetic integral equations. The surface integral equations (SIEs) for perfect electric conductors (PECs) and volume integral equations (VIEs) for penetrable materials are derived by the application of surface and volumetric equivalence theorems. The advantages and disadvantages of different methods will be discussed.

Chapter3 describes the LCN method to solve integral equations. Starting from the conventional Nyström method for the regular integrands, we introduce the local corrections that are the key to the application of the Nyström method for integral equations with singular kernels. Then, LCN discretizations of the surface and volume 
integral equations are demonstrated. The practical issues regarding the choice of the basis functions and test vectors are also discussed.

Chapter 4 exhibits the implementation of the AVIE formulation which is based on the VIE formulation and incorporates surface and volume charge continuity constrains. An iterative diagonal scaling preconditioner is described to improve the conditioning of the AVIE formulation. It is shown the preconditioned AVIE system matrix provides stable condition numbers by increasing the material contrast at low frequencies.

Finally, chapter 5 introduces the constrained LCN (CLCN) method which applies local constraints to impose the normal continuity of source and test vector quantities to the LCN discretization. Development of the CLCN transformation vectors based on singular value decomposition (SVD) of the local constraint matrices are given in detail. It is shown that while the CLCN method is equivalent to a HO MOM, it offers the advantages of a point based implementation scheme. The numerical results of the application of CLCN to different SIEs including EFIE, MFIE, and AEFIE are demonstrated.

Copyright (C Nastaran Hendijani 2015 


\section{Chapter 2. INTEGRAL EQUATION METHODS}

Surface and volume integral equation methods have been effectively applied to solve various electromagnetic radiation and scattering problems [1, 11]. In this chapter, we review the surface integral equations (SIEs) for perfect electric conductors (PECs) and volume integral equations (VIEs) for penetrable materials.

The main SIEs developed for PECs include electric field integral equation (EFIE), magnetic field integral equation (MFIE), and combined field integral equation (CFIE). Choosing the appropriate method depends on the problem type and the geometrical specifications of the sctterer. As will be discussed in the following, the EFIE is a first kind integral equation; while, the MFIE is a second kind integral equation. As a consequence, the MFIE provides better condition numbers of the system matrix relative to the EFIE. However, the MFIE cannot be applied to scattering problems involving open geometries and demonstrates inferior solution accuracy for objects with singular geometries as compared to the EFIE [35].

One important drawback of the EFIE is its low frequency instability which is a result of the imbalance between the rotational and irrotational parts of the currents at low frequency. Several methods have been invented to address this problem including the augmented EFIE (AEFIE) [31], EFIE-hd [36], self-stabilizing methods [37, 38], and the methods based on the Helmholtz decomposition of the basis functions [39].

For closed geometries, both the EFIE and MFIE show internal resonances in their frequency response [11]. To tackle this problem, CFIE has been devised by linearly combining the EFIE and MFIE. The CFIE demonstrates stable condition numbers at resonance frequencies of the EFIE and MFIE and is useful for solving scattering 
problems involving closed PEC objects. However, the low frequency breakdown of the EFIE is inherited by the CFIE formulation. To remove the low-frequency instability of the CFIE, the CFIE-hd formulation has been developed [40, 41].

For problems where the material properties of the scatterer are spatially changing the VIE formulation is commonly utilized wherein the unknown quantities are distributed through the volume of the scatterer.

\subsection{Maxwell's Equations and Equivalence Principles}

Consider a scatterer specified by the permittivity $\varepsilon_{2}(x, y, z)$ and the permeability $\mu_{2}(x, y, z)$ located in a source free region of a homogenous background material with constitutive parameters $\varepsilon_{1}$ and $\mu_{1}$. The scatterer is illuminated by a time-harmonic electromagnetic wave with time dependence $e^{j \omega t}$. The electric and magnetic fields, $\mathbf{E}$ and $\mathbf{H}$, on the domain of the scatterer are governed by the Maxwell's equations [1]:

$$
\begin{gathered}
\nabla \times \mathbf{E}=-j \omega \mu_{2} \mathbf{H}, \\
\nabla \times \mathbf{H}=j \omega \varepsilon_{2} \mathbf{E}, \\
\nabla \cdot\left(\varepsilon_{2} \mathbf{E}\right)=0, \\
\nabla \cdot\left(\mu_{2} \mathbf{H}\right)=0,
\end{gathered}
$$

The boundary conditions along the interface of the scatterer and the background material are expressed as:

$$
\begin{gathered}
\hat{\mathbf{n}} \times\left(\mathbf{E}_{1}-\mathbf{E}_{2}\right)=0, \\
\hat{\mathbf{n}} \times\left(\mathbf{H}_{1}-\mathbf{H}_{2}\right)=0,
\end{gathered}
$$




$$
\begin{gathered}
\hat{\mathbf{n}} \cdot\left(\varepsilon_{1} \mathbf{E}_{1}-\varepsilon_{2} \mathbf{E}_{2}\right)=0, \\
\hat{\mathbf{n}} \cdot\left(\mu_{1} \mathbf{H}_{1}-\mu_{2} \mathbf{H}_{2}\right)=0,
\end{gathered}
$$

where, $\hat{\mathbf{n}}$ is normal to the boundary surface pointing into the background material. For a PEC scatterer, the following equations are satisfied by the electric and magnetic fields on its boundary:

$$
\begin{gathered}
\hat{\mathbf{n}} \times \mathbf{E}=0, \\
\hat{\mathbf{n}} \times \mathbf{H}=\mathbf{J}_{s}, \\
\hat{\mathbf{n}} \cdot \mathbf{E}=\rho_{s} / \varepsilon_{1}, \\
\hat{\mathbf{n}} \cdot \mathbf{H}=0,
\end{gathered}
$$

$\mathbf{J}_{s}$ and $\rho_{s}$ are respectively the surface current and charge densities. The vector Helmholtz equations are derived by combining (2.1) and (2.2) as:

$$
\begin{aligned}
& \nabla \times\left(\frac{\mu_{1}}{\mu_{2}} \nabla \times \mathbf{E}\right)-k_{1}^{2} \frac{\varepsilon_{2}}{\varepsilon_{1}} \mathbf{E}=0 \\
& \nabla \times\left(\frac{\varepsilon_{1}}{\varepsilon_{2}} \nabla \times \mathbf{H}\right)-k_{1}^{2} \frac{\mu_{2}}{\mu_{1}} \mathbf{H}=0
\end{aligned}
$$

where, $k_{1}^{2}=\omega^{2} \mu_{1} \varepsilon_{1} . k_{1}$ is the wave number of the background medium.

As an alternative to PDEs provided by (2.13) and (2.14), integral equations are commonly applied to analyze the electromagnetic scattering. It is not trivial to obtain the integral equations directly from (2.1) to (2.4), since the electric and magnetic fields are radiating in a mixed region including different materials. As a result, the inhomogeneous scatterer is replaced by the equivalent volume currents and charges radiating in the background material. The equivalent problem is formulated as: 


$$
\begin{gathered}
\nabla \times \mathbf{E}=-j \omega \mu_{1} \mathbf{H}-\mathbf{M}_{v}, \\
\nabla \times \mathbf{H}=j \omega \varepsilon_{1} \mathbf{E}+\mathbf{J}_{v}, \\
\nabla \cdot\left(\varepsilon_{1} \mathbf{E}\right)=\rho_{v e}, \\
\nabla \cdot\left(\mu_{1} \mathbf{H}\right)=\rho_{v m},
\end{gathered}
$$

where, $\mathbf{J}$ and $\mathbf{M}$ are respectively equivalent volume electric and magnetic current densities. $\rho_{v e}$ and $\rho_{v m}$ are equivalent electric and magnetic charge densities. The equivalent sources are defined as:

$$
\begin{gathered}
\mathbf{M}_{v}=j \omega\left(\mu_{2}-\mu_{1}\right) \mathbf{H}, \\
\mathbf{J}_{v}=j \omega\left(\varepsilon_{2}-\varepsilon_{1}\right) \mathbf{E}, \\
\rho_{v e}=\varepsilon_{2} \mathbf{E} \cdot \nabla\left(\frac{\varepsilon_{1}}{\varepsilon_{2}}\right), \\
\rho_{v m}=\mu_{2} \mathbf{H} \cdot \nabla\left(\frac{\mu_{1}}{\mu_{2}}\right) .
\end{gathered}
$$

The equations (2.15) to (2.18) describe the volume equivalence principle. Therefore, the original problem including different materials is reduced to a number of equivalent sources radiating in the background material. However, the sources in (2.19) to (2.22) are also unknown and should be computed through solving the integral equations which will be discussed later in this chapter.

For scattering from homogenous objects the surface equivalence principle provides a more computationally efficient formulation compared to the volume equivalence approach. The reason is that the unknown source quantities will be confined to the surface of the scattering object. The surface equivalence principle is a consequence of the 
Lorentz reciprocity theorem [1] and is depicted in Figure 1. The electromagnetic fields outside the boundaries of the homogenous scatterer with material properties $\varepsilon_{2}$ and $\mu_{2}$ embedded in the background material $\varepsilon_{1}$ and $\mu_{1}$ are equivalent to the fields generated by the surface electric and magnetic current sources:

$$
\begin{aligned}
\mathbf{J}_{s} & =\hat{\mathbf{n}} \times \mathbf{H}_{1}, \\
\mathbf{M}_{s} & =\mathbf{E}_{1} \times \hat{\mathbf{n}},
\end{aligned}
$$

positioned on the surface of the object and radiating in background material. $\hat{\mathbf{n}}$ is the normal vector pointing outwards the object. This is called the exterior surface equivalence principle. Similarly, the fields interior to the objects are the solution of the equivalent problem including the surface current sources:

$$
\begin{array}{r}
\mathbf{J}_{s}=-\hat{\mathbf{n}} \times \mathbf{H}_{2}, \\
\mathbf{M}_{s}=-\mathbf{E}_{2} \times \hat{\mathbf{n}},
\end{array}
$$

placed on the surface of the object radiating in the homogenous material with constitutive parameters $\varepsilon_{2}$ and $\mu_{2}$.

To demonstrate the equivalence principle for a PEC scatterer, we note that on the surface of a PEC the tangential component of the electric field is zero according to the boundary condition (2.9). By utilizing (2.23) and (2.24) the fields in the region outside the PEC scatterer are generated by the equivalent sources:

$$
\begin{gathered}
\mathbf{J}_{s}=\hat{\mathbf{n}} \times \mathbf{H}, \\
\mathbf{M}_{s}=0,
\end{gathered}
$$

located on the surface of the PEC object. 


\subsection{Formulating the Scattering Problem}

Assume a scatterer with constitutive parameters $\left(\varepsilon_{2}, \mu_{2}\right)$ located in the background

material $\left(\varepsilon_{1}, \mu_{1}\right)$ is illuminated by the incident fields $\mathbf{E}^{\text {inc }}$ and $\mathbf{H}^{\text {inc }}$ produced by a source outside the scatterer. As discussed in the previous section, the scatterer can be replaced by the equivalent currents using either the surface or volume equivalence principle. As a result, the fields outside the scatterer are a superposition of the original incident fields and the fields generated by the induced equivalent currents [1]:

$$
\begin{array}{r}
\mathbf{E}=\mathbf{E}^{i n c}+\mathbf{E}^{s}, \\
\mathbf{H}=\mathbf{H}^{i n c}+\mathbf{H}^{s},
\end{array}
$$

where, $\mathbf{E}$ and $\mathbf{H}$ are respectively the total electric and magnetic fields outside the scatterer. $\mathbf{E}^{s}$ and $\mathbf{H}^{s}$ are the scattered fields produced by the equivalent currents. The scattered fields satisfy the following equations:

$$
\begin{gathered}
\nabla^{2} \mathbf{E}^{s}-k_{1}^{2} \mathbf{E}^{s}=j \omega \mu_{1} \mathbf{J}-\frac{\nabla \nabla \cdot \mathbf{J}}{j \omega \varepsilon_{1}}+\nabla \times \mathbf{M}, \\
\nabla^{2} \mathbf{H}^{s}-k_{1}^{2} \mathbf{H}^{s}=-\nabla \times \mathbf{J}+j \omega \varepsilon_{1} \mathbf{M}-\frac{\nabla \nabla \cdot \mathbf{M}}{j \omega \mu_{1}},
\end{gathered}
$$

where $\mathbf{J}$ and $\mathbf{M}$ are either volume or surface equivalent currents, and $k_{1}$ is the wave number of the background medium. It should be noted that the equivalent currents are a function of the total fields, $\mathbf{E}$ and $\mathbf{H}$. The radiation conditions are applied to uniquely define the solution of the equations (2.31) and (2.32).

$$
\begin{aligned}
& \lim _{r \rightarrow \infty} \hat{\mathbf{r}} \times \nabla \times \mathbf{E}^{s}=j k_{1} \mathbf{E}^{s} \\
& \lim _{r \rightarrow \infty} \hat{\mathbf{r}} \times \nabla \times \mathbf{H}^{s}=j k_{1} \mathbf{H}^{s}
\end{aligned}
$$


where, $r$ is in the spherical coordinate. In fact the conditions (2.33) and (2.34) guarantee that the fields propagate outwards relative to the scatterer.

Several approaches have been sought to solve (2.31) and (2.32) in order to find the relationship between the scattered fields and the equivalent sources. One way is to define the scattered fields in terms of vector magnetic potential $\mathbf{A}$ and the vector electric potential $\mathbf{F}$ :

$$
\begin{aligned}
\mathbf{E}^{s} & =\frac{\nabla \nabla \cdot \mathbf{A}+k_{1}^{2} \mathbf{A}}{j \omega \varepsilon_{1}}-\nabla \times \mathbf{F} . \\
\mathbf{H}^{s} & =\nabla \times \mathbf{A}+\frac{\nabla \nabla \cdot \mathbf{F}+k_{1}^{2} \mathbf{F}}{j \omega \mu_{1}} .
\end{aligned}
$$

Substituting (2.35) and (2.36) into the Maxwell's equations, we obtain:

$$
\begin{aligned}
& \nabla^{2} \mathbf{A}+k_{1}^{2} \mathbf{A}=-\mathbf{J} . \\
& \nabla^{2} \mathbf{F}+k_{1}^{2} \mathbf{F}=-\mathbf{M} .
\end{aligned}
$$

The solutions to these equations which also satisfy the radiation conditions (2.33) and (2.34) can be expressed as:

$$
\begin{gathered}
\mathbf{A}(\mathbf{r})=\int_{\Omega^{\prime}} \mathbf{J}\left(\mathbf{r}^{\prime}\right) G\left(\mathbf{r}, \mathbf{r}^{\prime}\right) d \Omega^{\prime} . \\
\mathbf{F}(\mathbf{r})=\int_{\Omega^{\prime}} \mathbf{M}\left(\mathbf{r}^{\prime}\right) G\left(\mathbf{r}, \mathbf{r}^{\prime}\right) d \Omega^{\prime} .
\end{gathered}
$$

where, $\Omega^{\prime}$ is the domain of the equivalent current sources, and $G$ is the scalar Green's function:

$$
G\left(\mathbf{r}, \mathbf{r}^{\prime}\right)=\frac{\exp \left(-j k_{1}\left|\mathbf{r}-\mathbf{r}^{\prime}\right|\right)}{4 \pi\left|\mathbf{r}-\mathbf{r}^{\prime}\right|}
$$


Utilizing (2.39) and (2.40) in conjunction with (2.35) and (2.36), the scattered fields can be demonstrated as:

$$
\begin{aligned}
\mathbf{E}^{s}= & -j \omega \mu_{1} \int_{\Omega^{\prime}} \mathbf{J}\left(\mathbf{r}^{\prime}\right) G\left(\mathbf{r}, \mathbf{r}^{\prime}\right) d \Omega^{\prime}+\frac{1}{j \omega \varepsilon_{1}} \nabla \nabla \cdot \int_{\Omega^{\prime}} \mathbf{J}\left(\mathbf{r}^{\prime}\right) G\left(\mathbf{r}, \mathbf{r}^{\prime}\right) d \Omega^{\prime} \\
& -\int_{\Omega^{\prime}} \nabla \times \mathbf{M}\left(\mathbf{r}^{\prime}\right) G\left(\mathbf{r}, \mathbf{r}^{\prime}\right) d \Omega^{\prime} . \\
\mathbf{H}^{s}= & \int_{\Omega^{\prime}} \nabla \times \mathbf{J}\left(\mathbf{r}^{\prime}\right) G\left(\mathbf{r}, \mathbf{r}^{\prime}\right) d \Omega^{\prime}-j \omega \varepsilon_{1} \int_{\Omega^{\prime}} \mathbf{M}\left(\mathbf{r}^{\prime}\right) G\left(\mathbf{r}, \mathbf{r}^{\prime}\right) d \Omega^{\prime} \\
& +\frac{1}{j \omega \mu_{1}} \nabla \nabla \cdot \int_{\Omega^{\prime}} \mathbf{M}\left(\mathbf{r}^{\prime}\right) G\left(\mathbf{r}, \mathbf{r}^{\prime}\right) d \Omega^{\prime} .
\end{aligned}
$$

Now that the scattered fields have been obtained in terms of equivalent sources, the surface integral equations are constructed by combining (2.29) and (2.30) with the equivalence principles. The electric field integral equation (EFIE) is a result of enforcing the tangential component of the electric field to zero on the surface of the PEC scatterer:

$$
\hat{\mathbf{n}} \times \mathbf{E}=0
$$

Substituting (2.29) in (2.44):

$$
\hat{\mathbf{n}} \times\left(\mathbf{E}^{i n c}+\mathbf{E}^{s}\right)=0
$$

Utilizing (2.42) and noting that there is no magnetic equivalent current on the surface of the PEC scatterer, (2.45) is represented as:

$$
-\hat{\mathbf{n}} \times \mathbf{E}^{i n c}=-j \omega \mu_{1} \hat{\mathbf{n}} \times \int_{S} \mathbf{J}_{s}\left(\mathbf{r}^{\prime}\right) G\left(\mathbf{r}, \mathbf{r}^{\prime}\right) d s^{\prime}+\hat{\mathbf{n}} \frac{1}{j \omega \varepsilon_{1}} \times \nabla \nabla \cdot \int_{S} \mathbf{J}_{s}\left(\mathbf{r}^{\prime}\right) G\left(\mathbf{r}, \mathbf{r}^{\prime}\right) d s^{\prime}
$$

The scalar expression for EFIE can be achieved via carrying out the vector product of $-\mathbf{t} \cdot \hat{\mathbf{n}} \times$ (where $\mathbf{t}$ is a vector tangential to the surface of the scatterer) with both sides of (2.46) and using the vector identity $\mathbf{a} \times(\mathbf{b} \times \mathbf{c})=(\mathbf{a} \cdot \mathbf{c}) \mathbf{b}-(\mathbf{a} \cdot \mathbf{b}) \mathbf{c}$ : 


$$
-\mathbf{t} \cdot \mathbf{E}^{i n c}(\mathbf{r})=-j k_{1} \eta_{1} \mathbf{t} \cdot \int_{S} \mathbf{J}_{s}\left(\mathbf{r}^{\prime}\right) G\left(\mathbf{r}, \mathbf{r}^{\prime}\right) d s^{\prime}-j \frac{\eta_{1}}{k_{1}} \mathbf{t} \cdot \nabla \nabla \cdot \int_{S} \mathbf{J}_{s}\left(\mathbf{r}^{\prime}\right) G\left(\mathbf{r}, \mathbf{r}^{\prime}\right) d s^{\prime}
$$

where, $\eta_{1}=\sqrt{\mu_{1} / \varepsilon_{1}}$ is the characteristic impedance of the background medium. Equation (2.47) can be rewritten as:

$$
-\mathbf{t} \cdot \mathbf{E}^{i n c}(\mathbf{r})=\mathbf{t} \cdot \int_{S} \overline{\mathbf{G}}_{e}\left(\mathbf{r}, \mathbf{r}^{\prime}\right) \cdot \mathbf{J}_{s}\left(\mathbf{r}^{\prime}\right) d s^{\prime}
$$

where $\overline{\mathbf{G}}_{e}\left(\mathbf{r}, \mathbf{r}^{\prime}\right)$ is the electric dyadic Green's function defined as:

$$
\overline{\mathbf{G}}_{e}\left(\mathbf{r}, \mathbf{r}^{\prime}\right)=-j k_{1} \eta_{1}\left[\overline{\mathbf{I}}+\frac{1}{k_{1}^{2}} \nabla \nabla\right] G\left(\mathbf{r}, \mathbf{r}^{\prime}\right),
$$

and $\overline{\mathbf{I}}$ is the unit dyad. The MFIE is derived by combining (2.30) and (2.27):

$$
\hat{\mathbf{n}} \times\left.\left(\mathbf{H}^{i n c}+\mathbf{H}^{s}\right)\right|_{S^{+}}=\mathbf{J}_{s},
$$

where, $S^{+}$is the surface in the vicinity of the scatterer in the background medium. Applying (2.43) and considering the fact that there is no magnetic equivalent current on the surface of the PEC scatterer, MFIE is demonstrated as:

$$
\hat{\mathbf{n}} \times \mathbf{H}^{i n c}(\mathbf{r})=\mathbf{J}_{s}-\hat{\mathbf{n}} \times \nabla \times \int_{S} \mathbf{J}_{s}\left(\mathbf{r}^{\prime}\right) G\left(\mathbf{r}, \mathbf{r}^{\prime}\right) d s^{\prime},
$$

Since, $\mathbf{H}^{s}$ is discontinuous on the surface of the PEC scatterer, the integral in (2.51) is a principal value integral. Calculating the principal value integral, (2.51) is represented as [35]:

$$
\hat{\mathbf{n}} \times \mathbf{H}^{i n c}(\mathbf{r})=\frac{1}{2} \mathbf{J}_{s}-\hat{\mathbf{n}} \times \nabla \times \int_{S} \mathbf{J}_{s}\left(\mathbf{r}^{\prime}\right) G\left(\mathbf{r}, \mathbf{r}^{\prime}\right) d s^{\prime},
$$

where, $f_{s}$ is the principal value integral. Manipulating (2.52) by applying the vector identities, we have: 


$$
\hat{\mathbf{n}} \times \mathbf{H}^{i n c}(\mathbf{r})=\frac{1}{2} \mathbf{J}_{s}-\hat{\mathbf{n}} \times f_{S} \nabla G\left(\mathbf{r}, \mathbf{r}^{\prime}\right) \times \mathbf{J}_{s}\left(\mathbf{r}^{\prime}\right) d s^{\prime} .
$$

The scalar form of MFIE can be obtained by the application of the dot product of the vector $\mathbf{t}$ (tangential to the surface of the PEC scatterer) to the both sides of (2.52):

$$
\mathbf{t} \cdot \hat{\mathbf{n}} \times \mathbf{H}^{i n c}(\mathbf{r})=\frac{1}{2} \mathbf{t} \cdot \mathbf{J}_{s}-\mathbf{t} \cdot \hat{\mathbf{n}} \times \oint_{S} \nabla G\left(\mathbf{r}, \mathbf{r}^{\prime}\right) \times \mathbf{J}_{s}\left(\mathbf{r}^{\prime}\right) d s^{\prime}
$$

The final form of MFIE is expressed as:

$$
\mathbf{t} \cdot \hat{\mathbf{n}} \times \mathbf{H}^{i n c}(\mathbf{r})=\frac{1}{2} \mathbf{t} \cdot \mathbf{J}_{s}-(\mathbf{t} \times \hat{\mathbf{n}}) \cdot f_{S} \nabla G\left(\mathbf{r}, \mathbf{r}^{\prime}\right) \times \mathbf{J}_{s}\left(\mathbf{r}^{\prime}\right) d s^{\prime}
$$

The CFIE formulation is defined as the linear combination of EFIE and MFIE in (2.47) and (2.55):

$$
\begin{aligned}
\alpha \mathbf{t} \cdot \mathbf{E}^{i n c}(\mathbf{r})+(1-\alpha) \eta_{1} \mathbf{H}^{i n c}(\mathbf{r}) & =j \alpha k_{1} \eta_{1} \mathbf{t} \cdot \int_{s} \mathbf{J}_{s}\left(\mathbf{r}^{\prime}\right) G\left(\mathbf{r}, \mathbf{r}^{\prime}\right) d s^{\prime} \\
& +j \alpha \frac{\eta_{1}}{k_{1}} \mathbf{t} \cdot \nabla \nabla \cdot \int_{s} \mathbf{J}_{s}\left(\mathbf{r}^{\prime}\right) G\left(\mathbf{r}, \mathbf{r}^{\prime}\right) d s^{\prime}+\frac{1}{2}(1-\alpha) \eta_{1} \mathbf{t} \cdot \mathbf{J}_{s} \\
& -(1-\alpha) \eta_{1}(\mathbf{t} \times \hat{\mathbf{n}}) \cdot f_{S} \nabla G\left(\mathbf{r}, \mathbf{r}^{\prime}\right) \times \mathbf{J}_{s}\left(\mathbf{r}^{\prime}\right) d s^{\prime}
\end{aligned}
$$

where $0 \leq \alpha \leq 1$. As it was mentioned before unlike the EFIE and MFIE, the frequency response of the CFIE is resonance free.

In order to derive the volume integral equations, we consider the combination of (2.19), (2.20), (2.29), and (2.30):

$$
\begin{gathered}
\mathbf{E}^{i n c}+\mathbf{E}^{s}=\frac{\mathbf{J}_{v}}{j \omega\left(\varepsilon_{2}-\varepsilon_{1}\right)}, \\
\mathbf{H}^{i n c}+\mathbf{H}^{s}=\frac{\mathbf{M}_{v}}{j \omega\left(\mu_{2}-\mu_{1}\right)} .
\end{gathered}
$$


Substituting (2.42) and (2.43) in the above equations, the volume electric field integral equation (VEFIE) and magnetic field integral equation (VMFIE) are respectively defined as:

$$
\begin{aligned}
\mathbf{E}^{i n c}(\mathbf{r}) & =\frac{\mathbf{J}_{v}(\mathbf{r})}{j \omega\left(\varepsilon_{2}(\mathbf{r})-\varepsilon_{1}\right)}+j k_{1} \eta_{1} \int_{V} \mathbf{J}_{v}\left(\mathbf{r}^{\prime}\right) G\left(\mathbf{r}, \mathbf{r}^{\prime}\right) d v^{\prime} \\
& +j k_{1}^{-1} \eta_{1} \nabla \nabla \cdot \int_{V} \mathbf{J}_{v}\left(\mathbf{r}^{\prime}\right) G\left(\mathbf{r}, \mathbf{r}^{\prime}\right) d v^{\prime}+\int_{V} \nabla G\left(\mathbf{r}, \mathbf{r}^{\prime}\right) \times \mathbf{M}_{v}\left(\mathbf{r}^{\prime}\right) d v^{\prime} \\
\mathbf{H}^{i n c}(\mathbf{r}) & =\frac{\mathbf{M}_{v}(\mathbf{r})}{j \omega\left(\mu_{2}(\mathbf{r})-\mu_{1}\right)}+j k_{1} \eta_{1}^{-1} \int_{V} \mathbf{M}_{v}\left(\mathbf{r}^{\prime}\right) G\left(\mathbf{r}, \mathbf{r}^{\prime}\right) d v^{\prime} \\
& +j k_{1}^{-1} \eta_{1}^{-1} \nabla \nabla \cdot \int_{V} \mathbf{M}_{v}\left(\mathbf{r}^{\prime}\right) G\left(\mathbf{r}, \mathbf{r}^{\prime}\right) d v^{\prime}-\int_{V} \nabla G\left(\mathbf{r}, \mathbf{r}^{\prime}\right) \times \mathbf{J}_{v}\left(\mathbf{r}^{\prime}\right) d v^{\prime} .
\end{aligned}
$$

where, $\mathbf{r} \in V$. By choosing an appropriate test vector $\mathbf{t}$, the scalar form of vector equations (2.59) and (2.60) are derived:

$$
\begin{aligned}
\mathbf{t} \cdot \mathbf{E}^{i n c}(\mathbf{r}) & =\frac{\mathbf{t} \cdot \mathbf{J}_{v}(\mathbf{r})}{j \omega\left(\varepsilon_{2}(\mathbf{r})-\varepsilon_{1}\right)}+j k_{1} \eta_{1} \mathbf{t} \cdot \int_{V} \mathbf{J}_{v}\left(\mathbf{r}^{\prime}\right) G\left(\mathbf{r}, \mathbf{r}^{\prime}\right) d v^{\prime} \\
& +j k_{1}^{-1} \eta_{1} \mathbf{t} \cdot \nabla \nabla \cdot \int_{V} \mathbf{J}_{v}\left(\mathbf{r}^{\prime}\right) G\left(\mathbf{r}, \mathbf{r}^{\prime}\right) d v^{\prime}+\mathbf{t} \cdot \int_{V} \nabla G\left(\mathbf{r}, \mathbf{r}^{\prime}\right) \times \mathbf{M}_{v}\left(\mathbf{r}^{\prime}\right) d v^{\prime} \\
\mathbf{t} \cdot \mathbf{H}^{i n c}(\mathbf{r}) & =\frac{\mathbf{t} \cdot \mathbf{M}_{v}(\mathbf{r})}{j \omega\left(\mu_{2}(\mathbf{r})-\mu_{1}\right)}+j k_{1} \eta_{1}^{-1} \mathbf{t} \cdot \int_{V} \mathbf{M}_{v}\left(\mathbf{r}^{\prime}\right) G\left(\mathbf{r}, \mathbf{r}^{\prime}\right) d v^{\prime} \\
& +j k_{1}^{-1} \eta_{1}^{-1} \mathbf{t} \cdot \nabla \nabla \cdot \int_{V} \mathbf{M}_{v}\left(\mathbf{r}^{\prime}\right) G\left(\mathbf{r}, \mathbf{r}^{\prime}\right) d v^{\prime}-\mathbf{t} \cdot \int_{V} \nabla G\left(\mathbf{r}, \mathbf{r}^{\prime}\right) \times \mathbf{J}_{v}\left(\mathbf{r}^{\prime}\right) d v^{\prime}
\end{aligned}
$$

For a purely dielectric inhomogeneous material, only the equivalent volume electric currents are defined, and just the VEFIE is applied to solve for the unknown current densities:

$$
\begin{aligned}
\mathbf{t} \cdot \mathbf{E}^{i n c}(\mathbf{r}) & =\frac{\mathbf{t} \cdot \mathbf{J}_{v}(\mathbf{r})}{j \omega\left(\varepsilon_{2}(\mathbf{r})-\varepsilon_{1}\right)}+j k_{1} \eta_{1} \mathbf{t} \cdot \int_{V} \mathbf{J}_{v}\left(\mathbf{r}^{\prime}\right) G\left(\mathbf{r}, \mathbf{r}^{\prime}\right) d v^{\prime} \\
& +j k_{1}^{-1} \eta_{1} \mathbf{t} \cdot \nabla \nabla \cdot \int_{V} \mathbf{J}_{v}\left(\mathbf{r}^{\prime}\right) G\left(\mathbf{r}, \mathbf{r}^{\prime}\right) d v^{\prime}
\end{aligned}
$$


It is noticed that the VMFIE is not solvable in this case. In a similar way, for a purely magnetic material only the equivalent volume magnetic currents exist, and the VMFIE is applied to describe the scattering problem:

$$
\begin{aligned}
\mathbf{t} \cdot \mathbf{H}^{i n c}(\mathbf{r}) & =\frac{\mathbf{t} \cdot \mathbf{M}_{v}(\mathbf{r})}{j \omega\left(\mu_{2}(\mathbf{r})-\mu_{1}\right)}+j k_{1} \eta_{1}^{-1} \mathbf{t} \cdot \int_{V} \mathbf{M}_{v}\left(\mathbf{r}^{\prime}\right) G\left(\mathbf{r}, \mathbf{r}^{\prime}\right) d v^{\prime} \\
& +j k_{1}^{-1} \eta_{1}^{-1} \mathbf{t} \cdot \nabla \nabla \cdot \int_{V} \mathbf{M}_{v}\left(\mathbf{r}^{\prime}\right) G\left(\mathbf{r}, \mathbf{r}^{\prime}\right) d v^{\prime}
\end{aligned}
$$

\section{3. $\mathrm{L}$ and $\mathrm{K}$ Operators}

Considering the derived surface and volume integral equations in the previous section, we find an analogy between different terms of the equations. Consequently, by defining the $\mathbf{L}$ and $\mathbf{K}$ operators:

$$
\begin{gathered}
\mathbf{L}(X)=\int_{\Omega}-j k_{1}\left[\overline{\mathbf{I}}+\frac{1}{k_{1}^{2}} \nabla \nabla\right] G\left(\mathbf{r}, \mathbf{r}^{\prime}\right) \cdot X\left(\mathbf{r}^{\prime}\right) d \Omega^{\prime}, \\
\mathbf{K}(X)=\int_{\Omega} \nabla G\left(\mathbf{r}, \mathbf{r}^{\prime}\right) \times X\left(\mathbf{r}^{\prime}\right) d \Omega^{\prime},
\end{gathered}
$$

where, $\Omega$ is the domain of $X$. The EFIE formulation is expressed as:

$$
\mathbf{t} \cdot \mathbf{E}^{\text {inc }}(\mathbf{r})=-\eta_{1} \mathbf{t} \cdot \mathbf{L}\left(\mathbf{J}_{s}\right)
$$

and the MFIE is expressed as:

$$
\mathbf{t} \cdot \hat{\mathbf{n}} \times \mathbf{H}^{i n c}(\mathbf{r})=\frac{1}{2} \mathbf{t} \cdot \mathbf{J}_{s}-(\mathbf{t} \times \hat{\mathbf{n}}) \cdot \overline{\mathbf{K}}\left(\mathbf{J}_{s}\right) .
$$

The VIE formulations are then represented as:

$$
\begin{gathered}
\mathbf{t} \cdot \mathbf{E}^{i n c}(\mathbf{r})=\frac{\mathbf{t} \cdot \mathbf{J}_{v}(\mathbf{r})}{j \omega\left(\varepsilon_{2}(\mathbf{r})-\varepsilon_{1}\right)}-\eta_{1} \mathbf{t} \cdot \mathbf{L}\left(\mathbf{J}_{v}\right)+\mathbf{t} \cdot \overline{\mathbf{K}}\left(\mathbf{M}_{v}\right) . \\
\mathbf{t} \cdot \mathbf{H}^{i n c}(\mathbf{r})=\frac{\mathbf{t} \cdot \mathbf{M}_{v}(\mathbf{r})}{j \omega\left(\mu_{2}(\mathbf{r})-\mu_{1}\right)}-\eta_{1}^{-1} \mathbf{t} \cdot \overline{\mathbf{L}}\left(\mathbf{M}_{v}\right)-\mathbf{t} \cdot \overline{\mathbf{K}}\left(\mathbf{J}_{v}\right) .
\end{gathered}
$$


The above representations are useful in explaining the LCN discretization of the integral equations in the next chapter.

\subsection{The Frequency Instability of the EFIE}

As it was mentioned before, one limitation of the EFIE is that it breaks down at low frequency. This occurs because of the imbalance of the contribution from the rotational and irrotational parts of currents to the $\mathbf{L}$ operator at low frequency $[42,43]$. As a consequence, the EFIE system matrix becomes ill-conditioned at low frequency. To elucidate the low frequency failure of the EFIE, we start from (2.47). By applying the vector identities, (2.47) can be expressed as [31]:

$$
\mathbf{t} \cdot \mathbf{E}^{i n c}(\mathbf{r})=j k_{1} \eta_{1} \mathbf{t} \cdot \int_{S} \mathbf{J}_{s}\left(\mathbf{r}^{\prime}\right) G\left(\mathbf{r}, \mathbf{r}^{\prime}\right) d s^{\prime}+j \frac{\eta_{1}}{k_{1}} \mathbf{t} \cdot \nabla \int_{S} G\left(\mathbf{r}, \mathbf{r}^{\prime}\right) \nabla^{\prime} \cdot \mathbf{J}_{s}\left(\mathbf{r}^{\prime}\right) d s^{\prime}
$$

The surface current $\mathbf{J}_{s}$ is represented as sum of its rotational and irrotational components:

$$
\mathbf{J}_{s}=\mathbf{J}_{s}^{R}+\mathbf{J}_{s}^{I}
$$

The divergence of the rotational part of the current is equal to zero:

$$
\nabla \cdot \mathbf{J}_{s}^{R}=0
$$

Consequently, as the frequency tends to zero (2.71) can be represented as:

$$
\lim _{\omega \rightarrow 0}\left(\mathbf{t} \cdot \mathbf{E}^{i n c}(\mathbf{r})\right)=\frac{j}{\varepsilon_{1}} \lim _{\omega \rightarrow 0}\left(\mathbf{t} \cdot \nabla \int_{S} G\left(\mathbf{r}, \mathbf{r}^{\prime}\right) \frac{\nabla^{\prime} \cdot \mathbf{J}_{s}^{I}\left(\mathbf{r}^{\prime}\right)}{\omega} d s^{\prime}\right)
$$

Accordingly, at low frequency the electric field computed based on the EFIE depends only on the irotational part of the current. Since, there is no dependency on the rotational part of the current, the system becomes under-constrained and unstable. Equation (2.74) 
implicitly indicates that the irrotational part of the current is proportional to $\omega$, i.e. $\mathbf{J}_{s}^{I} \sim O(\omega)$. The fact that the irrotaional part of the current is proportional to $\omega$ can also be concluded from the current continuity equation [35]:

$$
\nabla \cdot \mathbf{J}_{s}=-j \omega \rho_{e}
$$

Since the rotational part of the current is divergence free, (2.75) is rewritten as:

$$
\rho_{e}=-\frac{\nabla \cdot \mathbf{J}_{s}^{I}}{j \omega}
$$

As a result, as the frequency goes to zero, $\mathbf{J}_{s}^{I}$ must be proportional to $\omega$ to have a finite static charge. The idea of decomposing the current into its rotational and irrotational parts and scaling the irrotational part of the current by $\omega$ have been applied to develop the EFIE-hd formulation which provides stability and well-conditioning at low frequencies [36]. 


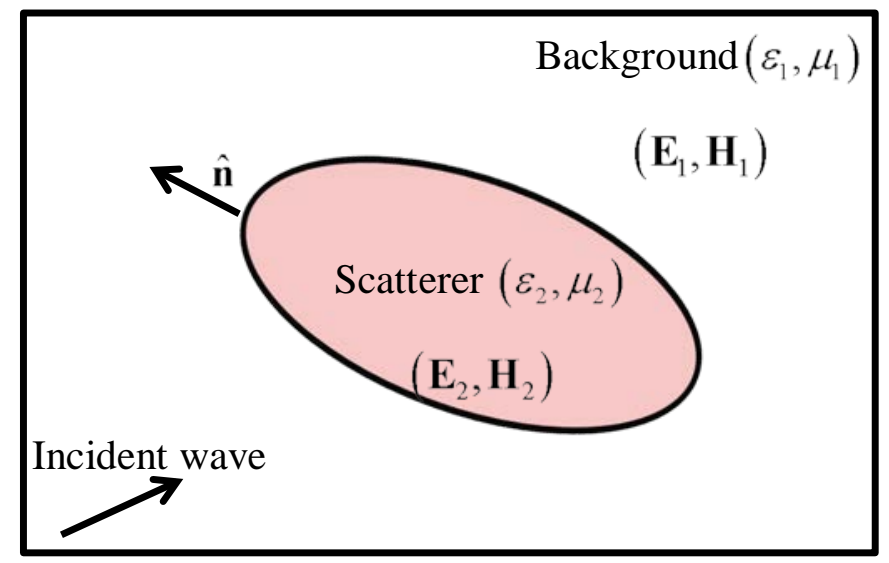

(a)

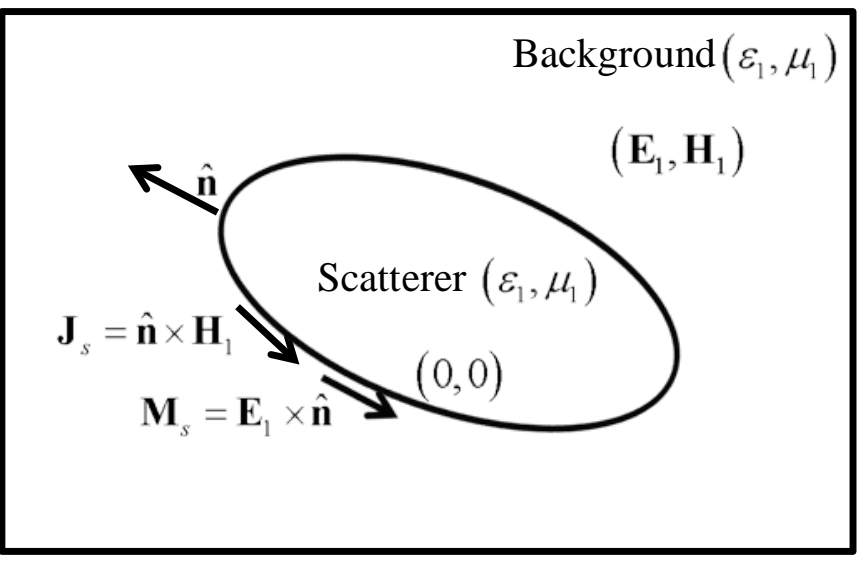

(b)

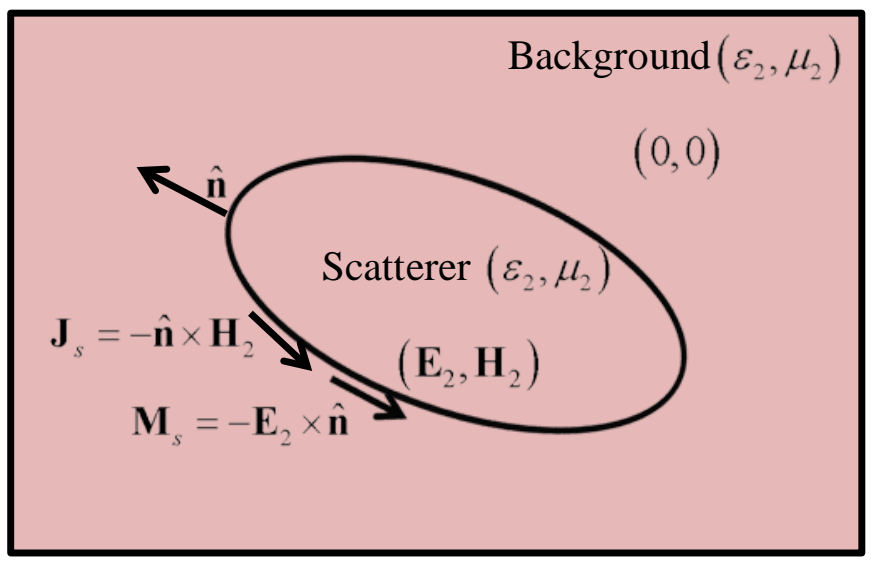

(c)

Figure 1. Surface equivalence principle (a) original problem (b) exterior equivalent problem (c) interior equivalent problem

Copyright (C) Nastaran Hendijani 2015 


\section{Chapter 3. THE LOCALLY CORRECTED NySTRÖM METHOD}

The basic idea of the conventional Nyström method is to approximate the integration in the integral equation by an appropriate quadrature rule and sampling the unknown function at the quadrature points corresponding to the specified quadrature rule. The basic Nyström method demonstrates exponential convergence for smooth integrands by increasing the order of the quadrature rule [44, 45].

However, the integral equations encountered in computational electromagnetics commonly have singular integrands which cannot be treated by regular integration quadrature rules. As a consequence, local corrections were introduced to compute the integrations near the kernel singularity [22]. The HO locally corrected Nyström (LCN) method has been utilized successfully to discretize integral equations [23]. Compared to the method of moments (MoM), the LCN method offers more computational efficiency by circumventing the double integrations through its point based scheme. Moreover, achieving the $\mathrm{HO}$ convergence through a MOM structure requires the construction of $\mathrm{HO}$ basis functions that produces much more complexity compared to the point based scheme of the LCN method. In addition, since the underlying LCN basis functions are confined to individual cells, the LCN approach can be applied in combination with non-conformal meshes. Furthermore, the LCN method offers more flexibility to develop fast direct or iterative solvers $[5,7,17]$.

\subsection{The Original Nyström Method}

To illustrate the Nyström method, we consider a scalar Ferdholm's integral equation of the first kind [45]: 


$$
f(\mathbf{r})=\int_{\Omega} K\left(\mathbf{r}, \mathbf{r}^{\prime}\right) J\left(\mathbf{r}^{\prime}\right) d \Omega^{\prime}
$$

where $K\left(\mathbf{r}, \mathbf{r}^{\prime}\right)$ is a smooth kernel over the domain $\Omega$, and $J$ is the unknown scalar function. $f$ is a known function on the domain $\Omega$. Equation (3.1) can describe a scattering problem with hypothetical scalar currents $J\left(\mathbf{r}^{\prime}\right)$. We assume that the domain $\Omega$ is divided to $N_{s}$ subdomains $\Omega_{n}, n=1, \ldots, N_{s}$, so that the integration in (3.1) on $\Omega_{n}$ can be approximated by an appropriate quadrature rule to a controllable accuracy. Therefore, by applying the quadrature rule integration on the $N_{s}$ subdomains (3.1) is represented as:

$$
f(\mathbf{r}) \approx \sum_{n=1}^{N_{s}} \sum_{q=1}^{N_{q}} \omega_{q_{n}} K\left(\mathbf{r}, \mathbf{r}_{q_{n}}\right) J\left(\mathbf{r}_{q_{n}}\right) \sqrt{g_{q_{n}}}
$$

where, $\mathbf{r}_{q_{n}}$ are the abscissa corresponding to the specified quadrature rule, and $\omega_{q_{n}}$ are the quadrature weights. $N_{q}$ is the number of quadrature points on each subdomain. $\sqrt{g_{q_{n}}}$ is the surface Jacobian computed at $\mathbf{r}_{q_{n}}$ [11]. For non-singular kernels $K\left(\mathbf{r}, \mathbf{r}^{\prime}\right)$ the error of the integration approximation given by (3.2) converges exponentially by increasing the order of the quadrature rule. The basic idea of the Nyström method is to find the samples of the unknown function at the discrete points corresponding to the abscissa of the quadrature rule. This is unlike the MOM in which the unknowns are the coefficients of the basis functions applied to obtain the expansion of the unknown function. To obtain $N$ unknown samples of $J$ where $N=N_{q} N_{s}$, a linear system of equations is set up by testing (3.2) at the $N$ quadrature points:

$$
f\left(\mathbf{r}_{q_{m}}\right)=\sum_{n=1}^{N_{s}} \sum_{q=1}^{N_{q}} \omega_{q_{n}} K\left(\mathbf{r}_{q_{m}}, \mathbf{r}_{q_{n}}\right) J\left(\mathbf{r}_{q_{n}}\right) \sqrt{g_{q_{n}}},
$$


where, $\mathbf{r}_{q_{m}}$ are the quadrature abscissa on $\Omega_{m}$. Equation (3.3) results in a matrix equation:

$$
[Z] \overline{\mathrm{j}}=\overline{\mathrm{f}}
$$

where, $[Z]$ is an $N \times N$ matrix with entries $\omega_{q_{n}} K\left(\mathbf{r}_{q_{m}}, \mathbf{r}_{q_{n}}\right) \sqrt{g_{q_{n}}} \cdot \overline{\mathrm{j}}$ and $\overline{\mathrm{f}}$ are column vectors (indicated by the over-bar) including respectively the samples of the unknown function and the forcing function at the quadrature points. The samples of the unknown function are then found as $\overline{\mathrm{j}}=[Z]^{-1} \overline{\mathrm{f}}$. It should be noted that by multiplying both sides of (3.3) by $\omega_{q_{m}}$ as:

$$
\omega_{q_{m}} f\left(\mathbf{r}_{q_{m}}\right)=\sum_{n=1}^{N_{s}} \sum_{q=1}^{N_{q}} \omega_{q_{m}} \omega_{q_{n}} K\left(\mathbf{r}_{q_{m}}, \mathbf{r}_{q_{n}}\right) J\left(\mathbf{r}_{q_{n}}\right) \sqrt{g_{q_{n}}}
$$

the Nyström matrix can be made symmetric.

\subsection{Local Corrections for Singular Kernels}

Most of the kernels occurring in electromagnetics become singular as the test and source subdomains come close to each other. The conventional Nyström method described in the previous section is not useful for singular kernels. As a consequence, the local corrections were devised as special quadrature rules in the regions were the source and test quadrature points are close to each other.

To illustrate the local corrections we assume the test point $\mathbf{r}_{q_{m}}$ is close to the source subdomain $\Omega_{n}$. We define a set of appropriate basis functions $\left\{F_{n}^{k}(\mathbf{r}), k=1, \ldots, N_{f}\right\}$ confined to $\Omega_{n}$. By the aid of the basis functions, a special quadrature rule is defined over $\Omega_{n}:$ 


$$
\sum_{k=1}^{K_{n}} \tilde{\omega}_{q_{m}, q_{n}} F_{n}^{k}\left(\mathbf{r}_{q_{n}}\right)=\int_{\Omega_{n}} K\left(\mathbf{r}_{q_{m}}, \mathbf{r}^{\prime}\right) F_{n}^{k}\left(\mathbf{r}^{\prime}\right) d \Omega^{\prime}
$$

where, $\tilde{\omega}_{q_{m}, q_{n}}$ are the corrected quadrature weights that have to be computed. Gathering (3.6) on $N_{f}$ basis functions:

$$
\left[L_{n}\right] \bar{\omega}_{q_{m}, n}=\bar{k}_{q_{m}, n}
$$

Here, $L_{n}$ is the local correction matrix:

$$
L_{n}=\left[\begin{array}{cccc}
F_{n}^{1}\left(\mathbf{r}_{1_{n}}\right) & F_{n}^{1}\left(\mathbf{r}_{2_{n}}\right) & \ldots & F_{n}^{1}\left(\mathbf{r}_{N_{q, n}}\right) \\
F_{n}^{2}\left(\mathbf{r}_{1_{n}}\right) & F_{n}^{2}\left(\mathbf{r}_{2_{n}}\right) & \ldots & F_{n}^{2}\left(\mathbf{r}_{N_{q, n}}\right) \\
\vdots & \vdots & \ddots & \vdots \\
F_{n}^{N_{f}}\left(\mathbf{r}_{1_{n}}\right) & F_{n}^{N}\left(\mathbf{r}_{2_{n}}\right) & \ldots & F_{n}^{N}\left(\mathbf{r}_{N_{q, n}}\right)
\end{array}\right],
$$

$\bar{\omega}_{q_{m}, n}$ includes the unknown local correction coefficients $\tilde{\omega}_{q_{m}, q_{n}}$, and the $k$ th entry of $\bar{k}_{q_{m}, n}$ is :

$$
\left(\bar{k}_{q_{m}, n}\right)_{k}=\int_{\Omega_{n}} K\left(\mathbf{r}_{q_{m}}, \mathbf{r}^{\prime}\right) F_{n}^{k}\left(\mathbf{r}^{\prime}\right) d \Omega^{\prime}
$$

$L_{n}$ is of size $N_{f} \times N_{q}$. The number of basis functions is commonly chosen the same as the number of quadrature points, $N_{f}=N_{q}$. Therefore, the locally corrected quadrature weights are found as:

$$
\bar{\omega}_{q_{m}, n}=\left[L_{n}\right]^{-1} \bar{k}_{q_{m}, n}
$$

In case $L_{n}$ is not a square matrix, the pseudo-inverse of $L_{n}$ is found by applying SVD or QR decomposition. 
Now, we define the local impedance matrix $\left[z_{m, n}^{\mathrm{LCN}}\right]$ associated with the interaction of the $m$ th test subdomain $\Omega_{m}$ and $n$th source subdomain $\Omega_{n}$. Considering (3.3) when $\Omega_{m}$ and $\Omega_{n}$ are far from each other, the $\left(q_{m}, q_{n}\right)$ th entry of $\left[z_{m, n}^{\mathrm{LCN}}\right]$ is:

$$
\left[z_{m, n}^{\mathrm{LCN}, f a r}\right]_{q_{m}, q_{n}}=\omega_{q_{n}} K\left(\mathbf{r}_{q_{m}}, \mathbf{r}_{q_{n}}\right) \sqrt{g_{q_{n}}}
$$

For near subdomains $\Omega_{m}$ and $\Omega_{n}$, the $\left(q_{m}, q_{n}\right)$ th entry of $\left[z_{m, n}^{\mathrm{LCN}}\right]$ is:

$$
\left[z_{m, n}^{\mathrm{LCN}, \text { near }}\right]_{q_{m}, q_{n}}=\tilde{\omega}_{q_{m}, q_{n}}
$$

where, $\tilde{\omega}_{q_{m}, q_{n}}$ are the weights of the specialized quadrature rule defined in (3.6). As it is observed the far interactions in the LCN system matrix are computed via a single point evaluation of the kernel and the near interactions are calculated by simple algebraic operations demonstrated in (3.10).

\subsection{LCN Applied to Electromagnetic Integral Equations}

In this section, we discuss the locally corrected Nyström discretization of the electromagnetic integral equations described in chapter 2. We start by considering the more general case of (3.1) [45]:

$$
\mathbf{f}^{i n c}(\mathbf{r})=\int_{\Omega} \overline{\mathbf{D}}\left(\mathbf{r}, \mathbf{r}^{\prime}\right) \mathbf{J}\left(\mathbf{r}^{\prime}\right) d \Omega^{\prime}
$$

where $\overline{\mathbf{D}}\left(\mathbf{r}, \mathbf{r}^{\prime}\right)$ is the dyadic kernel related to the integral operator and $\mathbf{J}$ is the unknown current density. $\mathbf{f}^{\text {inc }}(\mathbf{r})$ is the forcing vector function. Choosing the appropriate test vectors $\mathbf{t}(\mathbf{r})$, (3.13) is represented as:

$$
\mathbf{t}(\mathbf{r}) \cdot \mathbf{f}^{i n c}(\mathbf{r})=\mathbf{t}(\mathbf{r}) \cdot \int_{\Omega} \overline{\mathbf{D}}\left(\mathbf{r}, \mathbf{r}^{\prime}\right) \mathbf{J}\left(\mathbf{r}^{\prime}\right) d \Omega^{\prime}
$$


The high order implementation of the LCN method depends on the appropriate meshing of the underlying geometry, the choice of the basis functions, and suitable specification of the test vectors. Consequently, at first we consider the HO geometric discretization of the domain $\Omega$ of the integral operator. Then, we discuss the LCN discretization of the integral operator with dyadic kernel described in (3.14).

\subsubsection{The geometric modeling}

To achieve the HO convergence of the LCN method, the underlying geometry $\Omega$ is modeled by HO curvilinear cells. Consequently, the surfaces in the mesh are discretized by $\mathrm{HO}$ curvilinear triangles or quadrilaterals, and the volumes are discretized by $\mathrm{HO}$ curvilinear hexahedra, prism, or tetrahedrons.

A curvilinear surface cell is uniquely depicted by curvilinear coordinates $\left(u^{1}, u^{2}\right)$. The unitary vectors on this curvilinear face are defined as [11]:

$$
\mathbf{a}_{i}=\frac{\partial \mathbf{r}}{\partial u^{i}}
$$

where, $i=1,2$ and $\mathbf{r}\left(u^{1}, u^{2}\right)$ is the position vector of a point on the curvilinear cell. Consequently, the unitary vectors are tangential to the surface of the curvilinear cell. The differential change in the position vector can thus be expressed as:

$$
d \mathbf{r}=\mathbf{a}_{1} d u^{1}+\mathbf{a}_{2} d u^{2}
$$

The coefficients of the metric tensor are then defined as:

$$
g_{i, j}=\mathbf{a}_{i} \cdot \mathbf{a}_{j}
$$

The unit vector normal to the surface is computed as: 


$$
\hat{\mathbf{n}}=\frac{\mathbf{a}_{1} \times \mathbf{a}_{2}}{\left|\mathbf{a}_{1} \times \mathbf{a}_{2}\right|}
$$

Accordingly, the integral of a vector $\mathbf{F}$ over the surface of the curvilinear cell is represented as:

$$
\begin{aligned}
\int_{S_{c}} \mathbf{F} \cdot d \mathbf{s} & =\int_{S_{c}} \mathbf{F} \cdot\left(\mathbf{a}_{1} \times \mathbf{a}_{2}\right) d u^{1} d u^{2} \\
& =\int_{S_{c}} \mathbf{F} \cdot \hat{\mathbf{n}} \sqrt{g_{s}} d u^{1} d u^{2} \quad \sqrt{ }
\end{aligned}
$$

where,

$$
\sqrt{g_{s}}=\hat{\mathbf{n}} \cdot\left(\mathbf{a}_{1} \times \mathbf{a}_{2}\right)
$$

is the surface Jacobian. The divergence of a vector function $\mathbf{F}\left(u^{1}, u^{2}\right)$ is represented as:

$$
\nabla \cdot \mathbf{F}=\frac{1}{\sqrt{g_{s}}}\left[\frac{\partial}{\partial u^{1}}\left(\mathbf{F} \cdot \mathbf{a}^{1} \sqrt{g_{s}}\right)+\frac{\partial}{\partial u^{2}}\left(\mathbf{F} \cdot \mathbf{a}^{2} \sqrt{g_{s}}\right)\right]
$$

where the complementary set of vectors $\mathbf{a}^{i}, i=1,2$, called reciprocal unitary vectors are defined as:

$$
\mathbf{a}^{1}=\frac{\mathbf{a}_{2} \times \hat{\mathbf{n}}}{\sqrt{g_{s}}} \text { and } \mathbf{a}^{2}=\frac{\hat{\mathbf{n}} \times \mathbf{a}_{1}}{\sqrt{g_{s}}}
$$

The Gradient of a scalar function $f\left(u^{1}, u^{2}\right)$ is then defined as:

$$
\nabla f=\mathbf{a}^{1} \frac{\partial f}{\partial u^{1}}+\mathbf{a}^{2} \frac{\partial f}{\partial u^{2}}
$$

The following relationship holds between the unitary vectors and reciprocal unitary vectors:

$$
\mathbf{a}^{i} \cdot \mathbf{a}_{j}=\delta_{i, j}
$$


where, $\delta_{i, j}$ is the Kronecker delta function. Consequently, the expansion of a vector function $\mathbf{F}\left(u^{1}, u^{2}\right)$ can be derived based on unitary bases as:

$$
\mathbf{F}=f^{1} \mathbf{a}_{1}+f^{2} \mathbf{a}_{2}
$$

where,

$$
f^{i}=\mathbf{F} \cdot \mathbf{a}^{i}
$$

$f^{i}$ are called the contra-variant projection of $\mathbf{F}$. The expansion of $\mathbf{F}$ versus the reciprocal unitary vectors can also be represented as:

$$
\mathbf{F}=f_{1} \mathbf{a}^{1}+f_{2} \mathbf{a}^{2},
$$

where,

$$
f_{i}=\mathbf{F} \cdot \mathbf{a}_{i} .
$$

$f_{i}$ in (3.28) are called co-variant projections of $\mathbf{F}$.

Similarly, a HO curvilinear volume cell is described by the local curvilinear coordinates $\left(u^{1}, u^{2}, u^{3}\right)$. The unitary vectors are defined as:

$$
\mathbf{a}_{i}=\frac{\partial \mathbf{r}}{\partial u^{i}}
$$

where $i=1,2,3$. The coefficients of the metric tensor are defined the same as (3.17). The differential volume $d v$ then can be defined as:

$$
d v=\sqrt{g} d u_{1} d u_{2} d u_{3}
$$

where,

$$
\sqrt{g}=\mathbf{a}_{1} \cdot \mathbf{a}_{2} \times \mathbf{a}_{3},
$$


is the volume Jacobian. Therefore, the integral of a vector function $\mathbf{F}$ over the volume cell $V_{c}$ in curvilinear coordinates can be expressed as:

$$
\int_{V_{c}} \mathbf{F} d v=\int_{V_{c}} \mathbf{F} \sqrt{g} d u^{1} d u^{2} d u^{3}
$$

The divergence of a vector function $\mathbf{F}\left(u^{1}, u^{2}, u^{3}\right)$ is expressed as:

$$
\nabla \cdot \mathbf{F}=\frac{1}{\sqrt{g}}\left[\frac{\partial}{\partial u^{1}}\left(\mathbf{F} \cdot \mathbf{a}^{1} \sqrt{g}\right)+\frac{\partial}{\partial u^{2}}\left(\mathbf{F} \cdot \mathbf{a}^{2} \sqrt{g}\right)+\frac{\partial}{\partial u^{3}}\left(\mathbf{F} \cdot \mathbf{a}^{3} \sqrt{g}\right)\right]
$$

where the reciprocal unitary vectors $\mathbf{a}^{i}, i=1,2,3$ are defined as:

$$
\mathbf{a}^{1}=\frac{\mathbf{a}_{2} \times \mathbf{a}_{3}}{\sqrt{g}}, \quad \mathbf{a}^{2}=\frac{\mathbf{a}_{3} \times \mathbf{a}_{1}}{\sqrt{g}}, \quad \mathbf{a}^{3}=\frac{\mathbf{a}_{1} \times \mathbf{a}_{2}}{\sqrt{g}}
$$

The unitary and reciprocal unitary vectors satisfy the same relationship as (3.24). The Gradient of a scalar function $f\left(u^{1}, u^{2}, u^{3}\right)$ is then defined as:

$$
\nabla f=\mathbf{a}^{1} \frac{\partial f}{\partial u^{1}}+\mathbf{a}^{2} \frac{\partial f}{\partial u^{2}}+\mathbf{a}^{3} \frac{\partial f}{\partial u^{3}}
$$

The co-variant and contra-variant expansions of $\mathbf{F}\left(u^{1}, u^{2}, u^{3}\right)$ are defined respectively as:

$$
\mathbf{F}=f_{1} \mathbf{a}^{1}+f_{2} \mathbf{a}^{2}+f_{3} \mathbf{a}^{3}
$$

and,

$$
\mathbf{F}=f^{1} \mathbf{a}_{1}+f^{2} \mathbf{a}_{2}+f^{3} \mathbf{a}_{3}
$$

where, $f_{i}$ and $f^{i}$ are defined respectively in the same way as (3.26) and (3.28).

\subsubsection{Discretization of the integral operator}

We assume the domain $\Omega$ in (3.14) is described by a mesh with $N_{c}$ cells $\Omega_{n}, n=1, \ldots, N_{c}$. Consequently, (3.14) is rewritten as [45]: 


$$
\mathbf{t}(\mathbf{r}) \cdot \mathbf{f}^{i n c}(\mathbf{r})=\mathbf{t}(\mathbf{r}) \cdot \sum_{n=1}^{N_{c}} \int_{\Omega_{n}} \overline{\mathbf{D}}\left(\mathbf{r}, \mathbf{r}^{\prime}\right) \mathbf{J}\left(\mathbf{r}^{\prime}\right) d \Omega^{\prime}
$$

The current $\mathbf{J}$ on $\Omega_{n}$ is expressed as:

$$
\mathbf{J}\left(\mathbf{r}^{\prime}\right)=\sum_{j=1}^{N_{j}} \mathbf{J}^{j}\left(\mathbf{r}^{\prime}\right) \mathbf{a}_{j}\left(\mathbf{r}^{\prime}\right)
$$

where $\mathbf{a}_{j}\left(\mathbf{r}^{\prime}\right)$ are the unitary vectors. $N_{j}$ is the dimension of the cell $\Omega_{n}$. If $\Omega_{n}$ indicates a surface cell, $N_{j}=2$. If $\Omega_{n}$ is a volume cell, $N_{j}=3 \cdot \mathrm{J}^{j}\left(\mathbf{r}^{\prime}\right)$ are the magnitude of the current samples in unitary directions. Similar to (3.3) by utilizing the appropriate quadrature rule, for far interactions where $\overline{\mathbf{D}}\left(\mathbf{r}, \mathbf{r}^{\prime}\right)$ is not singular (3.38) can be expressed as:

$$
\omega_{q_{m}}^{i} \mathbf{t}_{q_{m}}^{i} \cdot \mathbf{f}^{i n c}\left(\mathbf{r}_{q_{m}}^{i}\right)=\sum_{n=1}^{N_{c}} \sum_{j=1}^{N_{j}} \sum_{q=1}^{N_{q}} \mathbf{J}^{j}\left(\mathbf{r}_{q_{n}}^{j}\right) \sqrt{g_{q_{n}}^{j}} \omega_{q_{m}}^{i} \omega_{q_{n}}^{j} \mathbf{t}_{q_{m}}^{i} \cdot \overline{\mathbf{D}}\left(\mathbf{r}_{q_{m}}^{i}, \mathbf{r}_{q_{n}}^{j}\right) \cdot \mathbf{a}_{j}\left(\mathbf{r}_{q_{n}}^{j}\right)
$$

In (3.40) $i=1, \ldots, N_{i}$ where $N_{i}$ is the dimension of the cell $\Omega_{m} \cdot \mathbf{r}_{q_{n}}^{j}$ are the abscissa of the quadrature rule corresponding to direction $\mathbf{a}_{j}$ on the $n$th cell, and $\mathbf{t}_{q_{m}}^{i}$ are the appropriate test vectors at test point $\mathbf{r}_{q_{m}}^{i}$. As will be discussed later in this chapter, for mixed-order quadrature rules different set of quadrature points are assigned to each unitary direction. $\sqrt{g_{q_{n}}^{j}}$ are the Jacobian at the source quadrature points, and $\omega_{q_{n}}^{j}$ is the quadrature weight corresponding to $\mathbf{r}_{q_{n}}^{j}$. It should be noted that the multiplication of the test quadrature weights $\omega_{q_{m}}^{i}$ to the both sides of (3.40) are necessary to complete the connection between the LCN and MOM that will be discussed in chapter 5. Defining:

$$
\mathrm{j}^{j}\left(\mathbf{r}_{q_{n}}^{j}\right)=\sqrt{g_{q_{n}}^{j}} \mathrm{~J}^{j}\left(\mathbf{r}_{q_{n}}^{j}\right)
$$


Equation (3.40) is rewritten as:

$$
\omega_{q_{m}}^{i} \mathbf{t}_{q_{m}}^{i} \cdot \mathbf{f}^{i n c}\left(\mathbf{r}_{q_{m}}^{i}\right)=\sum_{n=1}^{N_{c}} \sum_{j=1}^{N_{j}} \sum_{q=1}^{N_{q}} \mathrm{j}^{j}\left(\mathbf{r}_{q_{n}}^{j}\right) \omega_{q_{m}}^{i} \omega_{q_{n}}^{j} \mathbf{t}_{q_{m}}^{i} \cdot \overline{\mathbf{D}}\left(\mathbf{r}_{q_{m}}^{i}, \mathbf{r}_{q_{n}}^{j}\right) \cdot \mathbf{a}_{j}\left(\mathbf{r}_{q_{n}}^{j}\right)
$$

It should be noted that the scaling by Jacobian makes the unknown vector divergence-less. If $\overline{\mathbf{D}}\left(\mathbf{r}, \mathbf{r}^{\prime}\right)$ is a regular kernel, collecting (3.42) at all test quadrature points results in a square linear system of equations. However, typically when $\mathbf{r}_{q_{m}}^{i}$ approaches $\mathbf{r}_{q_{n}}^{j}$, $\overline{\mathbf{D}}\left(\mathbf{r}_{q_{m}}^{i}, \mathbf{r}_{q_{n}}^{j}\right)$ becomes singular. As a consequence, a set of suitable basis functions:

$$
\mathbf{F}_{n}^{k, j}(\mathbf{r})=F_{n}^{k, j}(\mathbf{r}) \mathbf{a}_{j}(\mathbf{r})
$$

are defined over the cell $\Omega_{n}$ where, $k=1, \ldots, N_{q}$, and $j=1, \ldots, N_{j}$. In a way similar to (3.6), a special quadrature rule is introduced:

$$
\sum_{k=1}^{K_{n}} \tilde{\omega}_{q_{m}, q_{n}}^{i, j} F_{n}^{k, j}\left(\mathbf{r}_{q_{n}}^{j}\right)=\omega_{q_{m}}^{i} \mathbf{t}\left(\mathbf{r}_{q_{m}}^{i}\right) \cdot \int_{\Omega_{n}} \overline{\mathbf{D}}\left(\mathbf{r}_{q_{m}}^{i}, \mathbf{r}^{\prime}\right) \cdot \mathbf{F}_{n}^{k, j}\left(\mathbf{r}^{\prime}\right) d \Omega^{\prime}
$$

where, $\tilde{\omega}_{q_{m}, q_{n}}^{i, j}$ are local correction coefficients. Gathering (3.44) for all the test quadrature points on $\Omega_{m}$ and all the current basis functions on $\Omega_{n}$ results in a matrix equation to solve for the unknown coefficients $\tilde{\omega}_{q_{m}, q_{n}}^{i, j}$. Therefore the matrix form of (3.44) can be expressed as:

$$
\left[L_{n}\right] \bar{\omega}_{q_{m}, n}^{i}=\bar{d}_{q_{m}, n}^{i}
$$

Assuming that the source cell $\Omega_{n}$ is a surface cell, $L_{n}$ is a block diagonal matrix expressed as:

$$
\left[L_{n}\right]=\left[\begin{array}{cc}
{\left[L_{n}^{1}\right]} & {[0]} \\
{[0]} & {\left[L_{n}^{2}\right]}
\end{array}\right]
$$

where $\left[L_{n}^{j}\right]$ is a $N_{q} \times N_{q}$ matrix with $(k, q)$ th entry: 


$$
\left[L_{n}^{j}\right]_{k, q}=F_{n}^{k, j}\left(\mathbf{r}_{q_{n}}^{j}\right)
$$

$\bar{\omega}_{q_{m}, n}^{i}$ and $\bar{d}_{q_{m}, n}^{i}$ in (3.45) are then vectors of length $2 N_{q}$ defined as:

$$
\bar{\omega}_{q_{m}, n}^{i}=\left[\begin{array}{c}
\bar{\omega}_{q_{m}, n}^{i, 1} \\
\bar{\omega}_{q_{m}, n}^{i, n}
\end{array}\right] \text {, and } \quad \bar{d}_{q_{m}, n}^{i}=\left[\begin{array}{c}
\bar{d}_{q_{m}, n}^{i, 1} \\
\bar{d}_{q_{m}, n}^{i, 2}
\end{array}\right]
$$

$\bar{\omega}_{q_{m}, n}^{i, j}, j=1,2$, comprises the unknown local correction coefficients. The $q$ th entry of $\bar{\omega}_{q_{m}, n}^{i, j}$ is:

$$
\left(\bar{\omega}_{q_{m}, n}^{i, j}\right)_{q}=\bar{\omega}_{q_{m}, q_{n}}^{i, j}
$$

and the $k$ th entry of $\bar{d}_{q_{m}, n}^{i, j}$ is:

$$
\left(\bar{d}_{q_{m}, n}^{i, j}\right)_{k}=\omega_{q_{m}}^{i} \mathbf{t}_{q_{m}}^{i} \cdot \int_{\Omega_{n}} \overline{\mathbf{D}}\left(\mathbf{r}_{q_{m}}^{i}, \mathbf{r}^{\prime}\right) \cdot \mathbf{F}_{n}^{k, j}\left(\mathbf{r}^{\prime}\right) d \Omega^{\prime}
$$

In case of a volume cell $\Omega_{n}, L_{n}$ is of the form:

$$
\left[L_{n}\right]=\left[\begin{array}{ccc}
{\left[L_{n}^{1}\right]} & {[0]} & {[0]} \\
{[0]} & {\left[L_{n}^{2}\right]} & {[0]} \\
{[0]} & {[0]} & {\left[L_{n}^{3}\right.}
\end{array}\right],
$$

where, $\left[L_{n}^{j}\right]$ was defined in (3.47). Similar to (3.48), $\bar{\omega}_{q_{m}, n}^{i}$ and $\bar{d}_{q_{m}, n}^{i}$ for volume cells are vectors of length $3 N_{q}$ represented as:

$$
\bar{\omega}_{q_{m}, n}^{i}=\left[\begin{array}{c}
\bar{\omega}_{q_{m}, n}^{i, 1} \\
\bar{\omega}_{q_{m}, n}^{i, 2} \\
\bar{\omega}_{q_{m}, n}^{i, 3}
\end{array}\right] \text {, and } \quad \bar{d}_{q_{m}, n}^{i}=\left[\begin{array}{c}
\bar{d}_{q_{m}, n}^{i, 1} \\
\bar{d}_{q_{m}, n}^{i, n} \\
\bar{d}_{q_{m}, n}^{i, 3}
\end{array}\right]
$$

where, the elements of $\bar{\omega}_{q_{m}, n}^{i, j}$ and $\bar{d}_{q_{m}, n}^{i, j}$ are defined in the same way as (3.49) and (3.50). Consequently, the local correction coefficients are obtained by: 


$$
\bar{\omega}_{q_{m}, n}^{i}=\left[L_{n}\right]^{-1} \bar{d}_{q_{m}, n}^{i}
$$

The discussion for the volume integral equation is very similar. Based on (3.42) and (3.53) the full LCN discretization of (3.14) can thus be expressed as:

$$
\left[Z^{\mathrm{LCN}}\right] \overline{\mathrm{j}}=\overline{\mathrm{f}}
$$

Assuming that all cells have the same dimension (i.e. they are all surface or volume cells), $\left[\mathrm{Z}^{\mathrm{LCN}}\right]$ is of size $N \times N$ where, $N=N_{i} N_{q} N_{c}$. Without loss of generality, a single excitation is assumed so that $\bar{j}$ and $\bar{f}$ are column vectors (indicated by the over-bar) which can be represented as concatenation of $N_{c}$ vectors:

$$
\overline{\mathrm{j}}=\left[\begin{array}{c}
\bar{J}_{1} \\
\vdots \\
\bar{J}_{n} \\
\vdots \\
\bar{J}_{N_{c}}
\end{array}\right], \quad \overline{\mathrm{f}}=\left[\begin{array}{c}
\bar{f}_{1} \\
\vdots \\
\overline{f_{n}} \\
\vdots \\
\bar{f}_{N_{c}}
\end{array}\right] \text {, }
$$

where, the vectors $\bar{J}_{n}$ and $\bar{f}_{n}$ are the current and field samples, respectively, on the $n$th cell. Supposing that $\Omega_{n}$ are surface cells (while the extension to volume cells is straightforward), $\bar{J}_{n}$ and $\bar{f}_{n}$ can be expressed as:

$$
\bar{J}_{n}=\left[\begin{array}{c}
\bar{J}_{n}^{1} \\
\bar{J}_{n}^{2}
\end{array}\right] \text {, and } \bar{f}_{n}=\left[\begin{array}{c}
\bar{f}_{n}^{1} \\
\bar{f}_{n}^{2}
\end{array}\right] \text {. }
$$

where, $\bar{J}_{n}^{i}$ and $\bar{f}_{n}^{i}, i=1,2$, are column vectors with length $N_{q}$, and the $q$ th entry of $\bar{J}_{n}^{i}$ is $\left(\bar{J}_{n}^{i}\right)_{q}=\mathrm{J}^{i}\left(\mathbf{r}_{q_{n}}^{i}\right)$. The $q$ th entry of $\bar{f}_{n}^{i}$ is represented as:

$$
\left(\bar{f}_{n}^{i}\right)_{q}=\omega_{q_{n}}^{i} \mathbf{t}_{q_{n}}^{j} \cdot \mathbf{f}^{i n c}\left(\mathbf{r}_{q_{n}}^{i}\right)
$$


For a surface integral equation represented by (3.14), the corresponding $Z^{\text {LCN }}$ in (3.54) is the usual LCN impedance matrix of size $2 N_{q} N_{c} \times 2 N_{q} N_{c}$ that comprises all local LCN impedance matrices, $\left[z_{m, n}^{\mathrm{LCN}}\right]$. The $\left[z_{m, n}^{\mathrm{LCN}}\right]$ describe the interaction between the observation cell $m$ and the source cell $n$ and are represented as:

$$
\left[z_{m, n}^{\mathrm{LCN}}\right]=\left[\begin{array}{ll}
{\left[z_{m, n}^{1,1}\right]} & {\left[z_{m, n}^{1,2}\right]} \\
{\left[z_{m, n}^{2,1}\right]} & {\left[z_{m, n}^{2,2}\right]}
\end{array}\right],
$$

where, the $z_{m, n}^{i, j}$ blocks in (3.58) are $N_{q} \times N_{q}$ matrices obtained by evaluating the kernel integrals based on (3.42) or (3.44). For sufficiently separated source and observation cells, considering (3.42) the entry $\left(q_{m}, q_{n}\right)$ of $z_{m, n}^{i, j}$ is simply:

$$
\left[z_{m, n}^{i, j}\right]_{q_{m}, q_{n}}=\omega_{q_{m}}^{i} \omega_{q_{n}}^{j} \mathbf{t}_{q_{m}}^{i} \cdot \overline{\mathbf{D}}\left(\mathbf{r}_{q_{m}}^{i}, \mathbf{r}_{q_{n}}^{j}\right) \cdot \mathbf{a}_{j}\left(\mathbf{r}_{q_{n}}^{j}\right)
$$

For source and test cells that are near to each other, $\overline{\mathbf{D}}\left(\mathbf{r}, \mathbf{r}^{\prime}\right)$ varies rapidly, and the integration in (3.38) cannot be accurately represented by a fixed point quadrature rule. Applying the local corrections, the entry $\left(q_{m}, q_{n}\right)$ of $z_{m, n}^{i, j}$ is expressed as:

$$
\left[z_{m, n}^{i, j}\right]_{q_{m}, q_{n}}=\tilde{\omega}_{q_{m}, q_{n}}^{i, j}
$$

where, $\tilde{\omega}_{q_{m}, q_{n}}^{i, j}$ was defined in (3.44).

As discussed in the previous chapter, the integral operators for SIE and VIE can be expressed in terms of the $\mathbf{L}$ and $\mathbf{K}$ operators. Therefore, the LCN discretization of the $\mathbf{L}$ and $\mathbf{K}$ operators provide the necessary tools to discretize surface and volume integral equations described in chapter 2.

\section{A. LCN discretization of the $\mathbf{L}$ operator}

We consider the $\mathbf{L}$ operator represented in (2.65) operating on $\mathbf{J}$ : 


$$
\mathbf{L}(\mathbf{J})=\int_{\Omega} \overline{\mathbf{I}}\left(\mathbf{r}, \mathbf{r}^{\prime}\right) \cdot \mathbf{J}\left(\mathbf{r}^{\prime}\right) d \Omega^{\prime},
$$

where, $\mathbf{J}$ was defined in (3.39) and $\overline{\mathbf{I}}\left(\mathbf{r}, \mathbf{r}^{\prime}\right)$ is the $\mathbf{L}$ operator dyadic kernel defined as:

$$
\overline{\mathbf{I}}\left(\mathbf{r}, \mathbf{r}^{\prime}\right)=-j k_{1}\left[\overline{\mathbf{I}}+\frac{1}{k_{1}^{2}} \nabla \nabla\right] G\left(\mathbf{r}, \mathbf{r}^{\prime}\right)
$$

Based on (3.38), in order to obtain the LCN implementation of the $\mathbf{L}$ operator we need to evaluate:

$$
\mathbf{t}_{q_{m}}^{i} \cdot \mathbf{L}(\mathbf{J})=\mathbf{t}_{q_{m}}^{i} \cdot \sum_{n=1}^{N_{c}} \int_{\Omega_{n}} \overline{\mathbf{I}}\left(\mathbf{r}_{q_{m}}^{i}, \mathbf{r}^{\prime}\right) \cdot \mathbf{J}\left(\mathbf{r}^{\prime}\right) d \Omega^{\prime},
$$

where, $\mathbf{r}_{q_{m}}^{i}$ are the test points on $\Omega_{m}$. For test and source cells, $\Omega_{m}$ and on $\Omega_{n}$, that are sufficiently far from each other (3.63) can be computed based on (3.42) by single point evaluations of $\overline{\mathbf{I}}\left(\mathbf{r}, \mathbf{r}^{\prime}\right)$ at source and test quadrature points:

$$
\mathbf{t}_{q_{m}}^{i} \cdot \mathbf{L}(\mathbf{J})=\sum_{n=1}^{N_{c}} \sum_{j=1}^{N_{j}} \sum_{q=1}^{N_{q}} \mathrm{j}^{j}\left(\mathbf{r}_{q_{n}}^{j}\right) \omega_{q_{m}}^{i} \omega_{q_{n}}^{j} \mathbf{t}_{q_{m}}^{i} \cdot \overline{\mathbf{l}}\left(\mathbf{r}_{q_{m}}^{i}, \mathbf{r}_{q_{n}}^{j}\right) \cdot \mathbf{a}_{j}\left(\mathbf{r}_{q_{n}}^{j}\right)
$$

where, $\mathrm{j}^{j}\left(\mathbf{r}_{q_{n}}^{j}\right)$ are the unknown current samples scaled by the Jacobian at the source quadrature points. Substituting (2.41) in (3.62), the following useful formulation holds that is applied to calculate (3.64):

$$
\begin{aligned}
\mathbf{t}_{q_{m}}^{i} \cdot \overline{\mathbf{I}}\left(\mathbf{r}_{q_{m}}^{i}, \mathbf{r}_{q_{n}}^{j}\right) \cdot \mathbf{a}_{j}\left(\mathbf{r}_{q_{n}}^{j}\right)=- & j k_{1} \frac{\exp \left(-j k_{1} R\right)}{4 \pi R} . \\
& \left\{\left(\mathbf{t}_{q_{m}}^{i} \cdot \mathbf{a}_{j}\left(\mathbf{r}_{q_{n}}^{j}\right)\right)\left(1-\frac{1}{k_{1}^{2}}\left(\frac{j k_{1} R+1}{R^{2}}\right)\right)-\right. \\
& \left.\frac{\left(\mathbf{t}_{q_{m}}^{i} \cdot \mathbf{R}\right)\left(\mathbf{R} \cdot \mathbf{a}_{j}\left(\mathbf{r}_{q_{n}}^{j}\right)\right)}{R^{2}}\left(1-\frac{3}{k_{1}^{2}}\left(\frac{j k_{1} R+1}{R^{2}}\right)\right)\right\},
\end{aligned}
$$

where, 


$$
\mathbf{R}=\mathbf{r}_{q_{m}}^{i}-\mathbf{r}_{q_{n}}^{j} \text {, and } R=\left|\mathbf{r}_{q_{m}}^{i}-\mathbf{r}_{q_{n}}^{j}\right|
$$

For near test and source cells, $\Omega_{m}$ and $\Omega_{n}, \overline{\mathbf{I}}\left(\mathbf{r}, \mathbf{r}^{\prime}\right)$ becomes singular and (3.64) is not useful anymore. Consequently, in order to find the local correction coefficients by the application of (3.44), we need to calculate the following integration:

$$
\begin{aligned}
& \mathbf{t}_{q_{m}}^{i} \cdot \int_{\Omega_{n}} \overline{\mathbf{I}}\left(\mathbf{r}_{q_{m}}^{i}, \mathbf{r}^{\prime}\right) \cdot \mathbf{F}_{n}^{k, j}\left(\mathbf{r}^{\prime}\right) d \Omega^{\prime}= \\
& \mathbf{t}_{q_{m}}^{i} \cdot \int_{\Omega_{n}}-\left.j k_{1}\left(\left[\overline{\mathbf{I}} \cdot+\frac{1}{k_{1}^{2}} \nabla \nabla \cdot\right] G\left(\mathbf{r}, \mathbf{r}^{\prime}\right) \cdot \mathbf{F}_{n}^{k, j}\left(\mathbf{r}^{\prime}\right)\right)\right|_{\mathbf{r}=\mathbf{r}_{q_{m}}^{i}} d \Omega^{\prime}
\end{aligned}
$$

where, $\mathbf{F}_{n}^{k, i}$ are the current basis functions defined in (3.43). The integrand in (3.67) is hyper-singular as $\mathbf{r} \rightarrow \mathbf{r}^{\prime}$; therefore, the hyper-singularity should be removed so that the integration becomes numerically manageable [35]. At the first step (3.67) is rewritten as:

$$
\begin{array}{r}
\mathbf{t}_{q_{m}}^{i} \cdot \int_{\Omega_{n}} \overline{\mathbf{I}}\left(\mathbf{r}_{q_{m}}^{i}, \mathbf{r}^{\prime}\right) \cdot \mathbf{F}_{n}^{k, j}\left(\mathbf{r}^{\prime}\right) d \Omega^{\prime}=-j k_{1} \mathbf{t}_{q_{m}}^{i} \cdot \int_{\Omega_{n}} G\left(\mathbf{r}_{q_{m}}^{i}, \mathbf{r}^{\prime}\right) \cdot \mathbf{F}_{n}^{k, j}\left(\mathbf{r}^{\prime}\right) d \Omega^{\prime}- \\
\left.\frac{j}{k_{1}} \mathbf{t}_{q_{m}}^{i} \cdot\left(\int_{\Omega_{n}} \nabla \nabla \cdot\left(G\left(\mathbf{r}, \mathbf{r}^{\prime}\right) \cdot \mathbf{F}_{n}^{k, j}\left(\mathbf{r}^{\prime}\right)\right) d \Omega^{\prime}\right)\right|_{\mathbf{r}=\mathbf{r}_{q_{m}}^{i}}
\end{array}
$$

By utilizing the identity $\nabla \cdot(b \mathbf{a})=b \nabla \cdot \mathbf{a}+\nabla b \cdot \mathbf{a}$, and noting that $\nabla G\left(\mathbf{r}, \mathbf{r}^{\prime}\right)=-\nabla^{\prime} G\left(\mathbf{r}, \mathbf{r}^{\prime}\right)$, (3.68) is represented as:

$$
\begin{array}{r}
\mathbf{t}_{q_{m}}^{i} \cdot \int_{\Omega_{n}} \overline{\mathbf{I}}\left(\mathbf{r}_{q_{m}}^{i}, \mathbf{r}^{\prime}\right) \cdot \mathbf{F}_{n}^{k, j}\left(\mathbf{r}^{\prime}\right) d \Omega^{\prime}=-j k_{1} \mathbf{t}_{q_{m}}^{i} \cdot \int_{\Omega_{n}} G\left(\mathbf{r}_{q_{m}}^{i}, \mathbf{r}^{\prime}\right) \cdot \mathbf{F}_{n}^{k, j}\left(\mathbf{r}^{\prime}\right) d \Omega^{\prime}+ \\
\left.\frac{j}{k_{1}} \mathbf{t}_{q_{m}}^{i} \cdot\left(\nabla \int_{\Omega_{n}} \nabla^{\prime} G\left(\mathbf{r}, \mathbf{r}^{\prime}\right) \cdot \mathbf{F}_{n}^{k, j}\left(\mathbf{r}^{\prime}\right) d \Omega^{\prime}\right)\right|_{\mathbf{r}=\mathbf{r}_{q_{m}}^{i}}
\end{array}
$$

By applying $\nabla b \cdot \mathbf{a}=b \nabla \cdot \mathbf{a}-\nabla \cdot(b \mathbf{a})$ we can write:

$$
\nabla^{\prime} G\left(\mathbf{r}, \mathbf{r}^{\prime}\right) \cdot \mathbf{F}_{n}^{k, j}\left(\mathbf{r}^{\prime}\right)=\nabla^{\prime} \cdot\left(G\left(\mathbf{r}, \mathbf{r}^{\prime}\right) \mathbf{F}_{n}^{k, j}\left(\mathbf{r}^{\prime}\right)\right)-G\left(\mathbf{r}, \mathbf{r}^{\prime}\right) \nabla^{\prime} \cdot \mathbf{F}_{n}^{k, j}\left(\mathbf{r}^{\prime}\right)
$$

Substituting (3.70) in (3.69): 


$$
\begin{aligned}
\mathbf{t}_{q_{m}}^{i} \cdot \int_{\Omega_{n}} \overline{\mathbf{I}}\left(\mathbf{r}_{q_{m}}^{i}, \mathbf{r}^{\prime}\right) & \cdot \mathbf{F}_{n}^{k, j}\left(\mathbf{r}^{\prime}\right) d \Omega^{\prime}=-j k_{1} \mathbf{t}_{q_{m}}^{i} \cdot \int_{\Omega_{n}} G\left(\mathbf{r}_{q_{m}}^{i}, \mathbf{r}^{\prime}\right) \cdot \mathbf{F}_{n}^{k, j}\left(\mathbf{r}^{\prime}\right) d \Omega^{\prime} \\
& -\left.\frac{j}{k_{1}} \mathbf{t}_{q_{m}}^{i} \cdot \int_{\Omega_{n}} \nabla G\left(\mathbf{r}, \mathbf{r}^{\prime}\right)\right|_{\mathbf{r}=\mathbf{r}_{q_{m}}^{i}} \nabla^{\prime} \cdot \mathbf{F}_{n}^{k, j}\left(\mathbf{r}^{\prime}\right) d \Omega^{\prime} \\
& +\frac{j}{k_{1}} \mathbf{t}_{q_{m}}^{i} \cdot \nabla \int_{\Omega_{n}} \nabla^{\prime} \cdot\left(G\left(\mathbf{r}, \mathbf{r}^{\prime}\right) \mathbf{F}_{n}^{k, j}\left(\mathbf{r}^{\prime}\right)\right) d \Omega^{\prime}
\end{aligned}
$$

Using the divergence theorem, (3.71) is expressed as:

$$
\begin{aligned}
\mathbf{t}_{q_{m}}^{i} \cdot \int_{\Omega_{n}} \overline{\mathbf{I}}\left(\mathbf{r}_{q_{m}}^{i}, \mathbf{r}^{\prime}\right) & \cdot \mathbf{F}_{n}^{k, j}\left(\mathbf{r}^{\prime}\right) d \Omega^{\prime}=-j k_{1} \mathbf{t}_{q_{m}}^{i} \cdot \int_{\Omega_{n}} G\left(\mathbf{r}_{q_{m}}^{i}, \mathbf{r}^{\prime}\right) \cdot \mathbf{F}_{n}^{k, j}\left(\mathbf{r}^{\prime}\right) d \Omega^{\prime} \\
& -\left.\frac{j}{k_{1}} \mathbf{t}_{q_{m}}^{i} \cdot \int_{\Omega_{n}} \nabla G\left(\mathbf{r}, \mathbf{r}^{\prime}\right)\right|_{\mathbf{r}=\mathbf{r}_{q_{m}}^{i}} \nabla^{\prime} \cdot \mathbf{F}_{n}^{k, j}\left(\mathbf{r}^{\prime}\right) d \Omega^{\prime} \\
& +\frac{j}{k_{1}} \oint_{\partial \Omega_{n}}\left(\left.\mathbf{t}_{q_{m}}^{i} \cdot \nabla G\left(\mathbf{r}, \mathbf{r}^{\prime}\right)\right|_{\mathbf{r}=\mathbf{r}_{q_{m}}^{i}}\right)\left(\mathbf{F}_{n}^{k, j}\left(\mathbf{r}^{\prime}\right) \cdot \mathbf{e}_{n}\left(\mathbf{r}^{\prime}\right)\right) d \Gamma^{\prime}
\end{aligned}
$$

where, $\partial \Omega_{n}$ is the boundary enclosing the cell $\Omega_{n}$. For a surface cell $\Omega_{n}, \partial \Omega_{n}$ is a contour and $d \Gamma^{\prime}=d l^{\prime}$. Similarly, for a volume cell $\Omega_{n}, \partial \Omega_{n}$ is a surface and $d \Gamma^{\prime}=d s^{\prime}$. $\mathbf{e}_{n}\left(\mathbf{r}^{\prime}\right)$ is the outward normal to $\partial \Omega_{n}$ at $\mathbf{r}^{\prime}$. The integrand of the second term in the right side of (3.72) still is hyper-singular. In order to remove the hyper-singularity, we introduce an auxiliary function $\mathbf{D}_{m, n}^{i, j}\left(\mathbf{r}^{\prime}\right)$ such that:

$$
\mathbf{D}_{m, n}^{i, j}\left(\mathbf{r}^{\prime}\right)=\left.\frac{\left(\mathbf{t}_{q_{m}}^{i} \cdot \mathbf{a}^{1}\left(\mathbf{r}_{q_{m}}^{i}\right)\right) \mathbf{a}_{1}\left(\mathbf{r}^{\prime}\right)+\left(\mathbf{t}_{q_{m}}^{i} \cdot \mathbf{a}^{2}\left(\mathbf{r}_{q_{m}}^{i}\right)\right) \mathbf{a}_{2}\left(\mathbf{r}^{\prime}\right)}{\sqrt{g^{\prime}}}\left(\sqrt{g^{\prime}} \nabla^{\prime} \cdot \mathbf{F}_{n}^{k, j}\left(\mathbf{r}^{\prime}\right)\right)\right|_{\mathbf{r}^{\prime}=\mathbf{r}_{q_{m}}^{i}}
$$

where, $\sqrt{g^{\prime}}$ is the Jacobian at $\mathbf{r}^{\prime} . \mathbf{a}^{1}$ and $\mathbf{a}^{2}$ are the reciprocal unitary vectors which will be defined later in this chapter. Therefore, at $\mathbf{r}^{\prime}=\mathbf{r}_{q_{m}}^{i}$ :

$$
\mathbf{D}_{m, n}^{i, j}\left(\mathbf{r}_{q_{m}}^{i}\right)=\left.\mathbf{t}_{q_{m}}^{i} \nabla^{\prime} \cdot \mathbf{F}_{n}^{k, j}\left(\mathbf{r}^{\prime}\right)\right|_{\mathbf{r}^{\prime}=\mathbf{r}_{q_{m}}^{i}}
$$

Consequently, we rewrite (3.72) as: 


$$
\begin{aligned}
\mathbf{t}_{q_{m}}^{i} \cdot \int_{\Omega_{n}} \overline{\mathbf{I}}\left(\mathbf{r}_{q_{m}}^{i}, \mathbf{r}^{\prime}\right) & \cdot \mathbf{F}_{n}^{k, j}\left(\mathbf{r}^{\prime}\right) d \Omega^{\prime}=-j k_{1} \mathbf{t}_{q_{m}}^{i} \cdot \int_{\Omega_{n}} G\left(\mathbf{r}_{q_{m}}^{i}, \mathbf{r}^{\prime}\right) \cdot \mathbf{F}_{n}^{k, j}\left(\mathbf{r}^{\prime}\right) d \Omega^{\prime} \\
& -\left.\frac{j}{k_{1}} \int_{\Omega_{n}} \nabla G\left(\mathbf{r}, \mathbf{r}^{\prime}\right)\right|_{\mathbf{r}=\mathbf{r}_{q_{m}}^{i}}\left(\mathbf{t}_{q_{m}}^{i} \nabla^{\prime} \cdot \mathbf{F}_{n}^{k, j}\left(\mathbf{r}^{\prime}\right)-\mathbf{D}_{m, n}^{i, j}\left(\mathbf{r}^{\prime}\right)\right) d \Omega^{\prime} \\
& -\left.\frac{j}{k_{1}} \int_{\Omega_{n}} \nabla G\left(\mathbf{r}, \mathbf{r}^{\prime}\right)\right|_{\mathbf{r}=\mathbf{r}_{q_{m}}^{i}} \mathbf{D}_{m, n}^{i, j}\left(\mathbf{r}^{\prime}\right) d \Omega^{\prime} \\
& +\frac{j}{k_{1}} \oint_{\partial \Omega_{n}}\left(\left.\mathbf{t}_{q_{m}}^{i} \cdot \nabla G\left(\mathbf{r}, \mathbf{r}^{\prime}\right)\right|_{\mathbf{r}=\mathbf{r}_{q_{m}}^{i}}\right)\left(\mathbf{F}_{n}^{k, j}\left(\mathbf{r}^{\prime}\right) \cdot \mathbf{e}_{n}\left(\mathbf{r}^{\prime}\right)\right) d \Gamma^{\prime}
\end{aligned}
$$

As a result, the integrand in the second term of (3.75) is not hyper singular anymore. The third term, however, needs further treatment. By applying $\nabla b \cdot \mathbf{a}=b \nabla \cdot \mathbf{a}-\nabla \cdot(b \mathbf{a})$ and noting that $\nabla G\left(\mathbf{r}, \mathbf{r}^{\prime}\right)=-\nabla^{\prime} G\left(\mathbf{r}, \mathbf{r}^{\prime}\right)$, we have:

$$
\begin{aligned}
\left.\int_{\Omega_{n}} \nabla G\left(\mathbf{r}, \mathbf{r}^{\prime}\right)\right|_{\mathbf{r}=\mathbf{r}_{q_{m}}^{i}} & \mathbf{D}_{m, n}^{i, j}\left(\mathbf{r}^{\prime}\right) d \Omega^{\prime}= \\
& -\int_{\Omega_{n}}\left[\nabla^{\prime} \cdot\left(\mathbf{D}_{m, n}^{i, j}\left(\mathbf{r}^{\prime}\right) G\left(\mathbf{r}_{q_{m}}^{i}, \mathbf{r}^{\prime}\right)\right)-G\left(\mathbf{r}_{q_{m}}^{i}, \mathbf{r}^{\prime}\right) \nabla^{\prime} \cdot \mathbf{D}_{m, n}^{i, j}\left(\mathbf{r}^{\prime}\right)\right] d \Omega^{\prime}
\end{aligned}
$$

From (3.73), it can be easily verified that $\nabla^{\prime} \cdot \mathbf{D}_{m, n}^{i, j}\left(\mathbf{r}^{\prime}\right)=0$. Therefore, utilizing the divergence theorem (3.76) is expressed as:

$$
\left.\int_{\Omega_{n}} \nabla G\left(\mathbf{r}, \mathbf{r}^{\prime}\right)\right|_{\mathbf{r}=\mathbf{r}_{q_{m}}^{i}} \mathbf{D}_{m, n}^{i, j}\left(\mathbf{r}^{\prime}\right) d \Omega^{\prime}=-\oint_{\partial \Omega_{n}} G\left(\mathbf{r}_{q_{m}}^{i}, \mathbf{r}^{\prime}\right)\left(\mathbf{D}_{m, n}^{i, j}\left(\mathbf{r}^{\prime}\right) \cdot \mathbf{e}_{n}\left(\mathbf{r}^{\prime}\right)\right) d \Gamma^{\prime}
$$

Finally, substituting, (3.77) in (3.75) we have:

$$
\begin{aligned}
\mathbf{t}_{q_{m}}^{i} \cdot \int_{\Omega_{n}} \overline{\mathbf{I}}\left(\mathbf{r}_{q_{m}}^{i}, \mathbf{r}^{\prime}\right) & \cdot \mathbf{F}_{n}^{k, j}\left(\mathbf{r}^{\prime}\right) d \Omega^{\prime}=-j k_{1} \mathbf{t}_{q_{m}}^{i} \cdot \int_{\Omega_{n}} G\left(\mathbf{r}_{q_{m}}^{i}, \mathbf{r}^{\prime}\right) \cdot \mathbf{F}_{n}^{k, j}\left(\mathbf{r}^{\prime}\right) d \Omega^{\prime} \\
& -\left.\frac{j}{k_{1}} \int_{\Omega_{n}} \nabla G\left(\mathbf{r}, \mathbf{r}^{\prime}\right)\right|_{\mathbf{r}=\mathbf{r}_{q_{m}}^{i}}\left(\mathbf{t}_{q_{m}}^{i} \nabla^{\prime} \cdot \mathbf{F}_{n}^{k, j}\left(\mathbf{r}^{\prime}\right)-\mathbf{D}_{m, n}^{i, j}\left(\mathbf{r}^{\prime}\right)\right) d \Omega^{\prime} \\
& +\frac{j}{k_{1}} \oint_{\partial \Omega_{n}} G\left(\mathbf{r}_{q_{m}}^{i}, \mathbf{r}^{\prime}\right)\left(\mathbf{D}_{m, n}^{i, j}\left(\mathbf{r}^{\prime}\right) \cdot \mathbf{e}_{n}\left(\mathbf{r}^{\prime}\right)\right) d \Gamma^{\prime} \\
& +\frac{j}{k_{1}} \oint_{\partial \Omega_{n}}\left(\left.\mathbf{t}_{q_{m}}^{i} \cdot \nabla G\left(\mathbf{r}, \mathbf{r}^{\prime}\right)\right|_{\mathbf{r}=\mathbf{r}_{q_{m}}^{i}}\right)\left(\mathbf{F}_{n}^{k, j}\left(\mathbf{r}^{\prime}\right) \cdot \mathbf{e}_{n}\left(\mathbf{r}^{\prime}\right)\right) d \Gamma^{\prime}
\end{aligned}
$$


Now, the boundary integrations are not singular, and the first two terms have a $1 /\left|\mathbf{r}-\mathbf{r}^{\prime}\right|$ singularity which are numerically manageable by using Duffy transform [46].

\section{B. LCN discretization of the $\mathbf{K}$ operator}

The $\mathbf{K}$ operator defined in (2.66) operating on $\mathbf{J}$ is expressed as:

$$
\mathbf{K}(\mathbf{J})=\int_{\Omega} \overline{\mathbf{k}}\left(\mathbf{r}, \mathbf{r}^{\prime}\right) \cdot \mathbf{J}\left(\mathbf{r}^{\prime}\right) d \Omega^{\prime}
$$

where, $\overline{\mathbf{k}}\left(\mathbf{r}, \mathbf{r}^{\prime}\right)$ is the dyadic kernel associated with the $\mathbf{K}$ operator such that:

$$
\int_{\Omega} \overline{\mathbf{k}}\left(\mathbf{r}, \mathbf{r}^{\prime}\right) \cdot \mathbf{J}\left(\mathbf{r}^{\prime}\right) d \Omega^{\prime}=\int_{\Omega} \nabla G\left(\mathbf{r}, \mathbf{r}^{\prime}\right) \times \mathbf{J}\left(\mathbf{r}^{\prime}\right) d \Omega^{\prime}
$$

where, $\mathbf{J}$ was defined in (3.39). To implement the LCN discretization of the $\mathbf{K}$ operator the following expression needs to be computed:

$$
\mathbf{t}_{q_{m}}^{i} \cdot \mathbf{K}(\mathbf{J})=\mathbf{t}_{q_{m}}^{i} \cdot \sum_{n=1}^{N_{c}} \int_{\Omega_{n}} \overline{\mathbf{k}}\left(\mathbf{r}_{q_{m}}^{i}, \mathbf{r}^{\prime}\right) \cdot \mathbf{J}\left(\mathbf{r}^{\prime}\right) d \Omega^{\prime},
$$

where, $\mathbf{r}_{q_{m}}^{i}$ are the test points on $\Omega_{m}$. For $\Omega_{m}$ and $\Omega_{n}$ far from each other based on (3.42) a single point evaluation of the kernel is sufficient to calculate (3.81):

$$
\mathbf{t}_{q_{m}}^{i} \cdot \mathbf{K}(\mathbf{J})=\sum_{n=1}^{N_{c}} \sum_{j=1}^{N_{j}} \sum_{q=1}^{N_{q}} \mathbf{j}^{j}\left(\mathbf{r}_{q_{n}}^{j}\right) \omega_{q_{m}}^{i} \omega_{q_{n}}^{j} \mathbf{t}_{q_{m}}^{i} \cdot \overline{\mathbf{k}}\left(\mathbf{r}_{q_{m}}^{i}, \mathbf{r}_{q_{n}}^{j}\right) \cdot \mathbf{a}_{j}\left(\mathbf{r}_{q_{n}}^{j}\right),
$$

where, $\mathrm{j}^{j}\left(\mathbf{r}_{q_{n}}^{j}\right)$ were defined in (3.41). For near source and test cells the kernel for the $\mathbf{K}$ operator is singular. Consequently, based on (3.44) we need to compute the following integration to implement the local corrections:

$$
\begin{aligned}
\mathbf{t}_{q_{m}}^{i} \cdot \int_{\Omega_{n}} \overline{\mathbf{k}}\left(\mathbf{r}_{q_{m}}^{i}, \mathbf{r}^{\prime}\right) \cdot \mathbf{F}_{n}^{k, j}\left(\mathbf{r}^{\prime}\right) d \Omega^{\prime}= \\
\left.\mathbf{t}_{q_{m}}^{i} \cdot \int_{\Omega_{n}}\left(\nabla G\left(\mathbf{r}_{q_{m}}^{i}, \mathbf{r}^{\prime}\right) \times \mathbf{F}_{n}^{k, j}\left(\mathbf{r}^{\prime}\right)\right)\right|_{\mathbf{r}=\mathbf{r}_{q_{m}}^{i}} d \Omega^{\prime} .
\end{aligned}
$$


$\nabla G\left(\mathbf{r}, \mathbf{r}^{\prime}\right)$ in (3.80) is defined as:

$$
\nabla G\left(\mathbf{r}, \mathbf{r}^{\prime}\right)=\hat{\mathbf{R}} \frac{\partial G(R)}{\partial R}
$$

where, $\hat{\mathbf{R}}=\left(\mathbf{r}-\mathbf{r}^{\prime}\right) / \mathbf{r}-\mathbf{r}^{\prime} \mid$ and $R=\left|\mathbf{r}-\mathbf{r}^{\prime}\right| . \frac{\partial G(R)}{\partial R}$ is expressed as:

$$
\frac{\partial G(R)}{\partial R}=\frac{1}{4 \pi}\left(\frac{-j k R-1}{R^{2}}\right) \exp \left(-j k_{1} R\right)
$$

Consequently, (3.83) is rewritten as:

$$
\begin{aligned}
\mathbf{t}_{q_{m}}^{i} \cdot \int_{\Omega_{n}} \overline{\mathbf{k}}\left(\mathbf{r}_{q_{m}}^{i}, \mathbf{r}^{\prime}\right) \cdot \mathbf{F}_{n}^{k, j}\left(\mathbf{r}^{\prime}\right) d \Omega^{\prime}= & \\
& \left.\left.\int_{\Omega_{n}} \mathbf{t}_{q_{m}}^{i} \cdot\left(\hat{\mathbf{R}} \times \mathbf{F}_{n}^{k, j}\left(\mathbf{r}^{\prime}\right)\right)\right|_{\mathbf{r}=\mathbf{r}_{q_{m}}^{i}} \frac{\partial G(R)}{\partial R}\right|_{\mathbf{r}=\mathbf{r}_{q_{m}}^{i}} d \Omega^{\prime} .
\end{aligned}
$$

$\frac{\partial G(R)}{\partial R}$ has a $1 / R^{2}$ singularity when $\Omega_{n}$ is a surface. However, in the limit $\mathbf{r} \rightarrow \mathbf{r}^{\prime}, \hat{\mathbf{R}}$ becomes tangential to surface $\Omega_{n}$. Since $\mathbf{F}_{n}^{k, j}\left(\mathbf{r}^{\prime}\right)$ is in unitary vector direction which is tangential to $\Omega_{n}, \hat{\mathbf{R}} \times \mathbf{F}_{n}^{k, j}\left(\mathbf{r}^{\prime}\right)$ is normal to $\Omega_{n}$. $\mathbf{t}\left(\mathbf{r}_{q_{m}}^{i}\right)$ is tangential to $\Omega_{n}$. Therefore:

$$
\lim _{\mathbf{r} \rightarrow \mathbf{r}^{\prime}}\left(\left.\mathbf{t}_{q_{m}}^{i} \cdot\left(\hat{\mathbf{R}} \times \mathbf{F}_{n}^{k, j}\left(\mathbf{r}^{\prime}\right)\right)\right|_{\mathbf{r}=\mathbf{r}_{q_{m}}^{i}}\right)=0
$$

This zero reduces the order of the singularity of the integrand in (3.86) to $1 / R$. The integration is then computable by adaptive integration methods. Similarly, when $\Omega_{n}$ is a volume the order of singularity for (3.86) reduces to $1 / R^{2}$ which is an integrable singularity for volumes. 


\subsection{The Basis Functions for Local Corrections}

Considering the basis functions $\mathbf{F}_{n}^{k, j}(\mathbf{r})$ defined in (3.43), the current $\mathbf{J}(\mathbf{r})$ on $\Omega_{n}$ is implicitly approximated through the local corrections as:

$$
\mathbf{J}(\mathbf{r})=\sum_{k=1}^{N_{q}} \sum_{j=1}^{N_{j}} \beta_{n}^{k, j} \mathbf{F}_{n}^{k, j}(\mathbf{r})
$$

where, $N_{j}=2$ for a surface cell and $N_{j}=3$ for a volume cell and $\beta_{n}^{k, j}$ are the coefficients of the basis functions. Consequently, a polynomial interpolation of order $p$ is applied, wherein the abscissa for the corresponding quadrature rule are applied as the LCN test and source points. The Legendre polynomials are commonly applied as the interpolating basis functions for the local corrections, because they result in wellconditioned local correction matrices. Since the basis functions are confined to one cell, the currents are normally discontinuous across the boundaries of the mesh elements.

Two types of LCN basis functions developed based on Legendre polynomials are polynomial complete and mixed-order basis functions. The $p$ th order polynomial complete basis functions with support over the quadrilateral cell $\Omega_{n}$ are defined as:

$$
\mathbf{F}_{n}^{k, j}=\frac{P_{m}\left(u^{1}\right) P_{l}\left(u^{2}\right)}{\sqrt{g_{s}}} \mathbf{a}_{j}
$$

where, $\left(u^{1}, u^{2}\right)$ are local curvilinear coordinates on $\Omega_{n}, j=1,2$, and $m, l \in(0, p) ; \sqrt{g_{s}}$ is the surface Jacobian, and $\mathbf{a}_{j}$ are the unitary vectors. $P_{m}$ is the $m$ th order Legendre polynomial. Therefore, for a set of order $p$ polynomial complete basis functions over a quadrilateral cell (3.89) defines $2 N_{q}$ basis functions that are complete to order $p$ along $u^{1}$ and $u^{2}$ where, $N_{q}=(p+1)^{2}$ in this case. 
The pth order polynomial complete basis functions over a hexahedral cell $\Omega_{n}$ are similarly defined as:

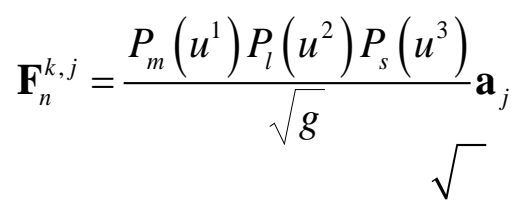

where, $\left(u^{1}, u^{2}, u^{3}\right)$ are local curvilinear coordinates on $\Omega_{n}, j=1,2,3$, and $m, l, s \in(0, p)$; $\sqrt{g}$ is the volume Jacobian, and $\mathbf{a}_{j}$ are the unitary vectors. $P_{m}$ is the Legendre polynomial of order $m$. Therefore, in this case (3.90) defines $3 N_{q}$ basis functions that are complete to order $p$ along $u^{1}, u^{2}$, and $u^{3}$ where, $N_{q}=(p+1)^{3}$.

As it is observed from (3.72), the LCN implementation of the $\mathbf{L}$ operator includes computation of the divergence of the basis functions. According to the continuity equation (2.75), the divergence of current is proportional to charge. The divergence of the polynomial complete basis functions are a set of basis functions that are incomplete along the curvilinear coordinates. Consequently, applying the polynomial complete basis functions for the current results in a set of incomplete basis functions interpolating the charge density. It has been shown that representation of the charge by an incomplete polynomial expansion specifically for geometries with sharp edges results in spurious solutions. To remedy this shortcoming of the polynomial complete basis functions, the mixed order basis functions were introduced [24].

The $p$ th mixed order basis functions with support over the quadrilateral cell $\Omega_{n}$ are defined as:

$$
\mathbf{F}_{n}^{k, 1}=\frac{P_{m}\left(u^{1}\right) P_{l}\left(u^{2}\right)}{\sqrt{g_{s}}} \mathbf{a}_{1}, \quad(m=0, \ldots, p+1 ; l=0, \ldots, p)
$$




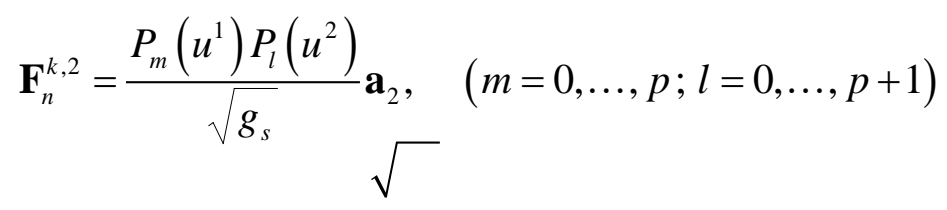

Therefore, $\mathbf{F}_{n}^{k, 1}$ is complete to order $p+1$ along $u^{1}$ and complete to order $p$ along $u^{2}$; $\mathbf{F}_{n}^{k, 2}$ is complete to order $p$ along $u^{1}$ and complete to order $p+1$ along $u^{2}$. Thus, the pth mixed order basis functions over a quadrilateral cell are comprised of $2 N_{q}$ vector bases where $N_{q}=(p+1)(p+2)$ in this case. According to (3.21), the divergence of $\mathbf{F}_{n}^{k, j}$ is expressed as:

$$
\nabla \cdot \mathbf{F}_{n}^{k, j}=\frac{1}{\sqrt{g_{s}}} \frac{\partial}{\partial u^{j}}\left(\sqrt{g_{s}} \mathbf{a}^{j} \cdot \mathbf{F}_{n}^{k, j}\right) .
$$

Thus, we obtain:

$$
\begin{aligned}
& \nabla \cdot \mathbf{F}_{n}^{k, 1}=\frac{1}{\sqrt{g_{s}}} \frac{\partial P_{m}\left(u^{1}\right)}{\partial u^{1}} P_{l}\left(u^{2}\right) \\
& \nabla \cdot \mathbf{F}_{n}^{k, 2}=\frac{1}{\sqrt{g_{s}}} P_{m}\left(u^{1}\right) \frac{\partial P_{l}\left(u^{2}\right)}{\partial u^{2}} .
\end{aligned}
$$

If the polynomial complete basis functions are used, $\nabla \cdot \mathbf{F}_{n}^{k, 1}$ is complete to order $p-1$ along $u^{1}$ and complete to order $p$ along $u^{2} ; \nabla \cdot \mathbf{F}_{n}^{k, 2}$ is complete to order $p$ along $u^{1}$ and complete to order $p-1$ along $u^{2}$. However, it is observed that by applying the mixed-order basis functions defined in (3.91) and (3.92) $\nabla \cdot \mathbf{F}_{n}^{k, j}$ is complete to order $p$ along $u^{1}$ and $u^{2}$. It has been verified that the application of mixed-order basis functions improves the accuracy of the LCN method solution and lowers the condition number of the system matrix [24]. Similarly, the pth mixed-order basis functions over a hexahedral cell are defined as: 


$$
\begin{aligned}
& \mathbf{F}_{n}^{k, 1}=\frac{P_{m}\left(u^{1}\right) P_{l}\left(u^{2}\right) P_{s}\left(u^{3}\right)}{\sqrt{g}} \mathbf{a}_{1}, \quad(m=0, \ldots, p+1 ; l=0, \ldots, p ; s=0, \ldots, p) \\
& \mathbf{F}_{n}^{k, 2}=\frac{P_{m}\left(u^{1}\right) P_{l}\left(u^{2}\right) P_{s}\left(u^{3}\right)}{\sqrt{g}} \mathbf{a}_{2}, \quad(m=0, \ldots, p ; l=0, \ldots, p+1 ; s=0, \ldots, p) \\
& \sqrt{ } \\
& \mathbf{F}_{n}^{k, 3}=\frac{P_{m}\left(u^{1}\right) P_{l}\left(u^{2}\right) P_{s}\left(u^{3}\right)}{\sqrt{g}} \mathbf{a}_{3}, \quad(m=0, \ldots, p ; l=0, \ldots, p ; s=0, \ldots, p+1)
\end{aligned}
$$

Therefore, the mixed-order LCN discretization with charge complete to order $p$ incorporates $3 N_{q}$ basis functions over a hexahedral cell where, $N_{q}=(p+1)^{2}(p+2)$.

\subsection{LCN Test and Source Scheme}

As it was noted before unlike the element based MOM wherein the DOFs include the coefficients of the basis functions assigned to each mesh element, the DOFs of the point based LCN method are comprised of the current samples at the abscissa of the quadrature rule corresponding to the local correction basis functions. For an order-p polynomial complete set of local correction basis functions on a quadrilateral cell the unknown current samples are located on a $Q^{u 1}(p+1) \times Q^{u 2}(p+1)$ quadrature sample grid in which $Q^{u j}(p+1)$ indicates a $(p+1)$ point quadrature rule along $u^{j}$. With respect to (3.39), at each point $\mathbf{r}_{q_{n}}^{j}$ on the grid there exist two unknown current samples $\mathrm{J}^{j}\left(\mathbf{r}_{q_{n}}^{j}\right), j=1,2$, in directions $\mathbf{a}_{1}$ and $\mathbf{a}_{2}$. It should be noted that for polynomial complete basis functions $\mathbf{r}_{q_{n}}^{1}$ and $\mathbf{r}_{q_{n}}^{2}$ are overlapping each other. The quadrature grid is also used for the test points $\mathbf{r}_{q_{m}}^{i}$. Two test vectors $\mathbf{t}_{q_{m}}^{i}, i=1,2$, are assigned to each point of the order complete quadrature sample grid [45]. 
Similarly, for an order-p polynomial complete set of local correction basis functions on a hexahedral cell the unknown current samples are located on a $Q^{u 1}(p+1) \times Q^{u 2}(p+1) \times Q^{u 3}(p+1)$ quadrature sample grid. There are three current samples in directions $\mathbf{a}_{1}, \mathbf{a}_{2}$, and $\mathbf{a}_{3}$ at each point on the grid. Three test vectors $\mathbf{t}_{q_{m}}^{i}, i=1,2,3$, are assigned to each point of the order complete quadrature sample grid.

For a set of mixed-order basis functions with charge complete to order $p$ on a quadrilateral cell, the unknown current samples $\mathrm{J}^{1}\left(\mathbf{r}_{q_{n}}^{1}\right)$ in direction $\mathbf{a}_{1}$ are located on a $Q^{u 1}(p+2) \times Q^{u 2}(p+1)$ quadrature sample grid, and the unknown current samples $\mathrm{J}^{2}\left(\mathbf{r}_{q_{n}}^{2}\right)$ in direction $\mathbf{a}_{2}$ are located on a $Q^{u 1}(p+1) \times Q^{u 2}(p+2)$ quadrature sample grid. The appropriate test vectors $\mathbf{t}_{q_{m}}^{1}$ are assigned to the $Q^{u 1}(p+2) \times Q^{u 2}(p+1)$ quadrature sample grid; the test vectors $\mathbf{t}_{q_{m}}^{2}$ are assigned to the $Q^{u 1}(p+1) \times Q^{u 2}(p+2)$ quadrature sample grid.

Similarly, for a hexahedral cell the current samples in direction $\mathbf{a}_{1}$ and the test vectors $\mathbf{t}_{q_{m}}^{1}$ are assigned to a $Q^{u 1}(p+2) \times Q^{u 2}(p+1) \times Q^{u 3}(p+1)$ quadrature sample grid; The current samples in direction $\mathbf{a}_{2}$ and the test vectors $\mathbf{t}_{q_{m}}^{2}$ are assigned to a $Q^{u 1}(p+1) \times Q^{u 2}(p+2) \times Q^{u 3}(p+1)$ quadrature sample grid; The current samples in direction $\mathbf{a}_{3}$ and the test vectors $\mathbf{t}_{q_{m}}^{3}$ are assigned to a $Q^{u 1}(p+1) \times Q^{u 2}(p+1) \times Q^{u 3}(p+2)$ quadrature sample grid. 


\subsection{Selecting LCN Test Vectors}

As it was mentioned in the previous section, in implementing the LCN discretization the test vectors are assigned to each quadrature point in the quadrature sample grid and dot product of the test vector and the integral operator is computed at each test point. Consequently, the test vectors have to be selected in such a way that the integral operator is sampled appropriately. It has been shown that the unitary vectors are a proper choice to test the $\mathbf{L}$ operator, i.e.:

$$
\mathbf{t}_{q_{m}}^{i}=\mathbf{a}_{i}\left(r_{q_{m}}^{i}\right)
$$

However, for integral equations with block-diagonal terms such as MFIE in (2.55) and VIE in (2.61) wherein the LCN discretization of the block diagonal terms includes calculation of $\mathbf{t}_{q_{m}}^{i} \cdot \mathrm{J}^{j}\left(\mathbf{r}_{q_{n}}^{j}\right)$, the reciprocal unitary vectors scaled by the Jacobian are the appropriate choice since they diagonalize this operator [23]. Therefore, we have:

$$
\mathbf{t}_{q_{m}}^{i}=\sqrt{g_{q_{m}}^{i}} \mathbf{a}^{i}\left(r_{q_{m}}^{i}\right)
$$

where, $\sqrt{g_{q_{m}}^{i}}$ is the Jacobian at $\mathbf{r}_{q_{m}}^{i}$. 


\section{Chapter 4. THE Augmented VIE Formulation}

As discussed in chapter 2, the volume integral equation (VIE) provides a powerful approach for solving radiation and scattering problems involving nonlinear and/or inhomogeneous materials. The VIE formulations (2.61) and (2.62) are of the form of an integral operator plus the identity operator scaled by the inverse of the material parameters of the scatterer and the operating frequency. By applying the LCN discretization of the VIE the material properties of the scatterer become confined to the diagonal of the system matrix. Therefore, by increasing the material contrasts the condition number of the VIE system matrix tends to increase [32]. Specifically at low frequencies, the condition number of the VIE system matrix grows linearly by increasing the material contrast. Previously, a Helmholtz decomposition based preconditioner has been introduced to address this problem [47]. In the following, an alternative approach called augmented VIE (AVIE) is introduced which utilizes the surface and volume charges as additional DOFs and incorporates the current continuity and charge neutrality constraints [33]. It is shown that the condition number of the system matrix associated with the AVIE formulation at low frequencies is independent of the material parameters of the scatterer.

\subsection{Development of the AVIE Formulation}

In the rest of this chapter, we consider scattering from pure dielectric materials. The formulations for pure magnetic materials can be obtained by using the duality relationships [1]. As considered in chapter 2, the VIE formulation for scattering from an inhomogeneous dielectric material located in free space is described as: 


$$
\begin{aligned}
\mathbf{t} \cdot \mathbf{E}^{i n c}(\mathbf{r}) & =\frac{\mathbf{t} \cdot \mathbf{J}_{v}(\mathbf{r})}{j \omega\left(\varepsilon_{2}(\mathbf{r})-\varepsilon_{0}\right)}+j k_{0} \eta_{0} \mathbf{t} \cdot \int_{V} \mathbf{J}_{v}\left(\mathbf{r}^{\prime}\right) G\left(\mathbf{r}, \mathbf{r}^{\prime}\right) d v^{\prime} \\
& +j k_{0}^{-1} \eta_{0} \mathbf{t} \cdot \nabla \nabla \cdot \int_{V} \mathbf{J}_{v}\left(\mathbf{r}^{\prime}\right) G\left(\mathbf{r}, \mathbf{r}^{\prime}\right) d v^{\prime}
\end{aligned}
$$

where, $\mathbf{J}_{v}$ is the volume current density, and $G\left(\mathbf{r}, \mathbf{r}^{\prime}\right)$ is the free space Green's function. $k_{0}$ and $\eta_{0}$ are respectively the wave number and the characteristic impedance of the free space. $V$ indicates the volume of the scatterer. Using the vector identities, the above equation can be expressed as:

$$
\begin{aligned}
\mathbf{t} \cdot \mathbf{E}^{i n c}(\mathbf{r}) & =\frac{\mathbf{t} \cdot \mathbf{J}_{v}(\mathbf{r})}{j \omega\left(\varepsilon_{2}(\mathbf{r})-\varepsilon_{0}\right)}+j k_{0} \eta_{0} \mathbf{t} \cdot \int_{V} \mathbf{J}_{v}\left(\mathbf{r}^{\prime}\right) G\left(\mathbf{r}, \mathbf{r}^{\prime}\right) d v^{\prime} \\
& +j k_{0}{ }^{-1} \eta_{0} \mathbf{t} \cdot \nabla \int_{V}\left(\nabla^{\prime} \cdot \mathbf{J}_{v}\left(\mathbf{r}^{\prime}\right)\right) G\left(\mathbf{r}, \mathbf{r}^{\prime}\right) d v^{\prime},
\end{aligned}
$$

In the following numerical results, the volume $V$ is discretized into curvilinear hexahedral cells. The currents inside the volume cells are continuous. As it was discussed in the LCN discretization of the $\mathbf{L}$ operator in chapter 3, the integral in (4.2) thus can be divided into integrals over volume cells. Similar to (3.72) by the application of the divergence theorem and noting that the currents are not continuous across the interfaces of the volume cells, (4.2) can be expressed as:

$$
\begin{aligned}
\mathbf{t} \cdot \mathbf{E}^{\text {inc }}(\mathbf{r})=\mathbf{t} \cdot \sum_{\text {cells }} \frac{\mathbf{J}_{v}(\mathbf{r})}{j \omega\left(\varepsilon_{2}(\mathbf{r})-\varepsilon_{0}\right)} & +j k_{0} \eta_{0} \mathbf{t} \cdot \sum_{\text {cells }} \int_{V_{c}} \mathbf{J}_{v}\left(\mathbf{r}^{\prime}\right) G\left(\mathbf{r}, \mathbf{r}^{\prime}\right) d v^{\prime} \\
& +j k_{0}^{-1} \eta_{0} \sum_{\text {cells }} \int_{V_{c}}\left(\mathbf{t} \cdot \nabla G\left(\mathbf{r}, \mathbf{r}^{\prime}\right)\right)\left[\nabla^{\prime} . \mathbf{J}_{v}\left(\mathbf{r}^{\prime}\right)\right] d v^{\prime} \\
& -j k_{0}^{-1} \eta_{0} \sum_{\text {cells }} \int_{\partial V_{c}} \mathbf{n}_{c}^{\prime} \cdot \mathbf{J}_{v}\left(\mathbf{r}^{\prime}\right)\left(\mathbf{t} \cdot \nabla G\left(\mathbf{r}, \mathbf{r}^{\prime}\right)\right) d s^{\prime},
\end{aligned}
$$

where, $V_{c}$ is the volume associated with the individual cell. $\partial V_{c}$ are the face interfaces bounding a volume cell. $\mathbf{n}_{c}^{\prime}$ is the outward normal to the $\partial V_{c}$ at source points. The last 
term arises from the current discontinuities across the surfaces bounding the volume cells and is represented as sum of the contributions of the current discontinuities across the surfaces of the mesh:

$$
-j k_{0}^{-1} \eta_{0} \sum_{\text {faces }} \int_{S_{c}}\left(\mathbf{n}_{c_{1}}^{\prime} \cdot \mathbf{J}_{v_{1}}\left(\mathbf{r}^{\prime}\right)+\mathbf{n}_{c_{2}}^{\prime} \cdot \mathbf{J}_{v_{2}}\left(\mathbf{r}^{\prime}\right)\right)\left(\mathbf{t} \cdot \nabla G\left(\mathbf{r}, \mathbf{r}^{\prime}\right)\right) d s^{\prime},
$$

where, $S_{c}$ is a mesh face. $\mathbf{n}_{c_{1}}^{\prime}$ and $\mathbf{n}_{c_{2}}^{\prime}$ are the normal vectors to the face corresponding to the volume cells sharing the face. $\mathbf{J}_{v_{1}}$ and $\mathbf{J}_{v_{2}}$ are associated with the current samples on the two cells sharing the face. The continuity equation on a volume cell is expressed as:

$$
\nabla \cdot \mathbf{J}_{v}(\mathbf{r})=-j \omega q_{v}(\mathbf{r})
$$

where, $q_{v}(\mathbf{r})$ is the volume charge density. The continuity equation on the faces of the mesh is represented as:

$$
-\mathbf{n}_{c_{1}} \cdot \mathbf{J}_{v_{1}}(\mathbf{r})-\mathbf{n}_{c_{2}} \cdot \mathbf{J}_{v_{2}}(\mathbf{r})=-j \omega q_{s}(\mathbf{r})
$$

where, $q_{s}(\mathbf{r})$ is the surface charge density. Substituting (4.4) to (4.6) in (4.3) yields:

$$
\begin{aligned}
\mathbf{t} \cdot \mathbf{E}^{\text {inc }}(\mathbf{r})=\mathbf{t} \cdot \sum_{\text {cells }} \frac{\mathbf{J}_{v}(\mathbf{r})}{j \omega\left(\varepsilon_{2}(\mathbf{r})-\varepsilon_{0}\right)} & +\eta_{0}\left[\mathbf{t} \cdot \sum_{\text {cells }} \int_{V_{c}} j k_{0} \mathbf{J}_{v}\left(\mathbf{r}^{\prime}\right) G\left(\mathbf{r}, \mathbf{r}^{\prime}\right) d v^{\prime}\right. \\
& +\sum_{\text {cells } V_{V_{c}}}\left(\mathbf{t} \cdot \nabla G\left(\mathbf{r}, \mathbf{r}^{\prime}\right)\right) c_{0} q_{v}\left(\mathbf{r}^{\prime}\right) d v^{\prime} \\
& \left.+\sum_{\text {faces }} \int_{S_{c}}\left(\mathbf{t} \cdot \nabla G\left(\mathbf{r}, \mathbf{r}^{\prime}\right)\right) c_{0} q_{s}\left(\mathbf{r}^{\prime}\right) d s^{\prime}\right],
\end{aligned}
$$

where, $c_{0}$ is the speed of light. By utilizing $\frac{\mathbf{J}_{v}}{j k_{0}}, c_{0} q_{v}$, and $c_{0} q_{s}$ as unknowns the matrix form of the AVIE comprising (4.5), (4.6), and (4.7) is: 


$$
\left[\begin{array}{ccc}
\mathbf{L}_{A} & \mathbf{L}_{q_{v}} & \mathbf{L}_{q_{s}} \\
\mathbf{D}_{v} & \mathbf{I} & \mathbf{0} \\
\mathbf{D}_{s} & \mathbf{0} & \mathbf{I}
\end{array}\right]\left[\begin{array}{c}
\overline{\mathbf{j}} \\
\overline{j k_{0}} \\
c_{0} \overline{\mathrm{q}}_{v} \\
c_{0} \overline{\mathrm{q}}_{s}
\end{array}\right]=\frac{1}{\eta_{0}}\left[\begin{array}{c}
\overline{\mathrm{f}} \\
\mathbf{0} \\
\mathbf{0} \\
\mathbf{0}
\end{array}\right],
$$

where $\bar{j}$ and $\bar{f}$ were defined in (3.55). $\bar{q}_{v}$ and $\bar{q}_{s}$ are defined in a similar way and include the unknown samples of volume and surface charges. It should be mentioned that a mixed order representation of current with charge complete to order $p$ has been applied. Therefore, the $p$ th order complete Legendre polynomial basis functions are used to represent the charge.

$$
\begin{gathered}
\mathbf{L}_{A}=\frac{1}{\left(\frac{\varepsilon_{d}}{\varepsilon}-1\right)} \mathbf{t} \cdot-k_{0}^{2} \mathbf{t} \cdot \mathbf{A}, \\
\mathbf{L}_{q_{v}}=\mathbf{L}_{q_{s}}=\mathbf{t} \cdot \nabla \Phi,
\end{gathered}
$$

In (4.9), the vector potential operator $\mathbf{A}$ is defined as:

$$
\mathbf{A}(\mathbf{J})=\int_{V} \mathbf{J}\left(\mathbf{r}^{\prime}\right) \mathbf{G}\left(\mathbf{r}, \mathbf{r}^{\prime}\right) d v^{\prime},
$$

and the operator $\Phi$ is the scalar potential operator defined as:

$$
\Phi(q)=\int_{\Omega} q\left(\mathbf{r}^{\prime}\right) \mathbf{G}\left(\mathbf{r}, \mathbf{r}^{\prime}\right) d \Omega^{\prime} .
$$

where $\Omega$ is the domain of $q . \mathbf{D}_{v}$ and $\mathbf{D}_{s}$ are sparse non-square matrices associated with continuity equations (4.5) and (4.6) respectively, and $\mathbf{I}$ is the identity matrix. As will be shown in the numerical results, the LCN discretization of the AVIE formulation in (4.8) provides stable condition numbers by increasing the material contrast at low frequencies. However, the condition number of the original system matrix in (4.8) is still high in some cases. However, it will be shown that the AVIE formulation provides sufficient flexibility 
to enable the resulting linear system to be appropriately scaled by the application of an iterative diagonal scaling algorithm in order to significantly improve the matrix condition numbers. It will also be observed that similar attempts to scale the regular VIE do not significantly improve the condition numbers of the corresponding system matrices.

\subsection{The Iterative Diagonal Scaling Algorithm}

The matrix balancing algorithms provide viable techniques in improving the condition number of large sparse matrices [48]. The $n \times n$ matrix $A$ is balanced in $\alpha$ norm if:

$$
\|A(i,:)\|_{\alpha}=\|A(:, i)\|_{\alpha},
$$

where, $i=1, \ldots, n$. $A(i,:)$ and $A(:, i)$ are respectively the $i$ th row and column of the matrix $A$.

Simultaneous left and right diagonal scaling of the system matrix has proven effective in matrix balancing [34]. In the following, we illustrate an iterative algorithm in order to find the left and right diagonal factors. Suppose the matrix equation resulting from the discretization of the problem formulation is described as:

$$
\mathbf{A} \mathbf{x}=\mathbf{B},
$$

and assume the system matrix after simultaneous left and right diagonal scaling is expressed as:

$$
\hat{\mathbf{A}} \hat{\mathbf{x}}=\hat{\mathbf{B}}
$$

Therefore, we can write:

$$
\begin{gathered}
\hat{\mathbf{A}}=\mathbf{D}_{L} \mathbf{A} \mathbf{D}_{R}, \\
\hat{\mathbf{x}}=\mathbf{D}_{R}^{-1} \mathbf{x},
\end{gathered}
$$




$$
\hat{\mathbf{B}}=\mathbf{D}_{L} \mathbf{B},
$$

where, $\mathbf{D}_{L}$ and $\mathbf{D}_{R}$ are left and right diagonal matrices which are computed by the following algorithm:

$$
\begin{aligned}
\hline \text { Initializing: } \operatorname{err}=1 ; \text { tol }=0.0001 ; k=0 ; r=\sqrt{n_{r} / n_{c}} ; \\
\mathbf{A}^{(0)}=\mathbf{A} ; \mathbf{D}_{L}^{(k)}=\mathbf{I} ; \mathbf{D}_{R}^{(k)}=\mathbf{I} ; \mathbf{d}_{L}^{(k)}=\mathbf{d}_{R}^{(k)}=\mathbf{0}
\end{aligned}
$$$$
\mathbf{A}^{(0)}=\mathbf{A} ; \mathbf{D}_{L}^{(k)}=\mathbf{I} ; \mathbf{D}_{R}^{(k)}=\mathbf{I} ; \mathbf{d}_{L}^{(k)}=\mathbf{d}_{R}^{(k)}=\mathbf{0}
$$

end

$$
\mathbf{D}_{L}=\mathbf{D}_{L}^{(k)} ; \quad \mathbf{D}_{R}=\mathbf{D}_{R}^{(k)} ;
$$

Algorithm 1: Iterative diagonal scaling algorithm 
where $n_{r}$ and $n_{c}$ are the number of rows and columns of the matrix $\mathbf{A}$. As it will be shown in the numerical results, the iterative scaling algorithm applied to the AVIE converges to the desired accuracy in less than 40 iterations for the studied test cases.

\subsection{Numerical Results}

In this section we consider the numerical results of the application of the AVIE formulation to different test cases. We start by investigating the scattering from a onemeter radius dielectric sphere illuminated by a $-\hat{\mathbf{z}}$ traveling plane wave polarized along the $\hat{\mathbf{x}}$ direction. In all the following examples, the AVIE and VIE system matrices are preconditioned with iterative scaling algorithm described in the previous section. The dielectric sphere is meshed by linear hexahedral volume cells. Figure 2 shows the condition number of the AVIE system matrix versus the relative permittivity of the dielectric sphere for different mesh sizes compared to the corresponding VIE results at 1Hz. A mixed order LCN discretization of the AVIE and VIE formulations with charge complete to order one has been applied. As it is observed the condition number of the AVIE system matrix is stable by increasing the relative permittivity, however the condition numbers of the VIE are increasing linearly. Figure 3 to Figure 6 show the convergence of the left and right errors $\operatorname{err}_{\mathbf{R}}$ and $\operatorname{err}_{\mathbf{L}}$ defined in the iterative scaling algorithm applied to the AVIE formulation for scattering from the one meter radius dielectric sphere at $1 \mathrm{~Hz}$ for different mesh sizes. Figure 3 and Figure 4 are related to the dielectric sphere with relative permittivity $\varepsilon_{r}=256$; Figure 5 and Figure 6 are related to the dielectric sphere with relative permittivity $\varepsilon_{r}=1000$. The figures show that as the mesh is refined the error plots are converging to each other. 
Figure 7 demonstrates the high order (HO) convergence of the RMS error in near electric and magnetic fields at $1 \mathrm{~Hz}$ versus basis order computed by the AVIE formulation applied to the $1 \mathrm{~m}$ radius dielectric sphere for different values of $\varepsilon_{r}$. The fields are computed on a semi-circle with a radius of 1.5 meters. The errors are calculated relative to the Mie series solution. The sphere is meshed by 32 sixth order curvilinear hexahedral cells, and the integration tolerances are $10^{-6}$.

Figure 8 shows the high order (HO) convergence of the error in radar cross section (RCS) computed by the AVIE for the $1 \mathrm{~m}$ radius dielectric sphere with $\varepsilon_{r}=16$ at $50 \mathrm{MHz}$. The sphere is meshed by 32 sixth order curvilinear hexahedral cell, and the integration tolerances are $10^{-6}$. The error is calculated relative to the Mie series solution.

Figure 9 demonstrates the error of near electric fields at $1 \mathrm{~Hz}$ versus relative permittivity for a $1 \mathrm{~m}$ radius dielectric sphere computed by AVIE and VIE formulations used in conjunction with LOGOS. The sphere is meshed by 1080 linear hexahedral cells. A mixed order LCN discretization of the AVIE and VIE formulations with charge complete to order one has been utilized. Errors are computed for fixed factorization and fill error tolerances, $10^{-3}$, and number of levels $l=3$ [8]. As it is observed the near electric fields computed by the AVIE are more accurate than those computed by the VIE. This occurs because the practical application of OL-LOGOS algorithm requires wellconditioned formulations and AVIE provides a better conditioned system matrix than the VIE.

Finally, to evaluate the performance of AVIE and VIE in conjunction with iterative dense solvers the generalized minimal residual (GMRES) method was applied to solve the scattering from the dielectric sphere. However, it was observed that the GMRES 
demonstrates a poor performance for the AVIE system of equations compared to the VIE. This occurs because the GMRES is sensitive to the eigenvalue pattern of the system matrix. It has been shown that GMRES is appropriate for the system matrices with a compact eigenvalue pattern wherein the closed boundary inclosing the eigenvalues does not encircle the center of coordinates. Figure 10 and Figure 11 respectively represent the eigenvalues of the preconditioned VIE and AVIE system matrices for scattering from the dielectric sphere with $\varepsilon_{r}=400$ at $1 \mathrm{~Hz}$ modeled by 256 linear hexahedral cells. A mixed order discretization of the formulation with charge complete to order zero has been utilized. As it is observed the eigenvalue pattern of the reconditioned VIE is more compact than that of the preconditioned AVIE. Moreover, the eigenvalue pattern of the AVIE surrounds the center of the coordinates. 


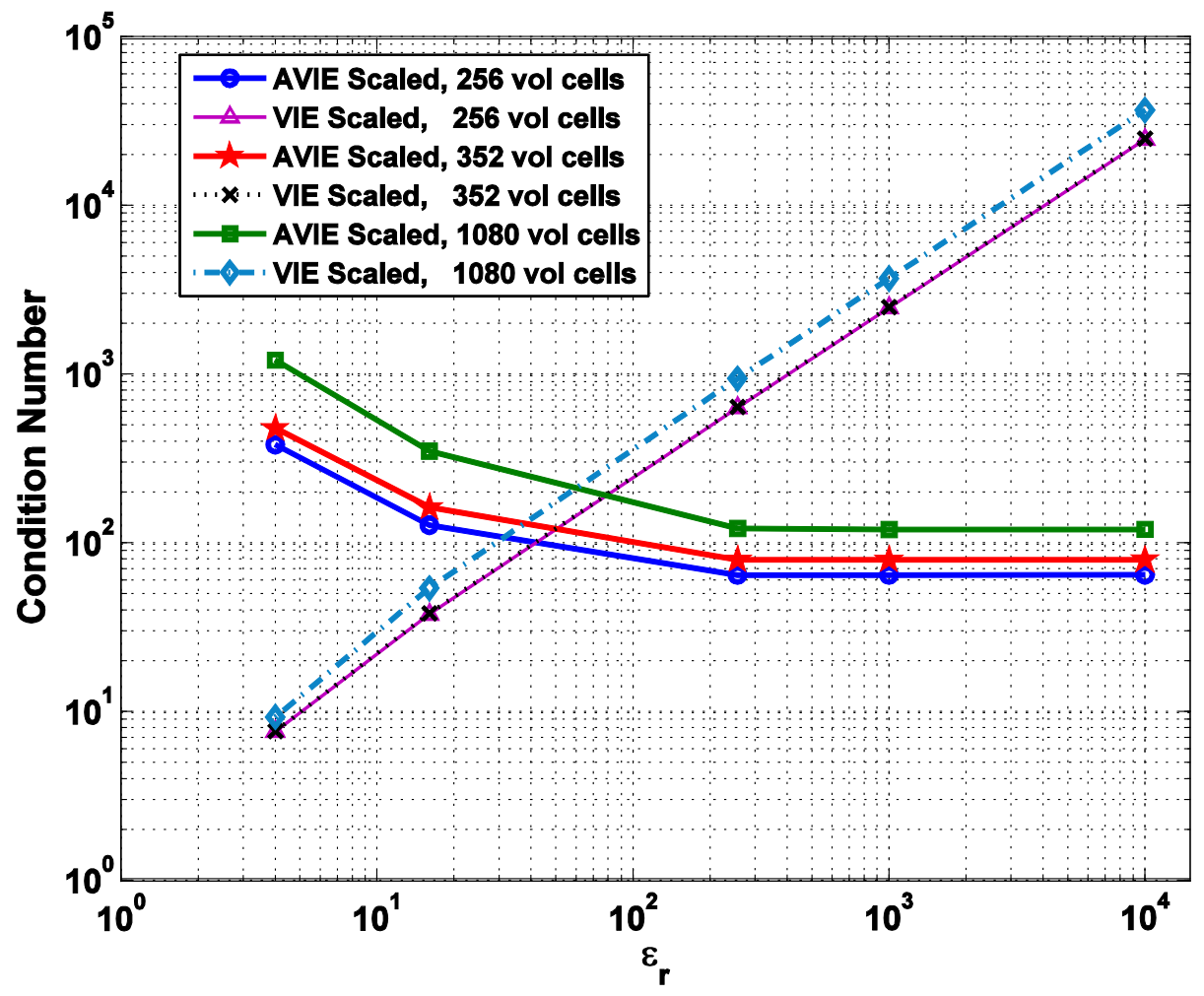

Figure 2: The condition numbers of the LCN discretization of the AVIE and VIE formulations with iterative scaling applied to $1 \mathrm{~m}$ radius dielectric sphere with different mesh sizes versus the relative permittivity at $1 \mathrm{~Hz}$ 


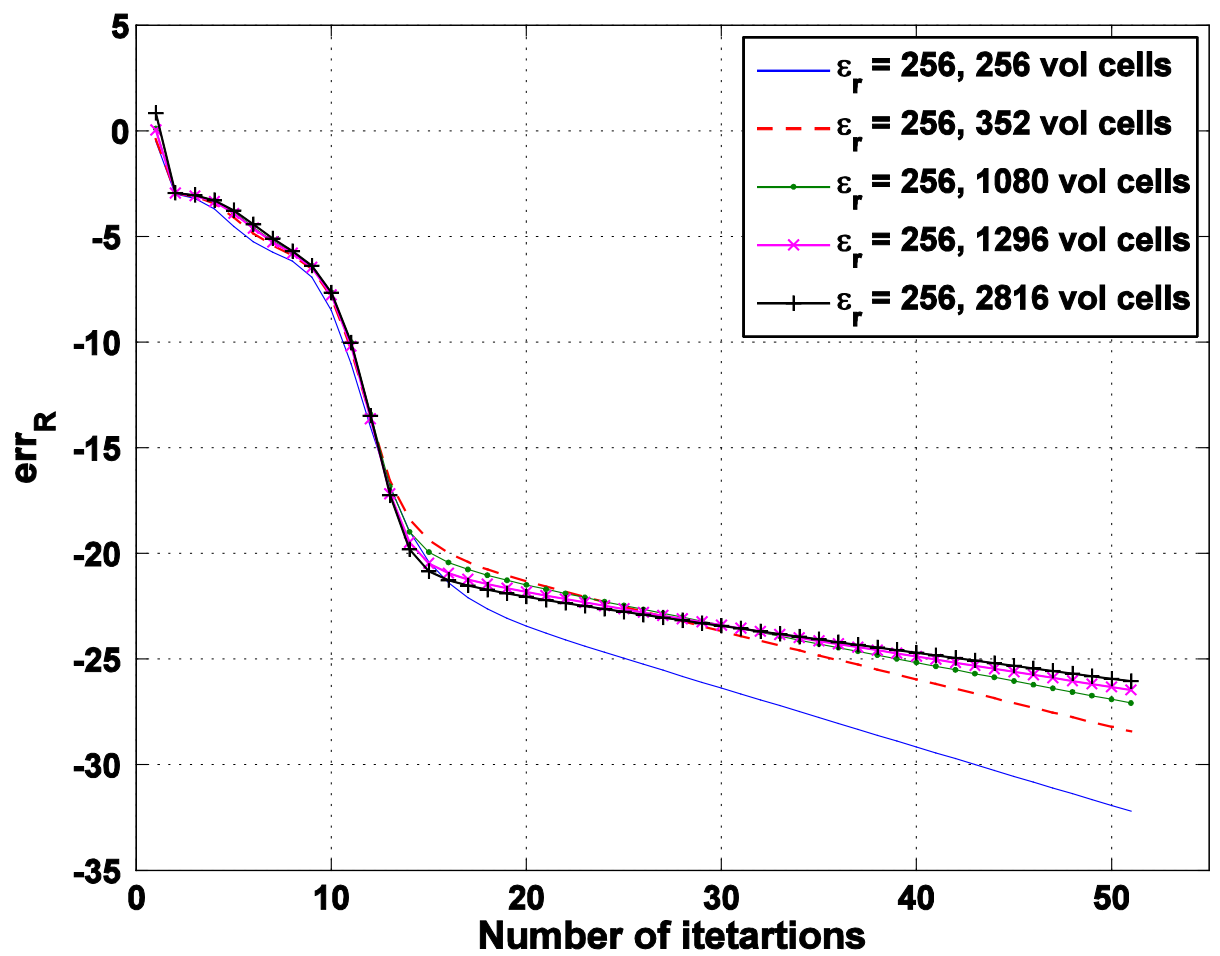

Figure 3: The convergence of the right errors $\operatorname{err}_{\mathbf{R}}$ defined in the iterative scaling algorithm applied to the AVIE formulation for scattering from the one meter radius dielectric sphere with $\varepsilon_{r}=256$ at $1 \mathrm{~Hz}$ 


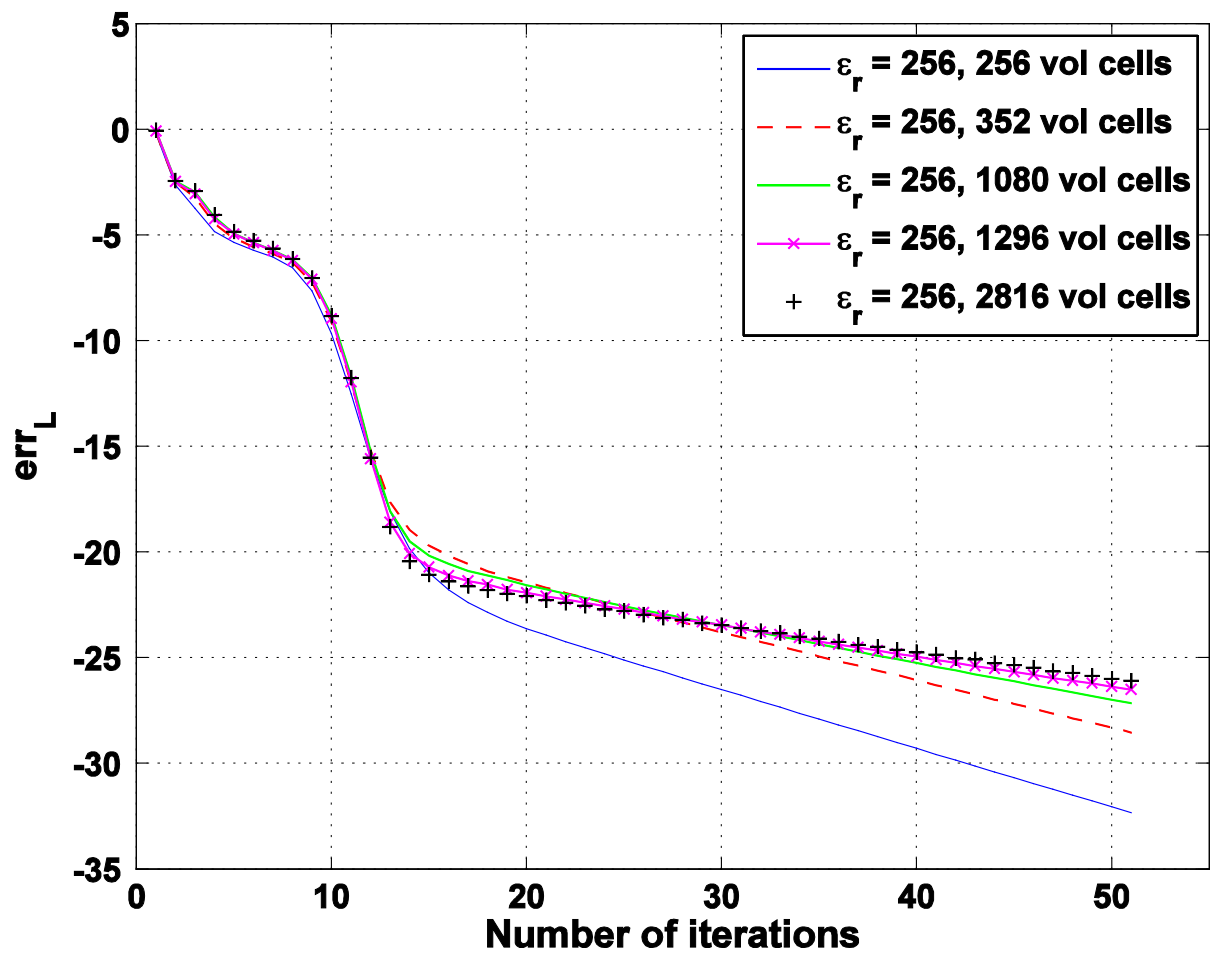

Figure 4: The convergence of the left errors $e r r_{L}$ defined in the iterative scaling algorithm applied to the AVIE formulation for scattering from the one meter radius dielectric sphere with $\varepsilon_{r}=256$ at $1 \mathrm{~Hz}$ 


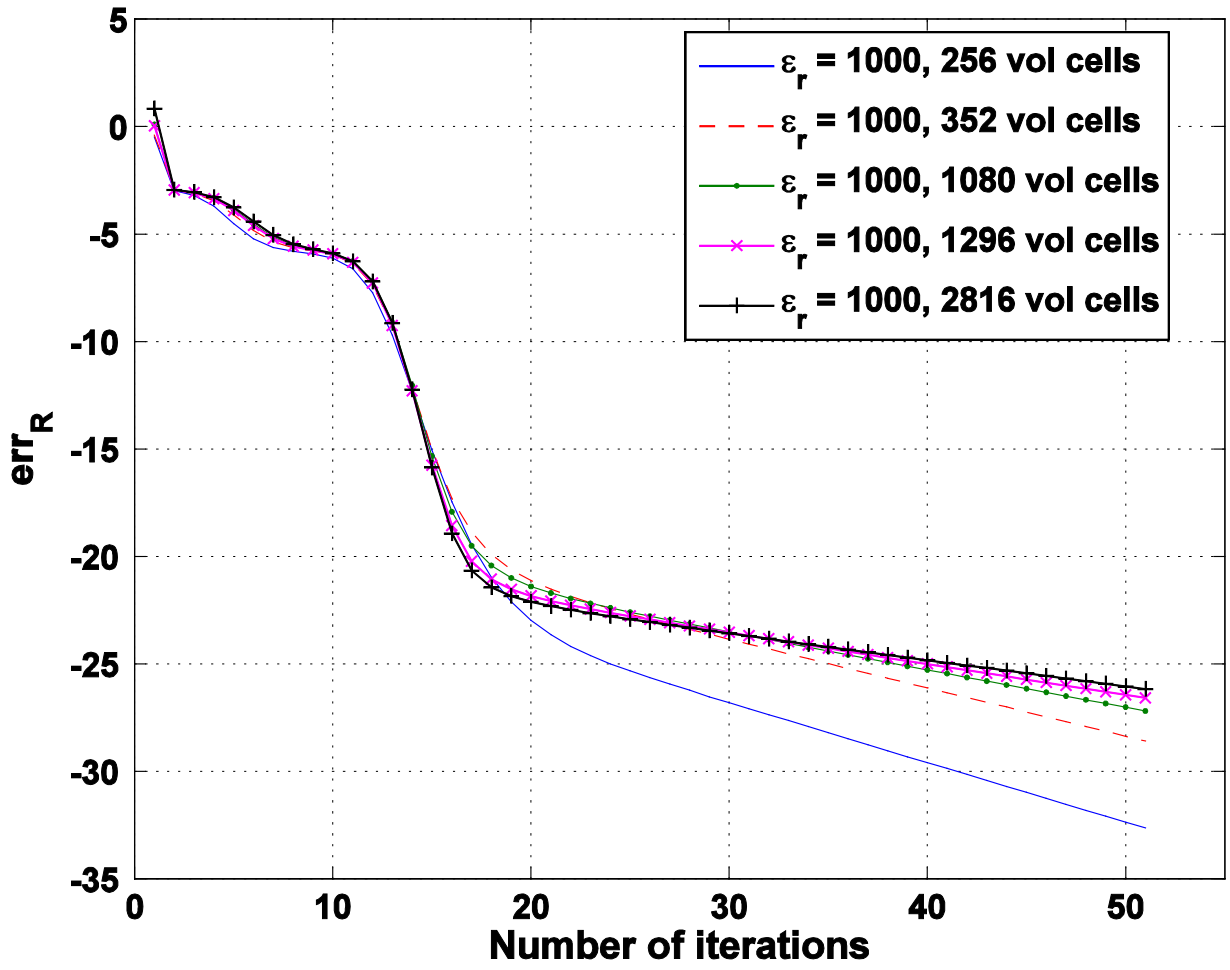

Figure 5: The convergence of the right errors $\operatorname{er~}_{\mathbf{R}}$ defined in the iterative scaling algorithm applied to the AVIE formulation for scattering from the one meter radius dielectric sphere with $\varepsilon_{r}=1000$ at $1 \mathrm{~Hz}$ 


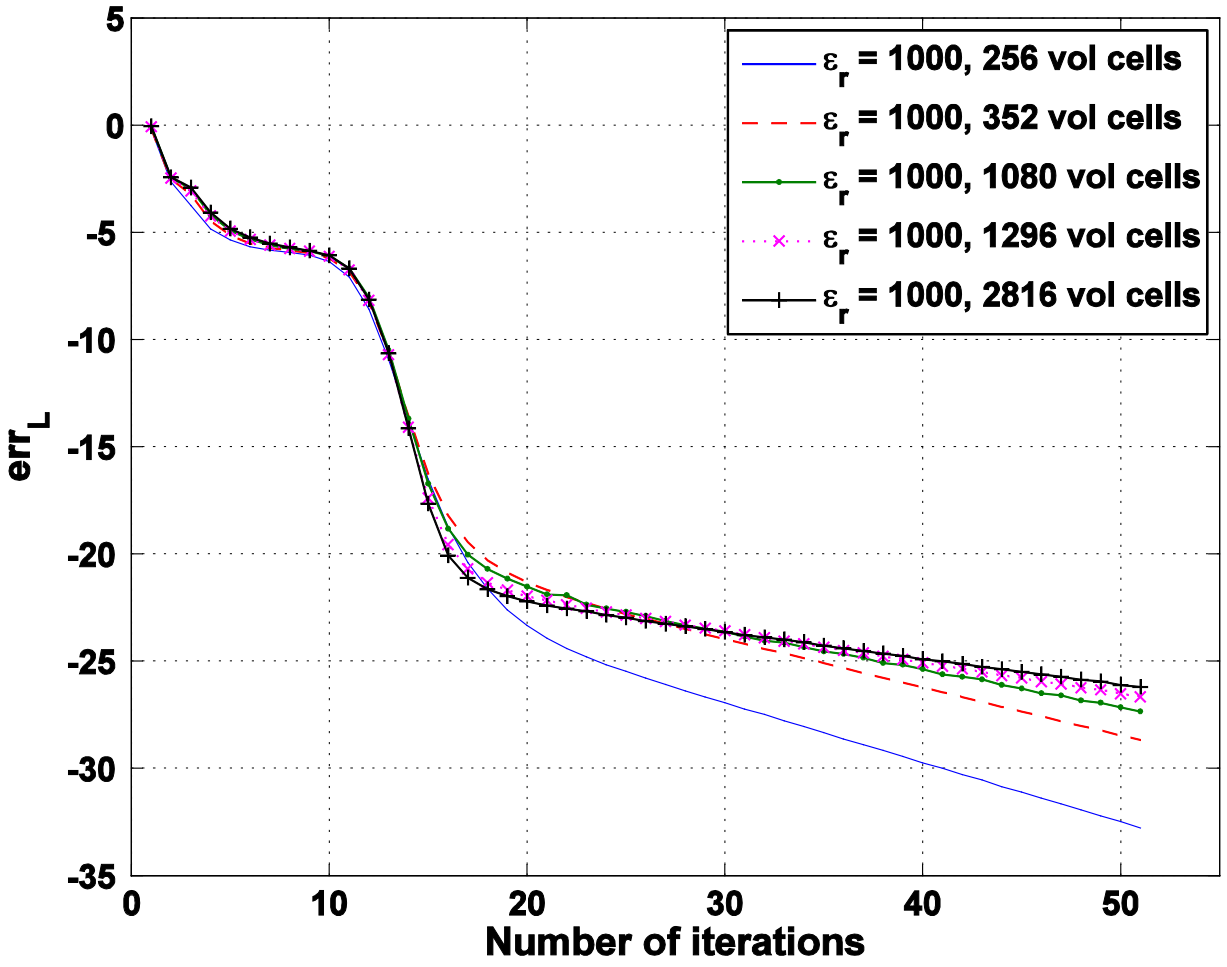

Figure 6: The convergence of the left errors $e r r_{L}$ defined in the iterative scaling algorithm applied to the AVIE formulation for scattering from the one meter radius dielectric sphere with $\varepsilon_{r}=1000$ at $1 \mathrm{~Hz}$ 


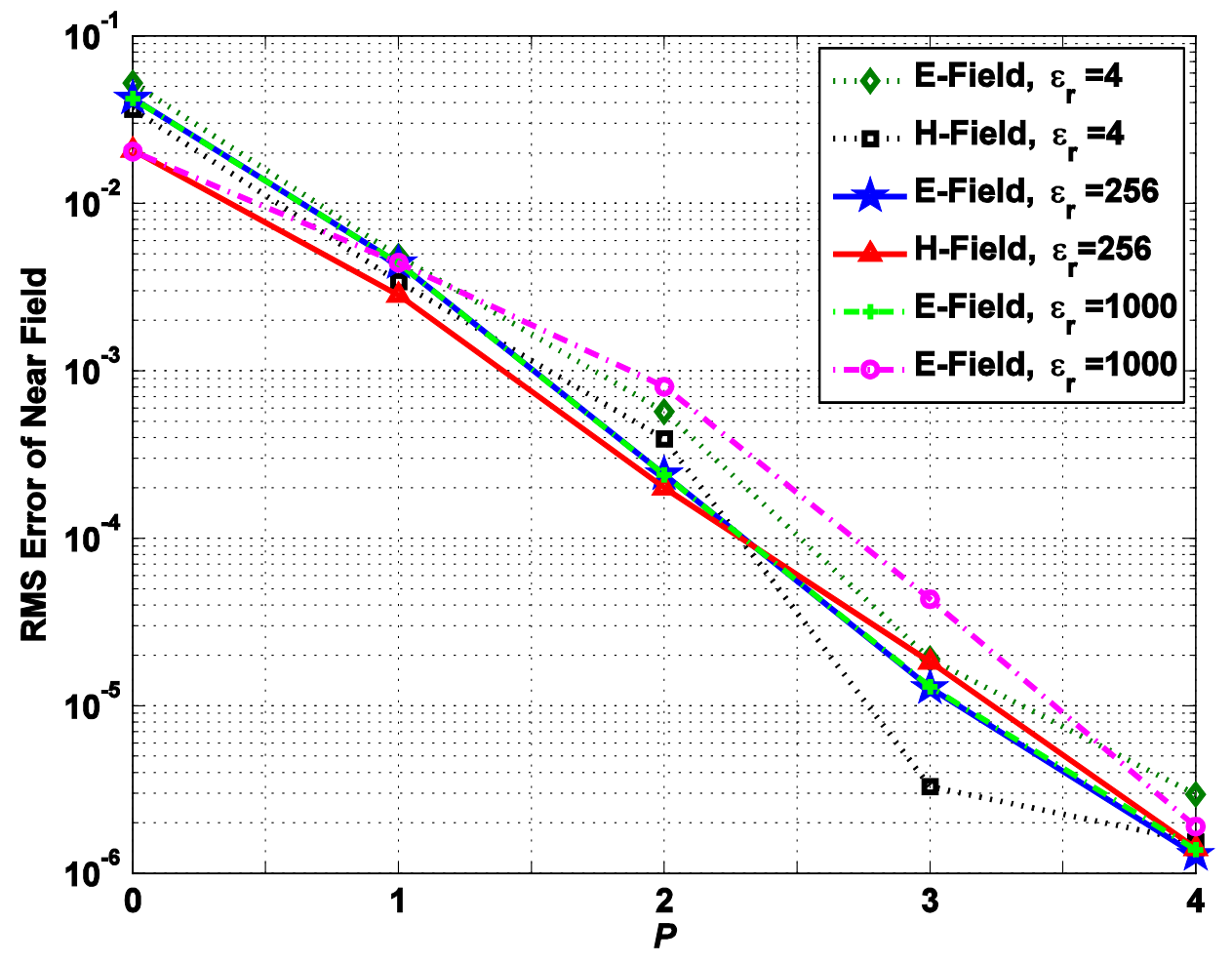

Figure 7: The high order (HO) convergence of the error in near electric and magnetic fields at $1 \mathrm{~Hz}$ computed by the AVIE formulation applied to the $1 \mathrm{~m}$ radius dielectric sphere for different values of $\varepsilon_{r}$ 


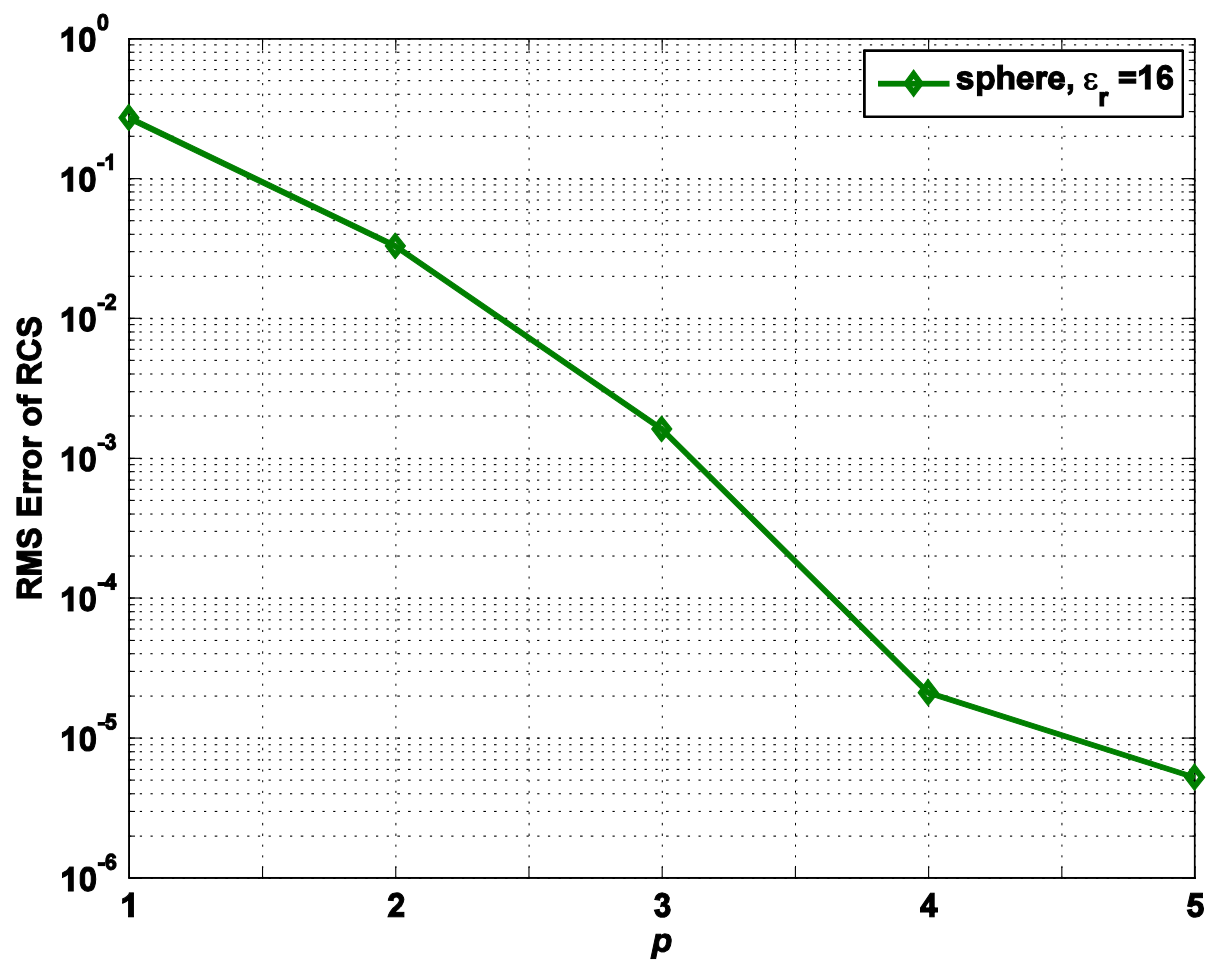

Figure 8: The high order (HO) convergence of the error in radar cross section (RCS) computed by the AVIE for the $1 \mathrm{~m}$ radius dielectric sphere with $\varepsilon_{r}=16$ at $50 \mathrm{MHz}$. 


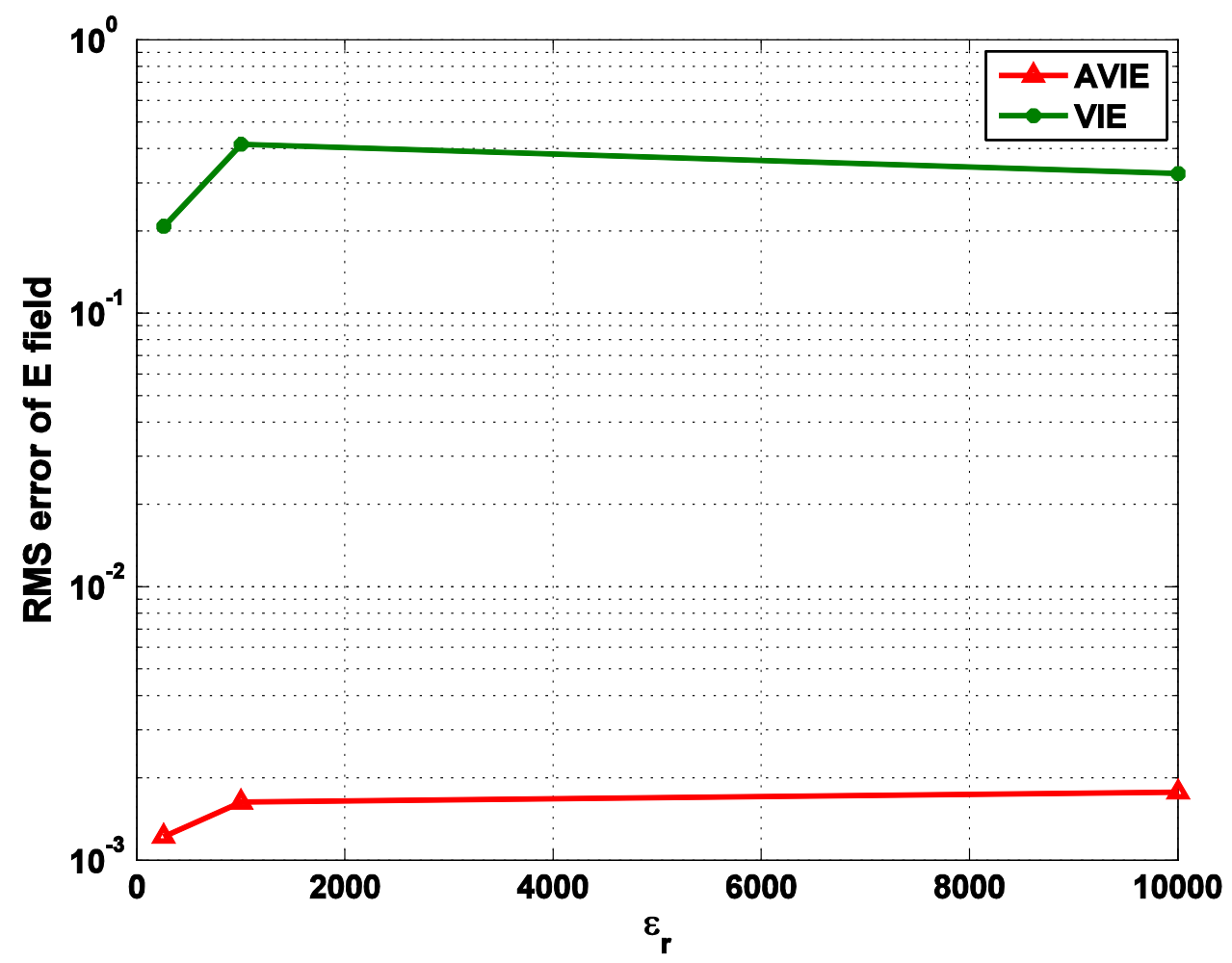

Figure 9: the error of near electric fields at $1 \mathrm{~Hz}$ versus relative permittivity for a $1 \mathrm{~m}$ radius dielectric sphere computed by AVIE and VIE formulations with LOGOS. 


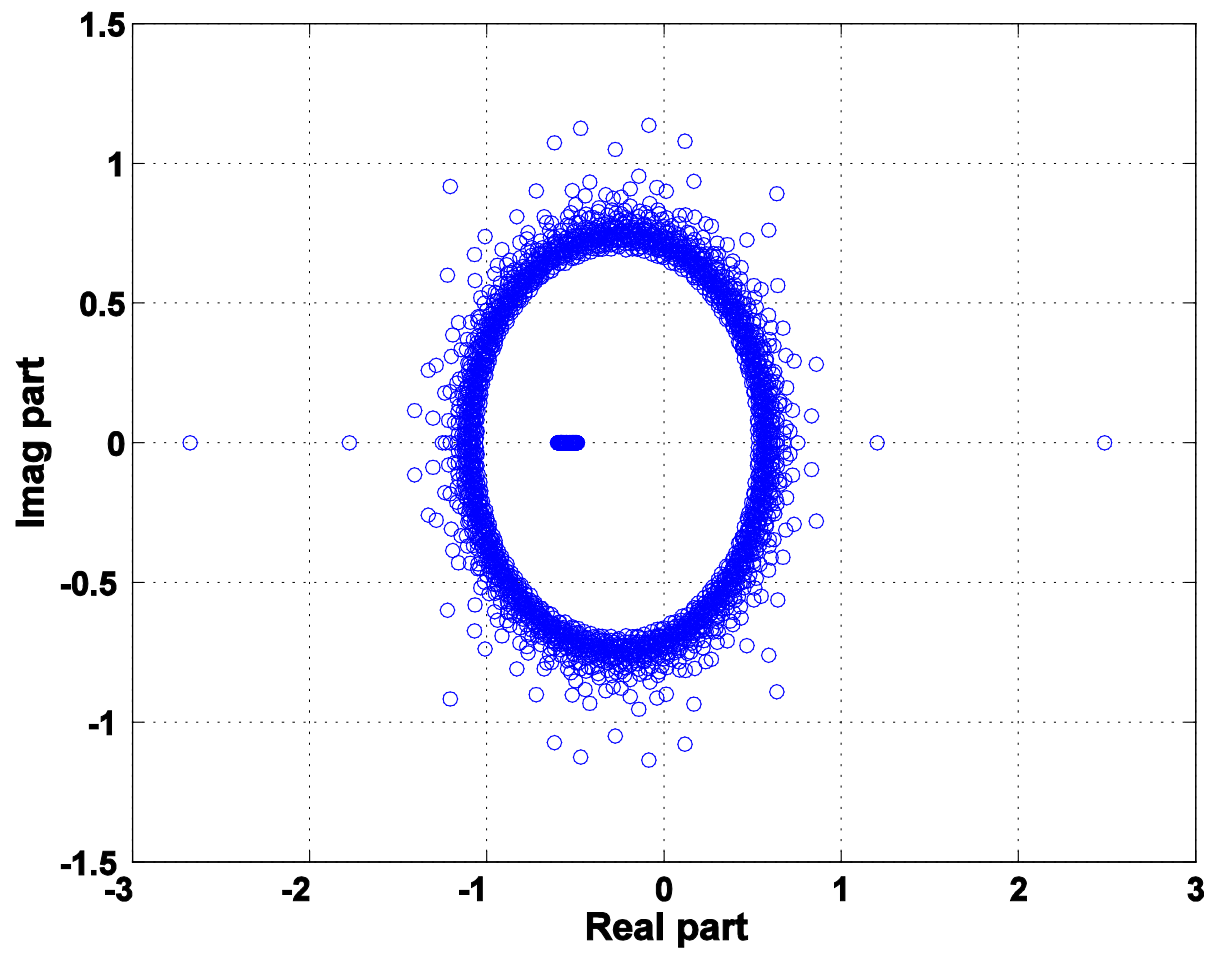

Figure 10: The eigenvalues of the preconditioned AVIE system matrix using simultaneous left and right diagonal scaling algorithm for scattering from the dielectric sphere with $\varepsilon_{r}=400$ at $1 \mathrm{~Hz}$ 


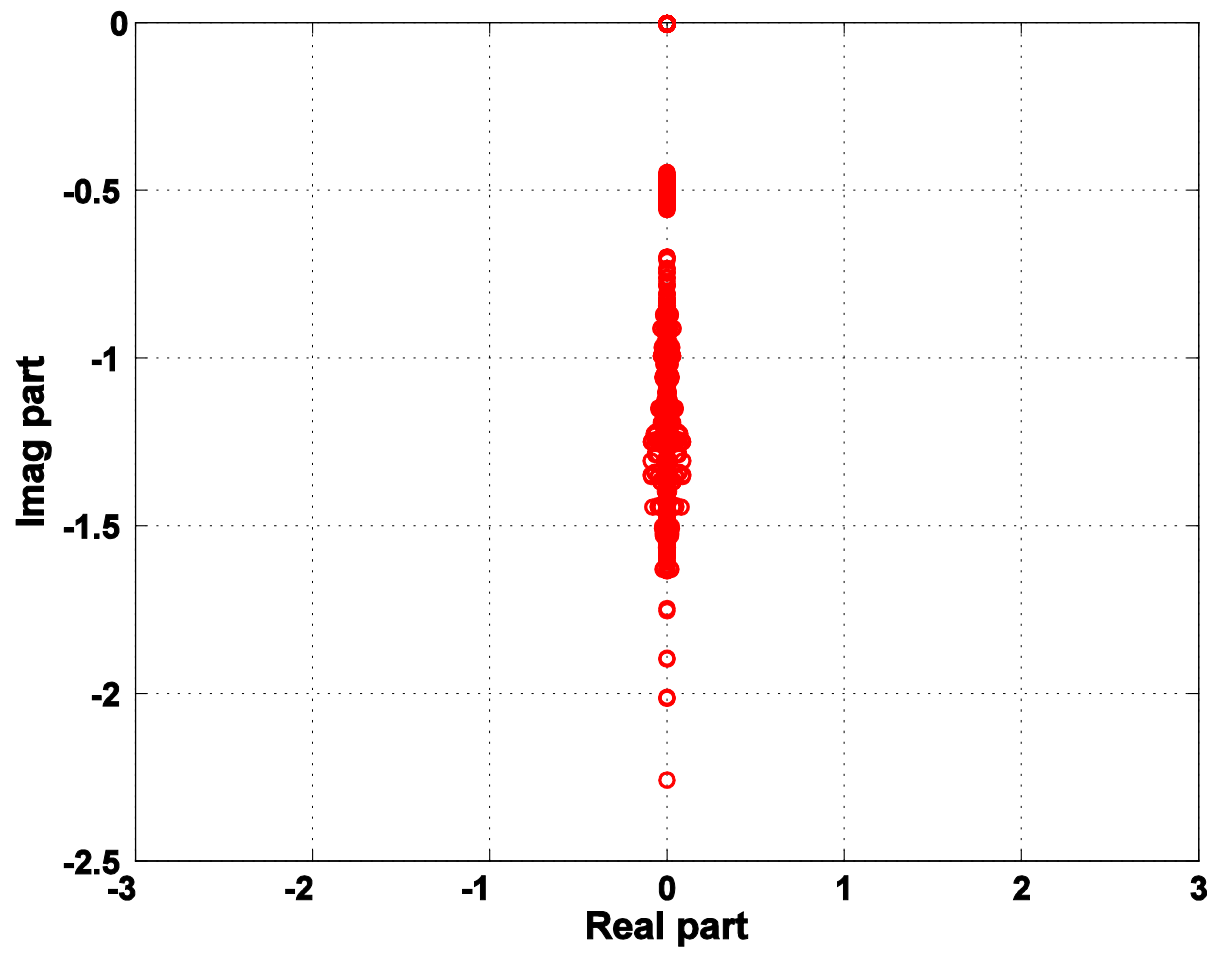

Figure 11: The eigenvalues of the preconditioned VIE system matrix using simultaneous left and right diagonal scaling algorithm for scattering from the dielectric sphere with $\varepsilon_{r}=400$ at $1 \mathrm{~Hz}$ 


\section{Chapter 5. THE CONSTRAINED LCN DisCRETIZATION METHOD}

As it was discussed in the previous chapters, the HO LCN method provides a point based strategy to solve integral equations. While the HO LCN provides many advantages of point based schemes, such as computational efficiency and simplicity of implementation, the underlying currents and field vector quantities are not continuous across the boundaries separating cells of the mesh. This potential shortcoming explains the somewhat less accurate results of the low-order LCN compared to the div conforming MOM [26]. Recently, a linear transformation of the first order LCN method to the RWG MOM for triangular meshes has been reported [30]. The aforementioned transformation improves the accuracy of the first order LCN solutions and reduces the number of unknowns. However, the generalization of the method to high orders and arbitrary mesh element types is not straightforward. A constrained locally corrected Nyström (CLCN) method is developed in this chapter. The CLCN method enables the imposition of normal continuity on underlying vector quantities across mesh element boundaries. This is accomplished by deriving appropriate transformation vectors through simple algebraic analyses of local, homogeneous constraint conditions. Compared to the LCN method, the CLCN method improves the condition numbers of the system matrix, it reduces computational costs through a reduction in the number of degrees of freedom (DOFs), and it improves accuracy for sharp geometries. In the case of the magnetic field integral equation (MFIE), it is shown that the CLCN method yields stable condition numbers as the order increases [28, 49]. 


\subsection{Connection of LCN to the Method of Moment}

The LCN method can be related to the method of moment by expressing the current and test vectors in terms of appropriate basis functions [23]. In the following we assume that we are involved with surface integral equations. Referring to (3.88), the current on the $n$th source quadrilateral cell is expanded in terms of $2 N_{q}$ mixed order basis functions $\mathbf{F}_{n}^{k, j}(\mathbf{r})$. Applying (3.39) we obtain [28]:

$$
\mathrm{J}^{i}(\mathbf{r})=\mathbf{a}^{i}(\mathbf{r}) \cdot \mathbf{J}(\mathbf{r})
$$

From (3.88) and (5.1) :

$$
\bar{J}_{n}=\left[L_{n}\right]^{T} \cdot \bar{\beta}_{n}
$$

holds between current coefficients and the mixed-order basis function coefficients where $\bar{J}_{n}$ was is defined in (3.56); $\left[L_{n}\right]$ is the $2 N_{q} \times 2 N_{q}$ block diagonal matrix defined in (3.46). In (5.2), the vector $\bar{\beta}_{n}$ is defined as:

$$
\bar{\beta}_{n}=\left[\begin{array}{l}
\bar{\beta}_{n}^{1} \\
\bar{\beta}_{n}^{2}
\end{array}\right]
$$

where, the length $N_{q}$ vector $\bar{\beta}_{n}^{j}, j=1,2$, has $k$ th entry:

$$
\left(\bar{\beta}_{n}^{j}\right)_{k}=\beta_{n}^{k, j}
$$

where, $\beta_{n}^{k, j}$ are the coefficients of the basis functions in the current expansion defined in (3.88). With (5.2), the point-matched MOM equivalent to the LCN discretization in (3.54) becomes [23]:

$$
\left(\left[Z^{\mathrm{LCN}}\right]\left[\mathrm{L}^{\mathrm{s}}\right]^{T}\right) \bar{\beta}=\overline{\mathrm{f}}
$$

Here, $\left[\mathrm{L}^{\mathrm{s}}\right]$ is a $2 N_{q} N_{c} \times 2 N_{q} N_{c}$ block diagonal matrix defined as: 


$$
\left[\mathrm{L}^{\mathrm{s}}\right]=\left[\begin{array}{cccc}
{\left[L_{1}\right]} & {[0]} & \cdots & {[0]} \\
{[0]} & {\left[L_{2}\right]} & \cdots & \vdots \\
\vdots & \vdots & \ddots & {[0]} \\
{[0]} & \cdots & {[0]} & {\left[L_{N_{c}}\right.}
\end{array}\right],
$$

where, $N_{c}$ is the number of cells in the mesh, and the length $2 N_{q} N_{c}$ vector $\bar{\beta}$ comprises all $N_{c}$ vectors $\bar{\beta}_{n}$ from (5.3):

$$
\bar{\beta}=\left[\begin{array}{c}
\bar{\beta}_{1} \\
\vdots \\
\bar{\beta}_{n} \\
\vdots \\
\bar{\beta}_{N_{c}}
\end{array}\right] .
$$

Equation (5.5) is a set of point-wise constraints on the basis coefficients, $\beta_{n}^{k, j}$. In order to similarly recast the LCN field side to a MOM form, we introduce $N_{t}$ test functions $\mathbf{T}_{m}^{k}(\mathbf{r})$ with support over the $m$ th observation cell. $\mathbf{T}_{m}^{k}(\mathbf{r})$ is decomposed in unitary vector directions:

$$
\mathbf{T}_{m}^{k}(\mathbf{r})=T_{m}^{k, 1}(\mathbf{r}) \mathbf{a}_{1}(\mathbf{r})+T_{m}^{k, 2}(\mathbf{r}) \mathbf{a}_{2}(\mathbf{r})
$$

where, $T_{m}^{k, i}(\mathbf{r})=\mathbf{a}^{i}(\mathbf{r}) \cdot \mathbf{T}_{m}^{k}(\mathbf{r})$. Consequently, on the right hand side of (5.5), the MOM testing is performed by computing the inner product of the test functions $\mathbf{T}_{m}^{k}(\mathbf{r})$ and the incident field over the $m$ th field cell. Using (5.8) and approximating the inner product of a test function and the incident field by the specified mixed-order quadrature rules on the $m$ th field cell, one obtains:

$$
\int_{S_{m}} \mathbf{T}_{m}^{k}(\mathbf{r}) \cdot \mathbf{f}^{i n c}(\mathbf{r}) d s \approx \sum_{i=1}^{2} \sum_{q=1}^{N_{q}} \omega_{q_{m}}^{i} T_{m}^{k, i}(\mathbf{r}) \mathbf{a}_{i}\left(\mathbf{r}_{q_{m}}^{i}\right) \cdot \mathbf{f}^{i n c}\left(\mathbf{r}_{q_{m}}^{i}\right)
$$


In matrix notation, (5.9) can be expressed as:

$$
\bar{\alpha}_{m}=\left[L_{m}^{\mathrm{t}}\right] \cdot \bar{f}_{m}
$$

where $\bar{f}_{m}$ is the LCN right hand side defined in (3.56). The $N_{t} \times 2 N_{q}$ matrix $\left[L_{m}^{\mathrm{t}}\right]$ has $\left(k, q^{i}\right)$ th entry:

$$
\left[L_{m}^{\mathrm{t}}\right]_{k, q^{i}}=\mathbf{a}^{i}\left(\mathbf{r}_{q_{m}}^{i}\right) \cdot \mathbf{T}_{m}^{k}\left(\mathbf{r}_{q_{m}^{i}}^{i}\right)
$$

The length $N_{t}$ vector $\bar{\alpha}_{m}$ has $k$ th entry defined as:

$$
\left(\bar{\alpha}_{m}\right)_{k}=-\int_{S_{m}} \mathbf{T}_{m}^{k}(\mathbf{r}) \cdot \mathbf{f}^{i n c}(\mathbf{r}) d s
$$

Application of (5.10) to all $N_{c}$ cells provides the following relationship:

$$
\bar{\alpha}=\left[\mathrm{L}^{\mathrm{t}}\right] \cdot \bar{f},
$$

between the right hand sides of the MOM and the LCN method, where the length $N_{t} N_{c}$ vector $\bar{\alpha}$ incorporates all $N_{c}$ vectors $\bar{\alpha}_{m}$ represented in (5.10):

$$
\bar{\alpha}=\left[\begin{array}{c}
\bar{\alpha}_{1} \\
\vdots \\
\bar{\alpha}_{m} \\
\vdots \\
\bar{\alpha}_{N_{c}}
\end{array}\right] .
$$

Combining (5.5) and (5.13) gives:

$$
\left(\left[\mathrm{L}^{\mathrm{t}}\right]\left[\mathrm{Z}^{\mathrm{LCN}}\right]\left[\mathrm{L}^{\mathrm{s}}\right]^{T}\right) \bar{\beta}=\bar{\alpha}
$$

Equation (5.15) constrains the vector of unknown current basis coefficients, $\bar{\beta}$, via a set of reactions with test functions . Thus, we define the MOM-via-LCN impedance matrix as the product of terms on the left side of (5.15): 


$$
\left[\mathrm{Z}^{\mathrm{MLCN}}\right]=\left[\mathrm{L}^{\mathrm{t}}\right]\left[\mathrm{Z}^{\mathrm{LCN}}\right]\left[\mathrm{L}^{\mathrm{s}}\right]^{T}
$$

Based on (5.16), the local MOM-via-LCN impedance matrix $\left[z_{m, n}^{\mathrm{MLCN}}\right]$ for the interaction of the $n$th source cell and the $m$ th observation cell is then:

$$
\left[z_{m, n}^{\mathrm{MLCN}}\right]=\left[L_{m}^{\mathrm{t}}\right]\left[z_{m, n}^{\mathrm{LCN}}\right]\left[L_{n}\right]^{T}
$$

For a standard LCN discretization on a quadrilateral mesh, the basis and test functions underlying this MOM-via-LCN system are Legendre polynomials. In general, (5.16) will not be strictly symmetric even if the source and field basis sets are the same because the fixed-point quadrature rules used in the LCN discretization only approximate the MOM field test integrals.

\subsection{Constrained LCN}

Divergence-conforming basis functions are widely used to develop MOM discretizations of surface integral equations. A critical feature of such basis functions is that the divergence of the current is finite at all points. It is straightforward to obtain a similar result from an LCN discretization by imposing the following constraint on all the edges of the surface mesh [36]:

$$
\mathbf{e}_{n 1}(\mathbf{r}) \cdot \mathbf{J}_{n 1}(\mathbf{r})+\mathbf{e}_{n 2}(\mathbf{r}) \cdot \mathbf{J}_{n 2}(\mathbf{r})=0
$$

where $\mathbf{e}_{n 1}$ and $\mathbf{e}_{n 2}$ are used to indicate the outward vectors emanating from the cells $n_{1}$ and $n_{2}$ that are normal to the edge shared by the two cells at the point indicated by the vector $\mathbf{r}$. Similarly, $\mathbf{J}_{n 1}$ and $\mathbf{J}_{n 2}$ indicate the current on opposite sides of the edge at the point $\mathbf{r}$. At open edges (5.18) reduces to:

$$
\mathbf{e}_{n}(\mathbf{r}) \cdot \mathbf{J}_{n}(\mathbf{r})=0
$$


where the open edge belongs to cell $n$.

Equation (5.18) states that the current leaving a cell at a given point on its boundary must equal the current entering a neighboring cell that shares the same boundary point. This constraint was previously used in developing a global Helmholtz decomposition in [17]. In the following, the constraint is locally applied to LCN basis and test functions,

yielding a constrained LCN (CLCN) method. Because the constraint (24) is simply based on samples of vector quantities at cell boundaries, it is straightforward to apply in different situations, including high-order discretizations.

Equation (5.18) will be imposed in two steps. First, it is used to constrain the LCN bases on individual cells; this provides a transformation of the LCN basis corresponding to currents that do not flow across the cell boundary. Next, (5.18) is imposed on pairs of surface cells that share a common edge. After excluding DOF found in the first step, this yields the subspace of the LCN basis corresponding to continuous current flow across shared boundaries. Any remaining combinations of the LCN DOF not found in these two steps are discarded. This results in a smaller system matrix. Numerical examples demonstrate that solutions obtained using the reduced CLCN system matrix are at least as accurate as those obtained from the larger LCN system.

\subsubsection{Transformation vectors on individual cells}

To develop appropriate local constraint matrices for the currents that do not flow across the edges of an individual cell, (5.19) is enforced on boundary points of the $n$th cell:

$$
\mathbf{e}_{n}\left(\mathbf{r}_{b q_{n}}\right) \cdot \mathbf{J}_{n}\left(\mathbf{r}_{b q_{n}}\right)=0
$$


where $\mathbf{r}_{b q_{n}}, b q=1, \ldots, N_{b q}$, are the edge quadrature points on the boundary of the nth cell. Selecting the mixed-order Legendre polynomial functions as the current expansion functions, $\mathbf{F}_{n}^{k, j}$, in (3.88) and collecting the constraint (5.20) on all boundary points of the cell, we obtain the following matrix equation:

$$
\left[\mathrm{C}_{n}\right] \cdot \bar{\beta}_{n}=\overline{\mathbf{0}}
$$

where $\overline{\mathbf{0}}$ is a zero vector (of appropriate size) and $\bar{\beta}_{n}$ is defined in(5.7). There are $2 N_{q}$ mixed-order basis functions associated with the $n$th cell. Thus, $\left[\mathrm{C}_{n}\right]$ with size $N_{b q} \times 2 N_{q}$ is defined as:

$$
\left[\mathrm{C}_{n}\right]=\left[\left[\mathrm{C}_{n}^{1}\right] \quad\left[\mathrm{C}_{n}^{2}\right]\right]
$$

where, $\left[\mathrm{C}_{n}^{j}\right], j=1,2$, is a $N_{b q} \times N_{q}$ matrix with $(b q, k)$ th entry:

$$
\left[\mathrm{C}_{n}^{j}\right]_{b q, k}=\mathbf{e}_{n}\left(\mathbf{r}_{b q_{n}}\right) \cdot \mathbf{F}_{n}^{k, j}\left(\mathbf{r}_{b q_{n}}\right)
$$

It should be mentioned that in developing the local constraints, the mixed order Legendre polynomial bases are normalized to improve the stability of the resulting formulation. Thus, considering (3.91) and (3.92) the normalized mixed order Legendre basies $\mathbf{F}_{n}^{k, j}$ in (5.23) are represented as:

$$
\begin{gathered}
\mathbf{F}_{n}^{k, 1}=\frac{\sqrt{(2 m+1)(2 l+1)} P_{m}\left(u^{1}\right) P_{l}\left(u^{2}\right)}{\sqrt{g_{s}}} \mathbf{a}_{1}, \quad(m=0, \ldots, p+1 ; l=0, \ldots, p) \\
\mathbf{F}_{n}^{k, 2}=\frac{\sqrt{(2 m+1)(2 l+1)} P_{m}\left(u^{1}\right) P_{l}\left(u^{2}\right)}{\sqrt{g_{s}}} \mathbf{a}_{2}, \quad(m=0, \ldots, p ; l=0, \ldots, p+1)
\end{gathered}
$$

The non-trivial solutions of (5.21) correspond to currents that do not flow across the edges of the $n$th cell and comprise the null space of $\left[C_{n}\right][13]$. 
For a $p$ th order LCN discretization with a mixed-order representation of the currents on quadrilateral cells, the currents flowing tangential to a specific edge are order $p+1$ along the edge, and the currents flowing normal to the edge are order $p$ along the edge [24]. As a result, in order to fully constrain the order $p$ current normal to the edge in (5.18) and (5.19), at least $p+1$ quadrature points are needed along the edge. (While more than $p^{+1}$ points can be used, the additional constraints will be linearly dependent; such an approach is not considered here.) Thus, $p+1$ quadrature points are allocated to each edge, and the number of quadrature points on the four edges forming the boundary of the quadrilateral cell is $N_{b q}=4(p+1)$. For a zero-order LCN discretization, $\left[\mathrm{C}_{n}\right]$ is a $4 \times 4$ full rank matrix; thus, there is no non-trivial solution associated with (5.21). However, for $p \geq 1$, the matrix $\left[\mathrm{C}_{n}\right]$ has a number of rows, $N_{b q}$, less than the number of columns, $2 N_{q}$. Since there is no linear dependence between rows of the constraint matrix, the rank of $\left[\mathrm{C}_{n}\right]$ is equal to the number of rows $N_{b q}$. Therefore, the nullity of $\left[\mathrm{C}_{n}\right], n u l_{C}$, is [13]:

$$
n u l_{\mathrm{C}}=2 N_{q}-N_{b q}=2 p(p+1)
$$

The $n u l_{C}$ orthonormal basis vectors spanning the null space of $\left[\mathrm{C}_{n}\right]$ are the non-trivial solutions of (5.21). These bases are obtained using an SVD:

$$
\left[\mathrm{C}_{n}\right]=\left[\mathbf{U}_{n}\right]\left[\mathbf{S}_{n}\right]\left[\mathbf{V}_{n}\right]^{H},
$$

where $\left[\mathbf{U}_{n}\right]$ and $\left[\mathbf{V}_{n}\right]$ are $N_{b q} \times N_{b q}$ and $2 N_{q} \times 2 N_{q}$ unitary matrices, respectively, that comprise the left and right singular vectors of $\left[\mathrm{C}_{n}\right]$. The diagonal matrix $\left[\mathbf{S}_{n}\right]$ contains the singular values (assumed to be decreasingly ordered) of $\left[\mathrm{C}_{n}\right]$. The last $n u l_{C}$ columns of $\left[\mathbf{V}_{n}\right]$ form the orthonormal basis for the null space of $\left[\mathrm{C}_{n}\right]$ in (5.21). Thus, define 
the $2 N_{q} \times n u l_{C}$ matrix $\left[\mathbf{v}_{\mathrm{C}_{n}}\right]$ to be the last $n u l_{C}$ columns of $\left[\mathbf{V}_{n}\right]$ which correspond to the zero singular vectors. The matrix $\left[\mathbf{v}_{\mathrm{C}_{n}}\right]$ includes the linearly independent column vectors that are non-trivial solutions of (5.21). Applying (5.23) and substituting the column vectors of $\left[\mathbf{v}_{\mathrm{C}_{n}}\right]$ in place of $\bar{\beta}_{n}$ we obtain:

$$
\mathbf{e}_{n}\left(\mathbf{r}_{b q_{n}}\right) \cdot \mathbf{f}_{\mathrm{C}_{n}}^{t}\left(\mathbf{r}_{b q_{n}}\right)=0
$$

where,

$$
\mathbf{f}_{\mathrm{C}_{n}}^{t}(\mathbf{r})=\sum_{j=1}^{2} \sum_{k=1}^{N_{q}}\left[\mathbf{v}_{\mathrm{C}_{n}}\right]_{k^{\prime}, t} \mathbf{F}_{n}^{k, j}(\mathbf{r})
$$

In (5.29) $t=1, \ldots, N_{\mathbf{v}_{\mathbf{C}}}$ where, $N_{\mathbf{v}_{\mathbf{C}}}=n u l_{C} \cdot\left[\mathbf{v}_{\mathrm{C}_{n}}\right]_{k^{\prime}, t}$, is the $\left(k^{\prime}, t\right)$ th entry of $\left[\mathbf{v}_{\mathrm{C}_{n}}\right]$ where, $k^{\prime}=(j-1) N_{q}+k$. The functions $\mathbf{f}_{\mathrm{C}_{n}}^{t}(\mathbf{r})$ in (5.28) are the set of $N_{\mathbf{v}_{\mathrm{c}}}$ orthonormal functions constructed from the mixed-order LCN basis functions $\mathbf{F}_{n}^{k, j}(\mathbf{r})$ to have a zero normal component at all edges of the $n$th quadrilateral cell.

\subsubsection{Transformation vectors associated with edges}

In addition to the basis functions associated with individual cells, it is also necessary to construct bases that provide continuous flow across the edges shared by neighboring cells. These edge functions are constructed by imposing normal current continuity on two adjacent cells, $n_{1}$ and $n_{2}$, which share an interior edge. The constraint (5.18) is used at quadrature points on the interior edge shared by the two cells, and the constraint (5.19) is imposed at quadrature points on the non-shared edges of the two neighboring cells. Collecting the constraints and expanding the currents in terms of the normalized mixedorder LCN basis functions $\mathbf{F}_{n}^{k, j}$ yields: 


$$
\left[\mathrm{E}_{n_{1}, n_{2}}\right]\left[\begin{array}{l}
\bar{\beta}_{n_{1}} \\
\bar{\beta}_{n_{2}}
\end{array}\right]=\overline{\mathbf{0}}
$$

where $\bar{\beta}_{n}$ was defined in (5.3), and

$$
\left[\mathrm{E}_{n_{1}, n_{2}}\right]=\left[\left[\mathrm{E}_{n_{1}}\right]\left[\mathrm{E}_{n_{2}}\right]\right] \text {. }
$$

The $\left[\mathrm{E}_{n}\right]$ has size $N_{b q_{\eta_{1}, n_{2}}} \times 2 N_{q}$, where, $N_{b q_{\eta_{1}, n_{2}}}$ is the number of the edge quadrature points on the shared edge between neighboring cells $n_{1}$ and $n_{2}$. The matrix $\left[\mathrm{E}_{n}\right]$ is defined as:

$$
\left[\mathrm{E}_{n}\right]=\left[\left[\mathrm{E}_{n}^{1}\right]\left[\mathrm{E}_{n}^{2}\right]\right]
$$

where, the elements of $\left[\mathrm{E}_{n}^{j}\right]$ are defined in a manner similar to (5.23):

$$
\left[\mathrm{E}_{n}^{j}\right]_{b q, k}=\mathbf{e}_{n}\left(\mathbf{r}_{b q_{n_{1}, n_{2}}}\right) \cdot \mathbf{F}_{n}^{k, j}\left(\mathbf{r}_{b q_{n_{1}, n_{2}}}\right)
$$

where, $b q=1, \ldots, N_{b q_{q_{1}, n_{2}}}$. It should be noted that if $\mathbf{r}_{b q_{n_{1}, n_{2}}}$ is not located on the boundary of the $n$th cell $\left[\mathrm{E}_{n}^{j}\right]_{b q, k}=0$.

The desired edge transformation vectors $\left[\mathbf{v}_{\mathrm{E}_{n_{1}, n_{2}}}\right]$ are the non-trivial solutions of (5.30) which are the right singular vectors associated with the zero singular values of the SVD of $\left[\mathrm{E}_{n_{1}, n_{2}}\right]$ :

$$
\left[\mathrm{E}_{n_{1}, n_{2}}\right]=\left[\mathbf{U}_{\mathrm{E}}\right]\left[\mathbf{S}_{\mathrm{E}}\right]\left[\mathbf{V}_{\mathrm{E}}\right]^{H}
$$

As explained in the previous section, for a $p$ th-order LCN discretization, $p+1$ quadrature points are assigned to an individual edge. Therefore, the number of quadrature points on the seven edges associated with the two neighboring quadrilateral cells is 
$N_{b q_{n_{1}, n_{2}}}=7(p+1)$. Thus, for all orders, $\left[\mathrm{E}_{n_{1}, n_{2}}\right]$ has fewer rows than columns. The rows of $\left[E_{n_{1}, n_{2}}\right]$ are linearly independent, so its rank is equal to the number of rows; and the nullity $\operatorname{nul}_{\mathrm{E}_{n_{1}, n_{2}}}$ of $\left[\mathrm{E}_{n_{1}, n_{2}}\right]$ is given by:

$$
\operatorname{nul}_{\mathrm{E}_{n_{1}, n_{2}}}=4 N_{q}-N_{b q_{n_{1}, n_{2}}}=4 p^{2}+5 p+1
$$

Hence, the number of columns of $\left[\mathbf{v}_{\mathrm{E}_{n_{1}, n_{2}}}\right]$ is also $n u l_{\mathrm{E}_{n_{1}, n_{2}}}$.

The null space of $\left[E_{n_{1}, n_{2}}\right]$ includes vectors that satisfy (5.19) individually on cells $n_{1}$ and $n_{2}$. These transformation vectors are already provided by $\left[\mathbf{v}_{\mathrm{C}_{n}}\right]$ and should be removed from the edge basis. To this end, define:

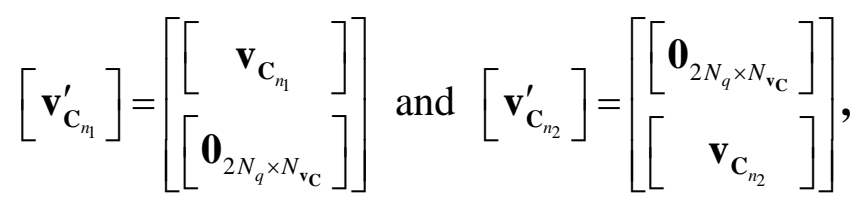

where $\left[\mathbf{v}_{\mathrm{C}_{n}}\right]$ includes the transformation vectors associated with $n$th cell. The column vectors of $\left[\mathbf{v}_{\mathrm{C}_{n}}^{\prime}\right]$ are non-trivial solutions of (5.30) corresponding to currents that do not flow across the edges of the two adjacent cells. Since $\left[\mathbf{v}_{\mathrm{C}_{n_{1}}}^{\prime}\right]$ and $\left[\mathbf{v}_{\mathrm{C}_{n_{2}}}^{\prime}\right]$ are orthonormal, it follows that they are an orthonormal subspace of the null space of $\left[E_{n_{1}, n_{2}}\right]$, which makes it straightforward to remove them. As a result, the number $N_{\mathbf{v}_{\mathbf{E}}}$ of singular vectors that correspond to the continuous current flow across the shared interior edge is obtained by using (5.26) and (5.35) to be:

$$
N_{\mathbf{v}_{\mathbf{E}}}=n u l_{\mathbf{E}_{n_{1}, n_{2}}}-2 N_{\mathbf{v}_{\mathbf{C}}}=p+1
$$

Consequently, in order to project out the redundant solutions $\left[\mathbf{v}_{\mathrm{C}_{n}}^{\prime}\right]$, define [13]: 


$$
\left[\mathbf{p}_{\mathrm{E}_{n_{1}, n_{2}}}\right]=\left[\mathbf{v}_{\mathrm{E}_{n_{1}, n_{2}}}\right]-\sum_{i=1}^{2}\left[\mathbf{v}_{\mathrm{C}_{n_{i}}}^{\prime}\right]\left[\mathbf{v}_{\mathrm{C}_{n_{i}}}^{\prime}\right]^{H}\left[\mathbf{v}_{\mathrm{E}_{n_{1}, n_{2}}}\right],
$$

where $\left[\mathbf{v}_{\mathrm{E}_{n_{1}, n_{2}}}\right]$ includes the orthonormal basis for the null space of $\left[\mathrm{E}_{n_{1}, n_{2}}\right]$ which are the last $n u l_{\mathrm{E}_{n_{1}, n_{2}}}$ columns of the right unitary matrix obtained through the SVD of $\left[\mathrm{E}_{n_{1}, n_{2}}\right]$. The orthonormal bases for the column space of the projected matrix $\left[\mathbf{p}_{\mathbf{E}_{n_{1}, n_{2}}}\right]$ provide the non-trivial solutions of (5.30) corresponding to the shared edge. Consequently, we define $\left[\mathbf{v}_{\mathrm{E}_{l}}\right]$ to be the first $N_{\mathbf{v}_{\mathbf{E}}}$ columns of $\mathbf{U}_{\mathbf{p}}$ which is obtained through SVD of $\left[\mathbf{p}_{\mathbf{E}_{n_{1}, n_{2}}}\right]$ :

$$
\left[\mathbf{p}_{\mathbf{E}_{1, n_{2}}}\right]=\left[\mathbf{U}_{\mathbf{p}}\right]\left[\mathbf{S}_{\mathbf{p}}\right]\left[\mathbf{V}_{\mathbf{p}}\right]^{H}
$$

Consequently, the matrix $\left[\mathbf{v}_{\mathrm{E}_{l}}\right]$ of size $4 N_{q} \times N_{\mathbf{v}_{\mathbf{E}}}$ includes the linearly independent column vectors that are non-trivial solutions of (5.30). Similar to the discussion in (5.28) through (5.29), we derive a set of CLCN basis functions $\mathbf{f}_{\mathrm{E}_{l}}^{t}$ spanning the two adjacent cells through the transformation:

$$
\mathbf{f}_{\mathrm{E}_{l}}^{t}(\mathbf{r})=\left\{\begin{array}{cc}
\sum_{j=1}^{2} \sum_{k=1}^{N_{q}}\left[\mathbf{v}_{\mathrm{E}_{l}}\right]_{k^{\prime}, t} \mathbf{F}_{n_{1}}^{k, j}(\mathbf{r}) & \mathbf{r} \in S_{n_{1}} \\
\sum_{j=1}^{2} \sum_{k=1}^{N_{q}}\left[\mathbf{v}_{\mathrm{E}_{l}}\right]_{k^{\prime}+2 N_{q}, t} \mathbf{F}_{n_{2}}^{k, j}(\mathbf{r}) & \mathbf{r} \in S_{n_{2}}
\end{array}\right.
$$

where, $k^{\prime}=(j-1) N_{q}+k \cdot\left[\mathbf{v}_{\mathrm{E}_{l}}\right]_{k, t}$ is the $(k, t)$ th entry of $\left[\mathbf{v}_{\mathrm{E}_{l}}\right]$. The $\mathbf{f}_{\mathrm{E}_{l}}^{t}$ in $(5.40)$ provide a set of functions whose normal components are continuous across the lth shared edge and zero at all other edges . 


\subsubsection{CLCN local interactions}

Equation (5.17) provides the MOM-via-LCN (MLCN) sub-matrices that represent interactions between the specified Legndre polynomial test and basis functions on the $m$ th and $n$th cells. In the following, analogous CLCN sub-matrices are developed for reactions between the specific cell and edge functions defined in (5.29) and (5.40), which were derived via constraints (5.21) and (5.30). It is subsequently shown that the resultant CLCN interaction matrices are simply a product of the original MLCN matrix with the local transformation vectors associated with cells and edges defined in previous sections.

\section{A. Cell-to-cell interactions}

Based on (5.17), the local CLCN impedance matrix corresponding to the interaction of the $m$ th observation cell and $n$th source cell using the CLCN cell basis functions in (5.29) as the source basis and testing functions is expressed as:

$$
\left[\mathrm{Z}_{\mathrm{C}_{m}, \mathrm{C}_{n}}^{\mathrm{CLCN}}\right]=\left[L_{\mathrm{C}_{m}}\right]\left[\mathrm{z}_{m, n}^{\mathrm{LCN}}\right]\left[L_{\mathrm{C}_{n}}\right]^{T}
$$

where $\left[L_{C_{n}}\right]$ is a matrix of size $n u l_{C} \times 2 N_{q}$ with $\left(k, q^{i}\right)$ th entry:

$$
\left[L_{\mathrm{C}_{n}}\right]_{k, q^{i}}=\mathbf{a}^{i}\left(\mathbf{r}_{q_{n}}^{i}\right) \cdot \mathbf{f}_{\mathrm{C}_{n}}^{k}\left(\mathbf{r}_{q_{n}}^{i}\right) \text {. }
$$

The matrix $\left[\mathrm{z}_{\mathrm{C}_{m}, \mathrm{C}_{n}}^{\text {CLCN }}\right]$ in (5.41) has size $N_{\mathbf{v}_{\mathrm{c}}} \times N_{\mathbf{v}_{\mathrm{C}}}$. In light of (5.29), the following equation holds:

$$
\left[L_{\mathrm{C}_{n}}\right]^{T}=\left[L_{n}\right]^{T}\left[\mathbf{v}_{\mathrm{C}_{n}}\right]
$$

where $\left[L_{n}\right]$ was defined in (3.46). Combining (5.41) and (5.43), and applying (5.17) we obtain: 


$$
\left[\mathrm{z}_{\mathrm{C}_{m}, \mathrm{C}_{n}}^{\mathrm{CLCN}}\right]=\left[\mathbf{v}_{\mathrm{C}_{m}}\right]^{T}\left[\mathrm{Z}_{m, n}^{\mathrm{MLCN}}\right]\left[\mathbf{v}_{\mathrm{C}_{n}}\right]
$$

Equation (5.44) is a transformation from the local MLCN impedance matrix to the local

CLCN impedance matrix, $\left[\mathrm{z}_{\mathrm{C}_{m}, \mathrm{C}_{n}}^{\mathrm{CLCN}}\right]$, for the $m$ th observation cell and the $n$th source cell. It is observed that the CLCN local matrix is defined in terms of the MLCN local matrix and the right singular vectors associated with nontrivial solutions of the constraint in (5.21).

\section{B. Edge-to-edge interactions}

The local CLCN impedance matrix describing the interaction of the CLCN edge test functions defined in (5.40) associated with the sth observation edge (shared by observation cells $m_{1}$ and $m_{2}$ ) and lth source edge (shared by observation cells $n_{1}$ and $n_{2}$ ) is represented as:

$$
\left[\mathrm{z}_{\mathrm{E}_{s}, \mathrm{E}_{l}}^{\mathrm{CLN}}\right]=\left[L_{\mathrm{E}_{s}}\right]\left[\mathrm{z}_{m_{1,2}, n_{1,2}}^{\mathrm{LCN}}\right]\left[L_{\mathrm{E}_{l}}\right]^{T}
$$

The block matrix $\left[\mathrm{z}_{m_{1,2}, n_{1,2}}^{\mathrm{LCN}}\right]$ in (5.45) contains LCN sub-matrices corresponding to interactions between pairs of observation cells $\left(m_{1}\right.$ and $\left.m_{2}\right)$ and source cells $\left(n_{1}\right.$ and $\left.n_{2}\right)$ :

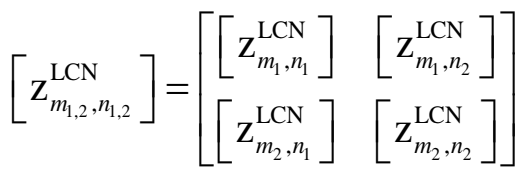

$\left[L_{\mathrm{E}_{l}}\right]$ in (5.45) has size $N_{\mathrm{v}_{\mathrm{E}}} \times 4 N_{q}$. The $\left(k, q_{n_{j}}^{i}\right), i, j \in\{1,2\}$ th entry of $\left[L_{\mathrm{E}_{l}}\right]$ is:

$$
\left[L_{\mathrm{E}_{l}}\right]_{k, q_{n_{j}}}^{i}=\mathbf{a}^{i}\left(\mathbf{r}_{q_{n_{j}}}^{i}\right) \cdot \mathbf{f}_{\mathrm{E}_{l}}^{k}\left(\mathbf{r}_{q_{n_{j}}}^{i}\right)
$$

where, $\mathbf{f}_{\mathrm{E}_{l}}^{k}(\mathbf{r})$ was defined in (5.40). Referring to (5.40), $\left[L_{\mathrm{E}_{l}}\right]$ is represented as:

$$
\left[L_{\mathrm{E}_{l}}\right]^{T}=\left[L_{n_{1}, n_{2}}\right]^{T}\left[\mathbf{v}_{\mathrm{E}_{l}}\right]
$$


where,

$$
\left[L_{n_{1}, n_{2}}\right]=\left[\begin{array}{cc}
{\left[L_{n_{1}}\right]} & {[0]} \\
{[0]} & {\left[L_{n_{2}}\right]}
\end{array}\right],
$$

and where $\left[L_{n}\right]$ was defined in (3.46). Combining (5.41) and (5.48) one obtains:

$$
\left[\mathrm{Z}_{\mathrm{E}_{s}, \mathrm{E}_{l}}^{\mathrm{CLCN}}\right]=\left[\mathbf{v}_{\mathbf{E}_{s}}\right]^{T}\left[\mathbf{z}_{m_{1,2}, n_{1,2}}^{\mathrm{MLCN}}\right]\left[\mathbf{v}_{\mathbf{E}_{l}}\right]
$$

where, $\left[\mathrm{z}_{m_{1,2}, n_{1,2}}^{\mathrm{MLCN}}\right]$ is defined in a way similar to (5.46). The matrix $\left[\mathrm{z}_{\mathrm{E}_{s}, \mathrm{E}_{l}}^{\mathrm{CLCN}}\right]$ has size $N_{\mathbf{v}_{\mathrm{E}}} \times N_{\mathbf{v}_{\mathrm{E}}}$ and provides the local CLCN impedance matrix corresponding to the interaction of the sth observation edge and lth source edge. As observed above, the local CLCN matrix is a projection of the local MLCN matrix onto the right singular vectors obtained as nontrivial solutions to (5.30), which are also orthogonal to the nontrivial solutions of (5.21).

\section{Edge-to-cell and cell-to-edge interactions}

Based on (5.44) and (5.50), the local CLCN impedance matrix describing the interaction of the $m$ th observation cell and the lth source edge (shared by shared by the cells $n_{1}$ and $n_{2}$ ) is represented as:

$$
\left[\mathrm{z}_{\mathrm{C}_{m}, \mathrm{E}_{l}}^{\mathrm{CLCN}}\right]=\left[\mathbf{v}_{\mathrm{C}_{m}}\right]^{T}\left[\left[\mathrm{z}_{m, n_{1}}^{\mathrm{MLCN}}\right]\left[\mathrm{z}_{m, n_{2}}^{\mathrm{MLCN}}\right]\right]\left[\mathbf{v}_{\mathrm{E}_{l}}\right]
$$

The matrix $\left[\mathrm{z}_{\mathrm{C}_{m}, \mathrm{E}_{l}}^{\mathrm{CLCN}}\right]$ has size $N_{\mathbf{v}_{\mathbf{C}}} \times N_{\mathbf{v}_{\mathbf{E}}}$. Furthermore, the local CLCN impedance matrix describing the interaction of the sth observation edge (shared by source cells $m_{1}$ and $m_{2}$ ) and the $n$th source cell is expressed as: 


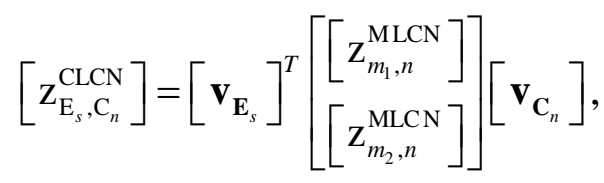

where $\left[\mathrm{z}_{\mathrm{E}_{s}, \mathrm{C}_{n}}^{\text {CLCN }}\right]$ has size $N_{\mathbf{v}_{\mathbf{E}}} \times N_{\mathbf{v}_{\mathbf{C}}}$.

\subsubsection{CLCN global system of equations}

A global representation of the transformed LCN system matrix $\left[\mathrm{Z}^{\mathrm{LCN}}\right]$ in (3.54) can be assembled using the local transformation matrices developed in the previous section. Specifically, the constrained LCN system matrix can be expressed as:

$$
\left[\mathrm{Z}^{\mathrm{CLCN}}\right]=[v]^{T}\left[\mathrm{Z}^{\mathrm{MLCN}}\right][v]
$$

where $\left[\mathrm{Z}^{\mathrm{CLCN}}\right]$ is the CLCN system matrix with a size of $N_{T} \times N_{T}$, and

$$
N_{T}=N_{c} N_{\mathbf{v}_{\mathbf{C}}}+N_{e} N_{\mathbf{v}_{\mathbf{E}}}
$$

$N_{c}$ and $N_{e}$ are respectively the number of quadrilateral cells and interior edges in the mesh. $N_{\mathbf{v}_{\mathrm{C}}}$ and $N_{\mathbf{v}_{\mathrm{E}}}$ were defined respectively in previous sections. $[v]$ is the sparse transformation matrix comprising all the local transformation matrices which can be expressed as,

$$
[v]=\left[\left[v_{\mathrm{C}}\right]\left[v_{\mathrm{E}}\right]\right]
$$

where $\left[v_{\mathrm{C}}\right]$ is a block diagonal matrix of size $2 N_{q} N_{c} \times N_{c} N_{\mathbf{v}_{\mathrm{C}}}$ defined as,

$$
\left[v_{\mathrm{C}}\right]=\operatorname{diag}\left(\left[\mathbf{v}_{\mathrm{C}_{n}}\right]\right), n=1, \ldots, N_{c} \text {. }
$$

$\left[\mathbf{v}_{\mathrm{C}_{n}}\right]$ in (5.56) is the transformation matrix related to the $n$th cell, and the matrix $\left[v_{\mathrm{C}}\right]$ is orthonormal. 
The matrix $\left[v_{\mathrm{E}}\right]$ is a sparse block matrix with size $2 N_{q} N_{c} \times N_{e} N_{\mathrm{v}_{\mathrm{E}}}$ containing the local edge transformation matrices. There are $N_{c}$ row partitions corresponding to the cells of the mesh and $N_{e}$ column block partitions corresponding to the interior edges,

$$
\left[v_{\mathrm{E}}\right]=\left[\begin{array}{ccccccc}
{[0]} & \cdots & {[0]} & \cdots & \cdots & \cdots & {[0]} \\
\vdots & \vdots & \vdots & \vdots & \vdots & \vdots & \vdots \\
{[0]} & \cdots & {\left[\mathbf{v}_{\mathbf{E}_{l}}^{n_{1}}\right]} & \cdots & \cdots & \cdots & {\left[\mathbf{v}_{\mathbf{E}_{N_{e}}}^{n_{1}}\right.} \\
\vdots & \vdots & \vdots & \vdots & \vdots & \vdots & \vdots \\
{\left[\mathbf{v}_{\mathbf{E}_{1}}^{k_{1}}\right]} & \cdots & {[0]} & \cdots & \cdots & \cdots & {[0]} \\
\vdots & \vdots & \vdots & \vdots & \vdots & \vdots & \vdots \\
{[0]} & \cdots & {\left[\mathbf{v}_{\mathbf{E}_{l}}^{n_{2}}\right]} & \cdots & \cdots & \cdots & {[0]} \\
\vdots & \vdots & \vdots & \vdots & \vdots & \vdots & \vdots \\
{\left[\mathbf{v}_{\mathbf{E}_{1}}^{k_{2}}\right]} & \cdots & {[0]} & \cdots & \cdots & \cdots & {\left[\mathbf{v}_{\mathbf{E}_{N_{e}}}^{k_{2}}\right.} \\
\vdots & \vdots & \vdots & \vdots & \vdots & \vdots & \vdots \\
{[0]} & \cdots & {[0]} & \cdots & \cdots & \cdots & {[0]}
\end{array}\right] .
$$

In (5.57), $\left[\mathbf{v}_{\mathbf{E}_{l}}^{n_{1}}\right]$ and $\left[\mathbf{v}_{\mathbf{E}_{l}}^{n_{2}}\right]$ have size $2 N_{q} \times N_{\mathbf{v}_{\mathbf{E}}}$ and are respectively the upper half and lower half of $\left[\mathbf{v}_{\mathbf{E}_{l}}\right]$ defined in previous sections Each column block partition of $\left[v_{\mathrm{E}}\right]$ includes exactly two non-zero blocks of size $2 N_{q} \times N_{\mathbf{V}_{\mathbf{E}}}$. Utilizing (5.16), (5.53) can be represented as:

$$
\left[\mathrm{Z}^{\mathrm{CLCN}}\right]=[v]^{T}\left[\mathrm{~L}^{\mathrm{s}}\right]\left[\mathrm{Z}^{\mathrm{LCN}}\right]\left[\mathrm{L}^{\mathrm{s}}\right]^{T}[v]
$$

where, $\left[\mathrm{L}^{\mathrm{s}}\right]$ is a block diagonal matrix defined in (5.6). Equation (5.58) indicates that the CLCN impedance matrix is obtained via applying sparse left and right transformation matrices to the LCN impedance matrix.

Due to the continuity constraints imposed via (5.18), the size of the CLCN system matrix in (5.58) is reduced compared to the original LCN impedance matrix for all orders 
of the LCN discretization. For closed geometries, $N_{e}=2 N_{c}$. In this case, the memory reduction factor (MRF), defined as the ratio of the size of the CLCN matrix to the LCN matrix for the EFIE formulation,

$$
\operatorname{MRF}_{\text {EFIE }}=\left(\frac{N_{T}}{2 N_{c} N_{q}}\right)^{2}=\left(\frac{p+1}{p+2}\right)^{2} .
$$

For a zeroth-order LCN discretization of a closed geometry using mixed-order bases, the MRF is 0.25 , which corresponds to a memory savings of four relative to the original LCN system.

Based on (3.54), the CLCN discretization of the EFIE can be represented as:

$$
\left[\mathrm{Z}^{\mathrm{CLCN}}\right] \bar{I}=\bar{V}
$$

where, $\left[\mathrm{Z}^{\text {CLCN }}\right]$ is the CLCN system matrix defined in (5.58), and $\bar{V}$ is a vector of size $N_{T} \times 1$, which can be expressed as:

$$
\bar{V}=[v]^{T}\left[\mathrm{~L}^{\mathrm{s}}\right] \bar{f},
$$

where $\bar{f}$ was defined in (3.55). $\bar{I}$ in (5.60) is a vector of size $N_{T} \times 1$, comprising all CLCN DOFs associated with cells and edges. Upon solving (5.60), the LCN current coefficients are recovered as:

$$
\overline{\mathrm{j}}=[v]\left[\mathrm{L}^{\mathrm{s}}\right]^{T} \bar{I} .
$$

The CLCN discretization is thus comprised of the system (5.60), with the matrix, excitation and unknown definitions provided by (5.58), (5.61), and (5.62). The new elements in these definitions (relative to the original LCN method) are simply right singular vectors of the constraint conditions (5.21) and (5.30), which are given by (5.56) and (5.57). Thus, in practice, the CLCN method consists of defining appropriate 
constraints on the underlying LCN basis functions and using a singular value decomposition to extract the requisite transformation vectors from the right singular vectors of the constraint matrix. In the present case, this process and its interpretation has been discussed in detail for high-, mixed-order Legendre bases on quadrilateral cells.

\subsection{Numerical Results}

To evaluate the CLCN discretization method, we consider its application to EFIE, augmented EFIE, and MFIE formulations for a number of PEC objects having smooth or singular geometries.

\subsubsection{CLCN applied to the EFIE}

We start by investigating the scattering from a one-meter radius PEC sphere illuminated by a $-\hat{\mathbf{z}}$ traveling plane wave polarized along the $\hat{\mathbf{x}}$ direction. The surface of the sphere is discretized with 96 sixth-order quadrilateral cells. The integration tolerances for all the following examples are chosen 1e-6. Figure 12 displays the RMS errors in the bi-static radar cross-sections (RCS) at $300 \mathrm{MHz}$ and $50 \mathrm{MHz}$ obtained using CLCN discretization of the EFIE compared to the errors from the LCN discretization. The errors are plotted versus basis order, $p$. The RMS errors are computed as:

$$
\operatorname{RMS} \operatorname{Error}(x)=\sqrt{\frac{\sum_{i=1}^{N}\left|x^{c a l}\left(\theta_{i}\right)-x^{r e f}\left(\theta_{i}\right)\right|^{2}}{\sum_{i=1}^{N}\left|x^{r e f}\left(\theta_{i}\right)\right|^{2}}}
$$


where $x^{\text {cal }}\left(\theta_{i}\right)$ and $x^{\text {ref }}\left(\theta_{i}\right)$ refer respectively to the calculated and the reference solutions at $\theta_{i}\left(\theta_{i}=0^{\circ}, 1^{\circ}, \ldots, 180^{\circ}\right)$. For scattering from the sphere, the Mie series is used as the reference solution.

From Figure 12, it is observed that both CLCN and LCN discretizations exhibit similar exponential convergence behaviors. For orders zero and one, the RCS errors are similar, but the CLCN matrices are 4 and 2.25 times smaller than the respective LCN matrices. Figure 13 displays the condition numbers corresponding to the system matrices underlying the data in Figure 12. The results show that the CLCN method lowers the rate of increase of the condition numbers versus order as compared to the LCN method for the EFIE formulation.

In order to demonstrate the effectiveness of the CLCN discretization for singular geometries, consider scattering from a PEC cube with a one-meter edge length for an $\hat{\mathbf{x}}$ polarized, $-\hat{\mathbf{z}}$ traveling incident plane wave at $50 \mathrm{MHz}$. The cube surface is meshed by 54 square patches. The RCS in the $\phi=0^{\circ}$ plane obtained using the CLCN and LCN discretizations of the EFIE for $p=0$ and $p=1$ are shown in Figure 14. The RCS obtained using the RWG MOM on a mesh with 5540 triangular facets is included as a reference solution. The relative RMS differences (computed using (5.63)) between the reference RWG solution and the RCS obtained using the CLCN and LCN systems are also displayed in Figure 14. Enforcing normal continuity of currents with the CLCN method markedly improves the RCS for the cube geometry at these relatively low orders.

In order to examine the CLCN discretization method when applied to an open geometry, we consider scattering from the PEC corner reflector previously described in [36]. The corner reflector is meshed with 243 square patches. The excitation is a $50 \mathrm{MHz}$ 
plane wave incident from the $\theta=\pi / 4, \varphi=\pi / 4$ direction; the linearly polarized electric field points in the $0.5 \hat{\mathbf{x}}+0.5 \hat{\mathbf{y}}-0.707 \hat{\mathbf{z}}$ direction. Figure 15 displays the RCS of the corner reflector in the $\phi=0^{\circ}$ plane computed by the CLCN and LCN discretizations of the EFIE for $p=0$ and $p=1$; the results are compared to a reference solution obtained using an RWG MOM discretization with a fine mesh containing 17304 triangular cells. As observed above for the cube, there is a discernible discrepancy between the RCS given by the zeroth-order LCN discretization and the reference solution, which is substantially diminished when the CLCN discretization is used. Moreover, referring to Figure 15, the RCS of the corner reflector computed by the first order CLCN discretization demonstrates improved accuracy compared to the corresponding LCN solution. The number of DOFs for the zeroth and first order LCN discretizations are 972 and 2916; there are 459 and 1890 DOFs for the corresponding CLCN discretizations.

\subsubsection{CLCN applied to the augmented EFIE}

In this section, the CLCN method is used with the augmented EFIE (AEFIE) formulation. As it was mentioned, the AEFIE formulation was invented to address the low frequency breakdown of the EFIE. The AEFIE formulation incorporates the charge as additional unknown and includes the current continuity constraints. The continuity constraint on the surface of the cell is represented as [31]:

$$
\nabla . J(\mathbf{r})=-j \omega q_{s}(\mathbf{r}),
$$

where, $q_{s}$ is the surface charge. The continuity equation on the lth edge shared by the cells $n_{1}$ and $n_{2}$ is reduced to: 


$$
-\mathbf{e}_{n 1}(\mathbf{r}) \cdot \mathbf{J}_{n 1}(\mathbf{r})-\mathbf{e}_{n 2}(\mathbf{r}) \cdot \mathbf{J}_{n 2}(\mathbf{r})=-j \omega q_{l}(\mathbf{r})
$$

where $\mathbf{e}_{n 1}$ and $\mathbf{e}_{n 2}$ are used to indicate the outward vectors emanating from the cells $n_{1}$ and $n_{2}$ that are normal to the edge shared by the two cells at the point indicated by the vector $\mathbf{r} . q_{l}$ is the line charge. Considering the $\mathrm{LCN}$ discretization of the $\mathbf{L}$ operator discussed in chapter 3, the EFIE formulation is expressed as:

$$
\begin{aligned}
\mathbf{t} \cdot \mathbf{E}^{\text {inc }}(\mathbf{r})=j k_{0} \eta_{0} \mathbf{t} \cdot \sum_{\text {cells }} \int_{S_{c}} \mathbf{J}\left(\mathbf{r}^{\prime}\right) G & \left(\mathbf{r}, \mathbf{r}^{\prime}\right) d s^{\prime} \\
& +j k_{0}^{-1} \eta_{0} \sum_{\text {cells }} \int_{S_{c}}\left(\mathbf{t} \cdot \nabla G\left(\mathbf{r}, \mathbf{r}^{\prime}\right)\right)\left[\nabla_{\|}^{\prime} \cdot \mathbf{J}\left(\mathbf{r}^{\prime}\right)\right] d s^{\prime} \\
& -j k_{0}^{-1} \eta_{0} \sum_{\text {cells }} \int_{\partial S_{c}} \mathbf{e}^{\prime} \cdot \mathbf{J}\left(\mathbf{r}^{\prime}\right)\left(\mathbf{t} \cdot \nabla G\left(\mathbf{r}, \mathbf{r}^{\prime}\right)\right) d l^{\prime},
\end{aligned}
$$

where, $S_{c}$ is the surface of the cell; $\partial S_{c}$ is the contour encircling $S_{c} . \mathbf{e}^{\prime}$ is the outward normal to the $\partial S_{c} \cdot \nabla_{\|}^{\prime} \cdot$ is the surface divergence. Applying (5.64) and (5.65), (5.66) is represented as:

$$
\begin{aligned}
\mathbf{t} \cdot \mathbf{E}^{\text {inc }}(\mathbf{r})=\eta_{0}\left[\mathbf{t} \cdot \sum_{\text {cells } \int_{c}} j k_{0} \mathbf{J}\left(\mathbf{r}^{\prime}\right)\right. & G\left(\mathbf{r}, \mathbf{r}^{\prime}\right) d v^{\prime} \\
& +\sum_{\text {cells }} \int_{S_{c}}\left(\mathbf{t} \cdot \nabla G\left(\mathbf{r}, \mathbf{r}^{\prime}\right)\right) c_{0} q_{s}\left(\mathbf{r}^{\prime}\right) d v^{\prime} \\
& \left.+\sum_{\text {edges } I_{c}} \int_{(\mathbf{t}}\left(\mathbf{t} \cdot \nabla G\left(\mathbf{r}, \mathbf{r}^{\prime}\right)\right) c_{0} q_{l}\left(\mathbf{r}^{\prime}\right) d s^{\prime}\right],
\end{aligned}
$$

where, $c_{0}$ is the speed of light. Applying $j k_{0} \mathbf{J}, c_{0} q_{v}$, and $c_{0} q_{s}$ as unknowns the matrix form of the AEFIE including (5.67), (5.64), and (5.65) is demonstrated as:

$$
\left[\begin{array}{ccc}
\mathbf{L}_{A} & \mathbf{L}_{q_{s}} & \mathbf{L}_{q_{l}} \\
\mathbf{D}_{s} & -k_{0}^{2} \mathbf{I} & 0 \\
\mathbf{D}_{l} & 0 & -k_{0}^{2} \mathbf{I}
\end{array}\right] \cdot\left[\begin{array}{c}
j k_{0} \overline{\mathrm{j}} \\
c_{0} \overline{\mathrm{q}}_{s} \\
c_{0} \overline{\mathrm{q}}_{l}
\end{array}\right]=\frac{1}{\eta_{0}}\left[\begin{array}{c}
\overline{\mathrm{f}} \\
0 \\
0
\end{array}\right] .
$$


where, $\overline{\mathrm{j}}$ and $\overline{\mathrm{f}}$ are column vectors defined in (3.55). A mixed order representation of currents with $p(p+1)$ samples in each unitary direction is utilized. $\bar{q}_{s}$ and $\bar{q}_{l}$ include the samples of surface and line charges represented by order $p$ polynomial complete Legendre basis functions. The quantities of the system matrix in (5.68) are defined based on (5.64) to (5.67) . $\left[\mathbf{D}_{s}\right]$ and $\left[\mathbf{D}_{l}\right]$ are respectively related to the continuity equation on the cell surface and edges (5.64) and (5.65). $\left[\mathbf{L}_{A}\right]$ corresponds to the contribution of the vector potential to the EFIE; $\left[\mathbf{L}_{q_{s}}\right]$ and $\left[\mathbf{L}_{q_{l}}\right]$ are associated with the scalar potential term in the EFIE. [I] refers to the identity matrix. It should be noted that the charge neutrality constraint is replaced by one row in the system matrix of (5.68) to have a full rank system matrix. The charge neutrality constraint states that some of all charges are zero [31]:

$$
\sum_{N_{c}} \int_{S_{c}} c_{0} q_{s}\left(\mathbf{r}^{\prime}\right) d s^{\prime}+\sum_{N_{e}} \int_{l_{e}} c_{0} q_{l}\left(\mathbf{r}^{\prime}\right) d l^{\prime}=0
$$

where, $N_{c}$ and $N_{e}$ are the number of cells and edges respectively. In this section, the CLCN method is used with the augmented EFIE (AEFIE) formulation. Because of the discontinuity of the currents across the edges, the LCN discretization of the AEFIE requires the incorporation of the line charge DOFs in the formulation. Applying the transformation matrix developed for the EFIE, the CLCN discretization of the AEFIE system matrix is depicted as:

$$
\left[\mathrm{Z}_{\mathrm{A}}^{\mathrm{CLCN}}\right]=\left[v_{\mathrm{A}}\right]^{T} \cdot\left[\mathrm{Z}_{\mathrm{ANQL}}^{\mathrm{LCN}}\right] \cdot\left[v_{\mathrm{A}}\right]
$$


where, $\left[\mathrm{Z}_{\mathrm{ANQL}}^{\mathrm{LCN}}\right]$ is the LCN discretized AEFIE system matrix without the inclusion of line charge DOFs:

$$
\left[\mathrm{Z}_{\mathrm{ANQL}}^{\mathrm{LCN}}\right]=\left[\begin{array}{cc}
\mathbf{L}_{A} & \mathbf{L}_{q_{s}} \\
\mathbf{D}_{s} & -k_{0}^{2} \mathbf{I}
\end{array}\right] .
$$

and,

$$
v_{\mathrm{A}}=\left[\begin{array}{cc}
{\left[v_{\mathrm{B}}\right]} & {[0]} \\
{[0]} & {[\mathrm{I}]}
\end{array}\right],
$$

where,

$$
\left[v_{\mathrm{B}}\right]=\left[\mathrm{L}^{\mathrm{s}}\right]^{T}[v]
$$

$[v]$ is the transformation matrix defined in (5.55) . In (5.70), the continuity of currents are imposed through multiplication of $\left[v_{B}\right]$ from the right side by the blocks of the LCN discretized AEFIE that are associated with the current DOFs, i.e. $\mathbf{L}_{A}$ and $\mathbf{D}_{s}$. Similarly, the continuity of the vector test quantities in (5.70) is enforced by multiplication of $\left[v_{\mathrm{B}}\right]^{T}$ from the left side by $\left[\mathbf{L}_{A}\right]$ and $\left[\mathbf{L}_{q_{s}}\right]$ which correspond to the EFIE.

For closed geometries, the memory savings factor (MSF) provided by the CLCN discretization of the AEFIE vis-à-vis an LCN discretization is:

$$
\mathrm{MSF}_{\mathrm{AEFIE}}=\left(\frac{3 p+3}{3 p+7}\right)^{2}
$$

In the following, we discuss the results of the CLCN discretization of the AEFIE in (5.70) applied to the test cases studied in the previous section. Figure 16 illustrates the RMS errors in the RCS for the one-meter radius PEC sphere at $300 \mathrm{MHz}$ obtained using 
the CLCN method with the AEFIE; the errors obtained from the corresponding LCN discretization with and without line charge are also shown.

The errors in the near electric field for the PEC sphere at $1 \mathrm{~Hz}$ are reported in Figure 17 versus basis order. The near electric fields are computed on a semi-circle with a radius of 1.5 meters. The results of Figure 16 and Figure 17 demonstrate that the CLCN method applied to the AEFIE converges exponentially by increasing the basis order, and the accuracy is similar to that obtained using the LCN method with line charge. The CLCN discretization, which does not include line charge, offers considerably higher accuracy than the LCN method without line charge. Figure 18 represents the condition numbers of the system matrices resulting from the CLCN and LCN methods applied to AEFIE for the cases studied in Figure 16 and Figure 17. The CLCN method considerably decreases the rate of growth of the condition numbers compared to the standard LCN discretization at both $1 \mathrm{~Hz}$ and $300 \mathrm{MHz}$.

The cross-section obtained using a CLCN discretization of the AEFIE with the corner reflector discussed above are shown in Figure 19 at a frequency of $50 \mathrm{MHz}$. The corner reflector is meshed with 243 square patches. Results obtained using the standard LCN discretization are also shown. Both CLCN and LCN results are compared to the reference RWG solution discussed above. The CLCN method provides significantly better accuracy compared to the LCN results obtained both with and without the incorporation of line charges.

Finally, Figure 20 displays the near electric scattered field at $1 \mathrm{~Hz}$ computed using the CLCN and LCN discretizations of the AEFIE for the corner reflector problem. The corner reflector is meshed with 147 square patches. The reference solution obtained using 
the RWG MOM on a fine mesh is also shown. The electric field is computed on a semicircle of radius 2 meters in the $\phi=0^{\circ}$ plane. The electric field solutions from the LCN discretization of the AEFIE without line charge demonstrate a large discrepancy with respect to the reference solution. The CLCN method provides more accurate near electric fields than the LCN method while also reducing the size of system matrix.

\subsubsection{CLCN applied to the MFIE}

In this section CLCN is applied to discretize the magnetic field integral equation (MFIE). As it was mentioned in chapter 3, the reciprocal unitary vectors are the appropriate choice to discretize the MFIE since they diagonalize the corresponding operator. Referring to (5.8), $\mathbf{T}_{m}^{k}(\mathbf{r})$ is decomposed in reciprocal unitary vector directions [50]:

$$
\mathbf{T}_{m}^{k}(\mathbf{r})=T_{m}^{k, 1}(\mathbf{r}) \mathbf{a}^{1}(\mathbf{r})+T_{m}^{k, 2}(\mathbf{r}) \mathbf{a}^{2}(\mathbf{r})
$$

Therefore, (5.9) is rewritten as:

$$
\int_{S_{m}} \mathbf{T}_{m}^{k}(\mathbf{r}) \cdot \mathbf{f}^{i n c}(\mathbf{r}) d s \approx \sum_{i=1}^{2} \sum_{q=1}^{N_{q}} \omega_{q_{m}}^{i} \mathbf{T}_{m}^{k, i}(\mathbf{r}) \cdot \mathbf{f}^{i n c}\left(\mathbf{r}_{q_{m}}^{i}\right) .
$$

where, $\mathbf{T}_{m}^{k, i}(\mathbf{r})=T_{m}^{k, i}(\mathbf{r}) \mathbf{a}^{i}\left(\mathbf{r}^{i}\right)$. Similar to the CLCN discretization of the EFIE, the local constraints are developed by choosing the mixed order Legendre polynomials as the test basis functions. Thus, considering (3.91) and (3.92) we have:

$$
\begin{gathered}
\mathbf{T}_{m}^{k, 1}=\frac{P_{n}\left(u^{1}\right) P_{l}\left(u^{2}\right)}{\sqrt{g_{s}}} \mathbf{a}^{1}, \quad(n=0, \ldots, p+1 ; l=0, \ldots, p) \\
\mathbf{T}_{m}^{k, 2}=\frac{P_{n}\left(u^{1}\right) P_{l}\left(u^{2}\right)}{\sqrt{g_{s}}} \mathbf{a}^{2}, \quad(n=0, \ldots, p ; l=0, \ldots, p+1)
\end{gathered}
$$


Similar to (5.23) and (5.33), the elements of the cell and edge local constraint matrices related to imposition of the normal continuity on the test basis functions are defined as:

$$
\left[\mathrm{C}_{m}^{j}\right]_{b q, k}=\mathbf{e}_{m}\left(\mathbf{r}_{b q_{m}}\right) \cdot \mathbf{T}_{m}^{k, j}\left(\mathbf{r}_{b q_{m}}\right)
$$

The transformation vectors associated with the test side are then obtained based on the new constraint matrices.

In this section the results of scattering from the $1 \mathrm{~m}$ radius PEC sphere obtained by using CLCN and LCN discretizations of the MFIE are presented. Mixed-order Legendre basis functions are used. Reciprocal unitary vectors are used as test vectors for the MFIE. The surface of the sphere is modeled by 96 sixth order quad cells, and the incident wave is a $\hat{\mathbf{x}}$ polarized plane wave in $-\hat{\mathbf{z}}$ direction at $200 \mathrm{MHz}$. Figure 21 demonstrates exponential convergence of the RMS errors in the radar cross section (RCS) obtained using CLCN and LCN discretizations of the EFIE and MFIE formulations versus basis order. The condition numbers of the system matrices examined in Figure 21 are provided in Figure 22. It is observed that the CLCN-MFIE provides condition numbers that are stable with respect to increasing basis order while the LCN-MFIE condition numbers are not stable versus basis order. 


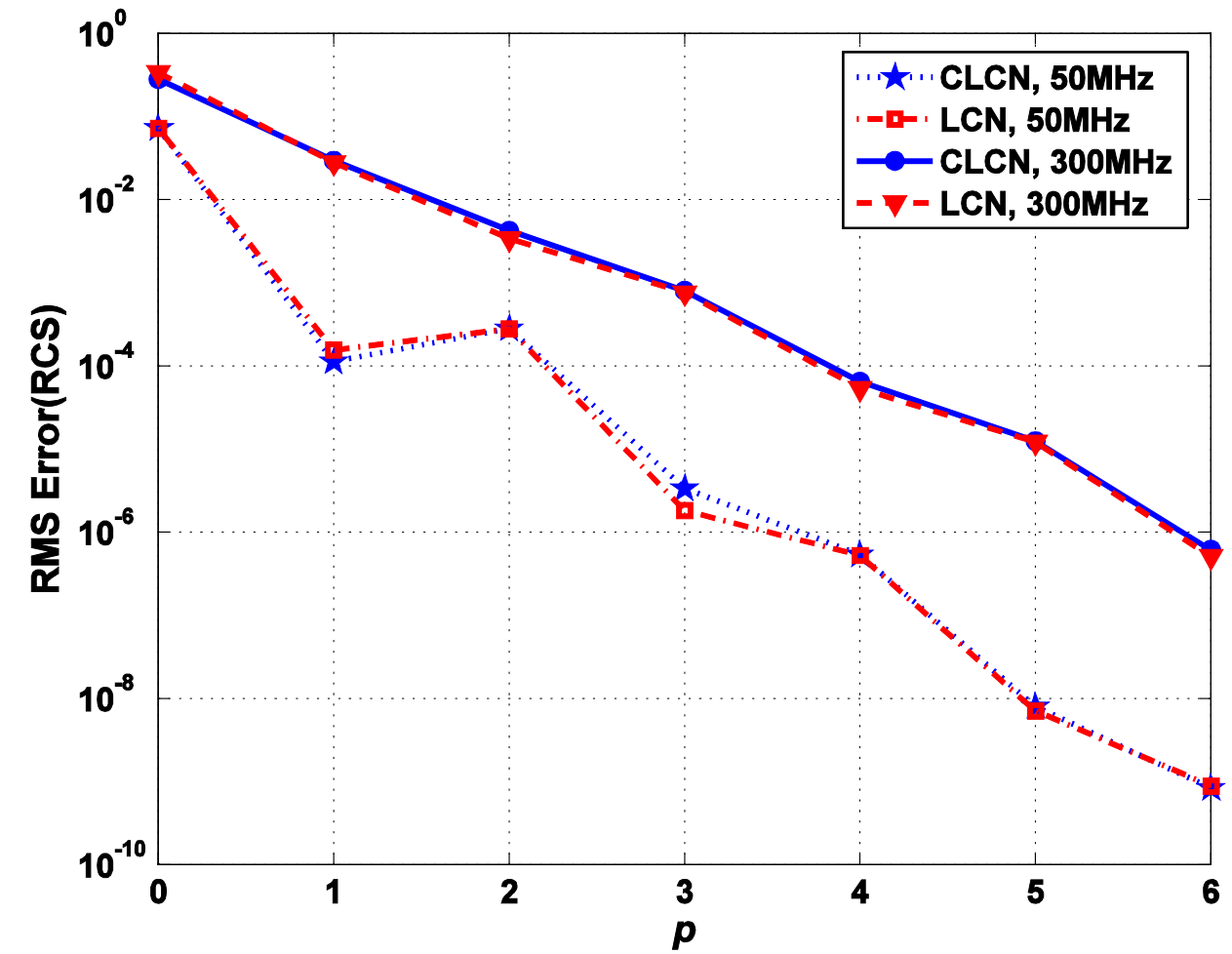

Figure 12: RMS error of the bi-static RCS for the 1-meter radius PEC sphere at $50 \mathrm{MHz}$ and $300 \mathrm{MHz}$ versus the basis order. 


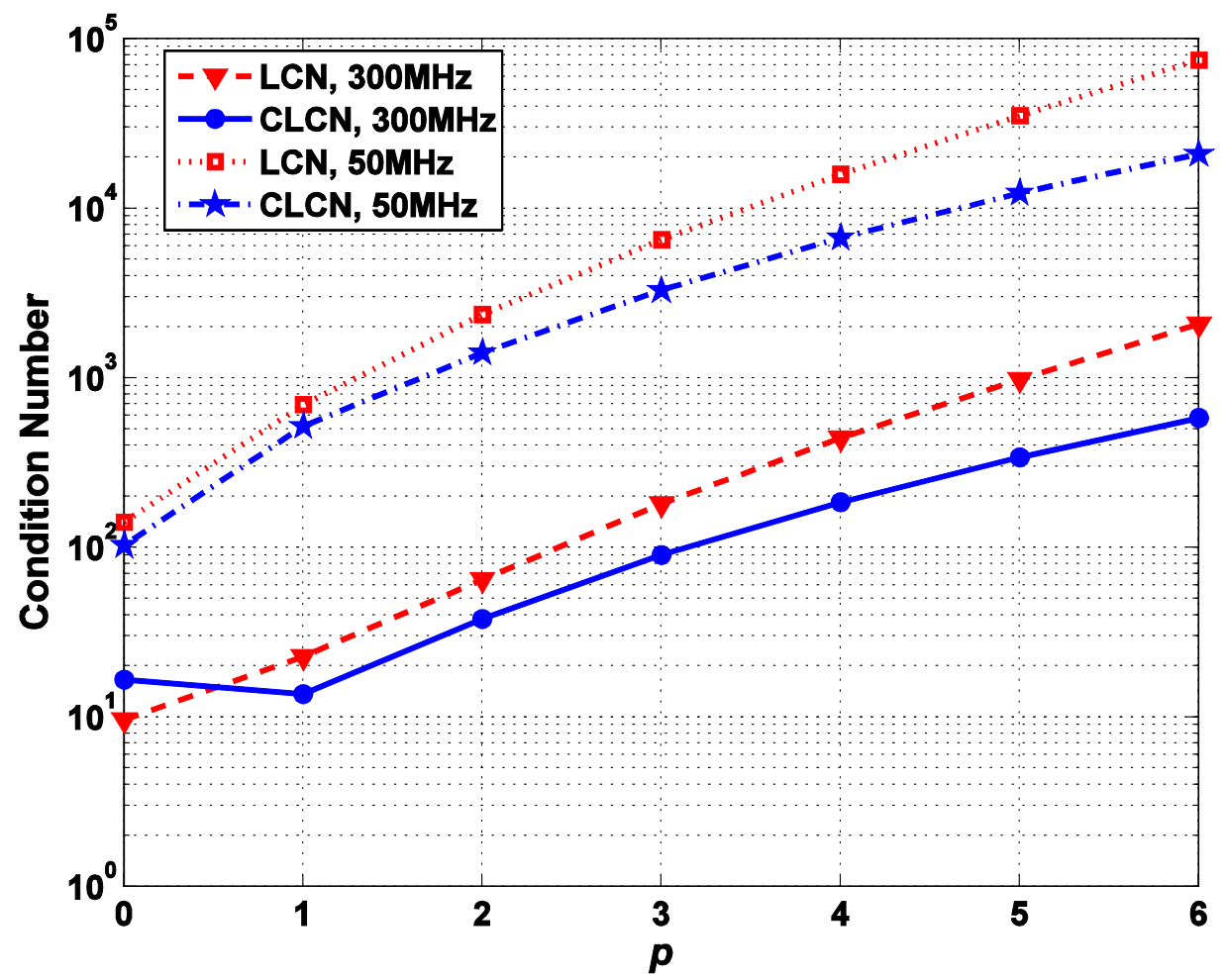

Figure 13: Condition numbers for the 1-meter radius PEC sphere at $50 \mathrm{MHz}$ and $300 \mathrm{MHz}$ versus the basis order. 


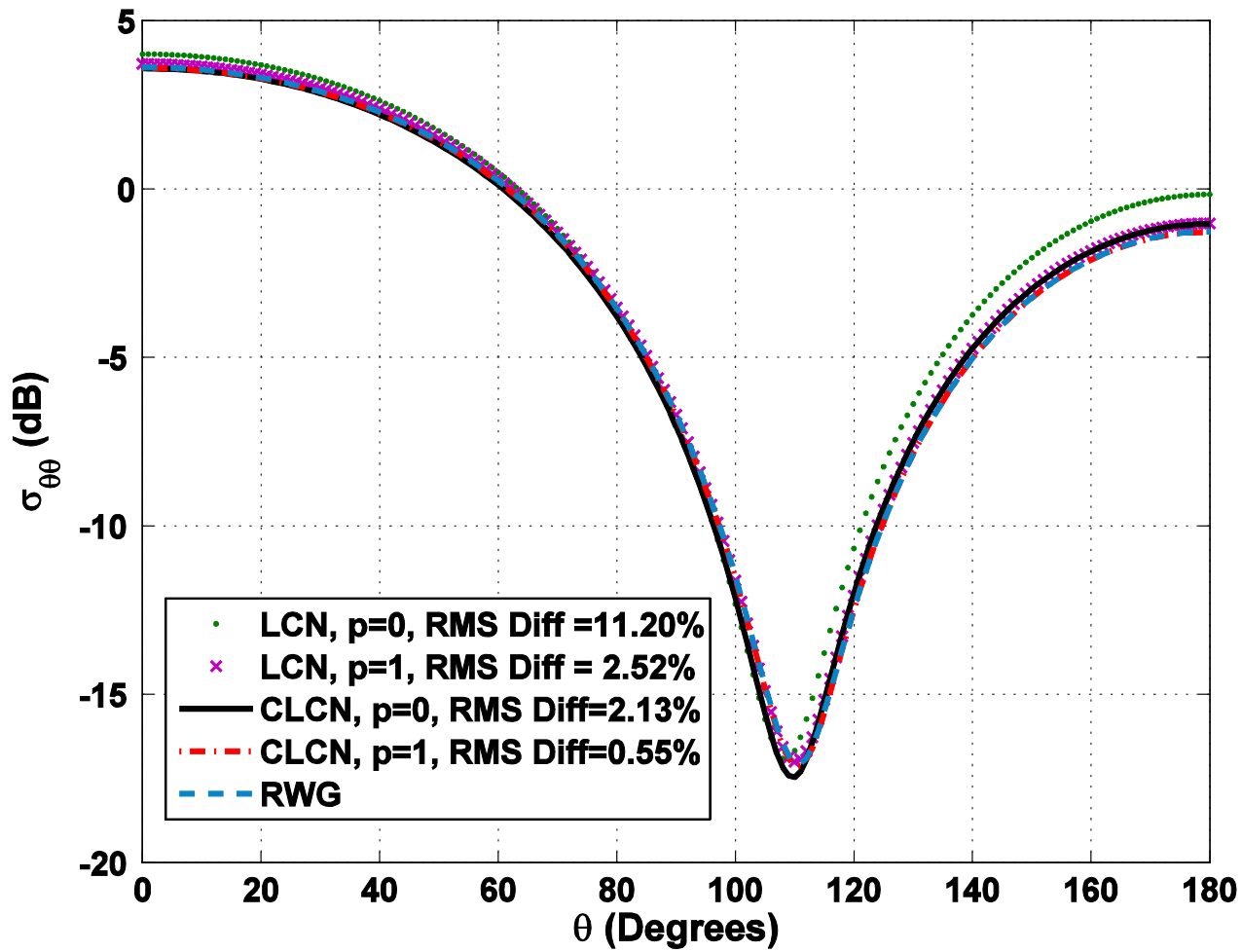

Figure 14: Radar cross-sections for scattering from a PEC cube with a 1-meter edge length at $50 \mathrm{MHz}$ in the $\phi=0$ plane. 


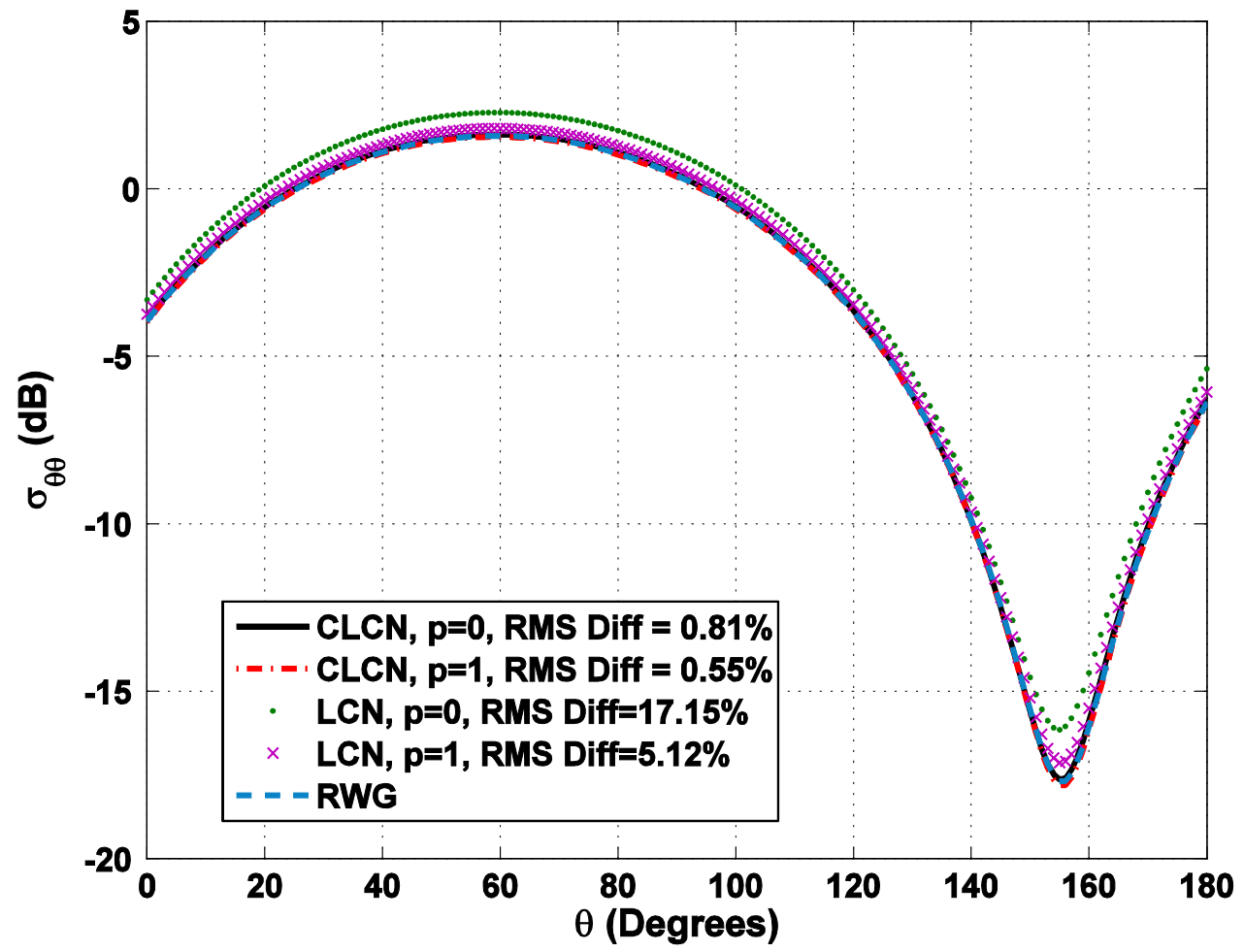

Figure 15: Radar cross-sections for scattering from a PEC corner reflector at $50 \mathrm{MHz}$ in the $\phi=0^{\circ}$ plane. 


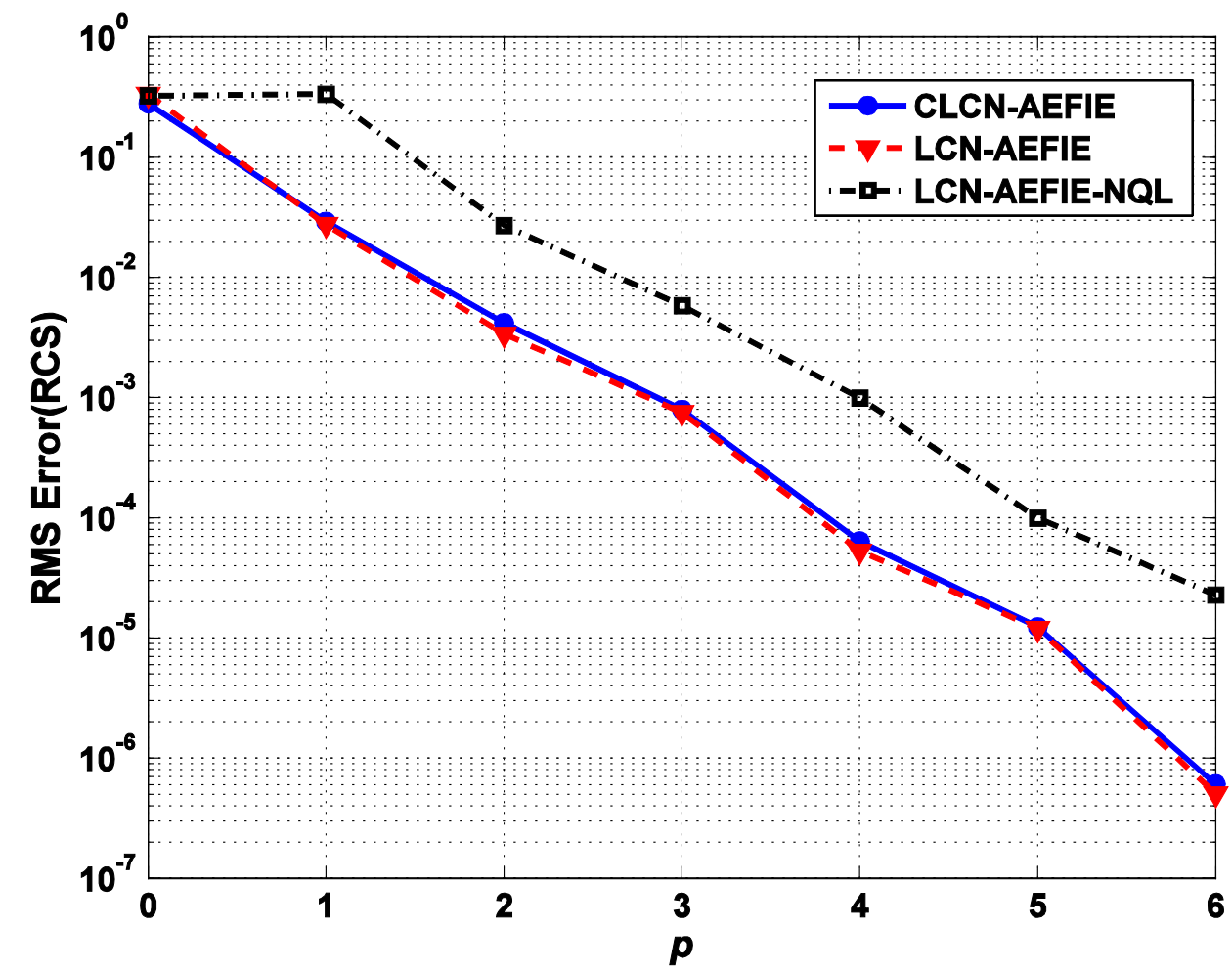

Figure 16: RMS error of the bi-static RCS for the 1-meter radius PEC sphere at $300 \mathrm{MHz}$ versus the basis order. In the legend, NQL indicates no line charge. 


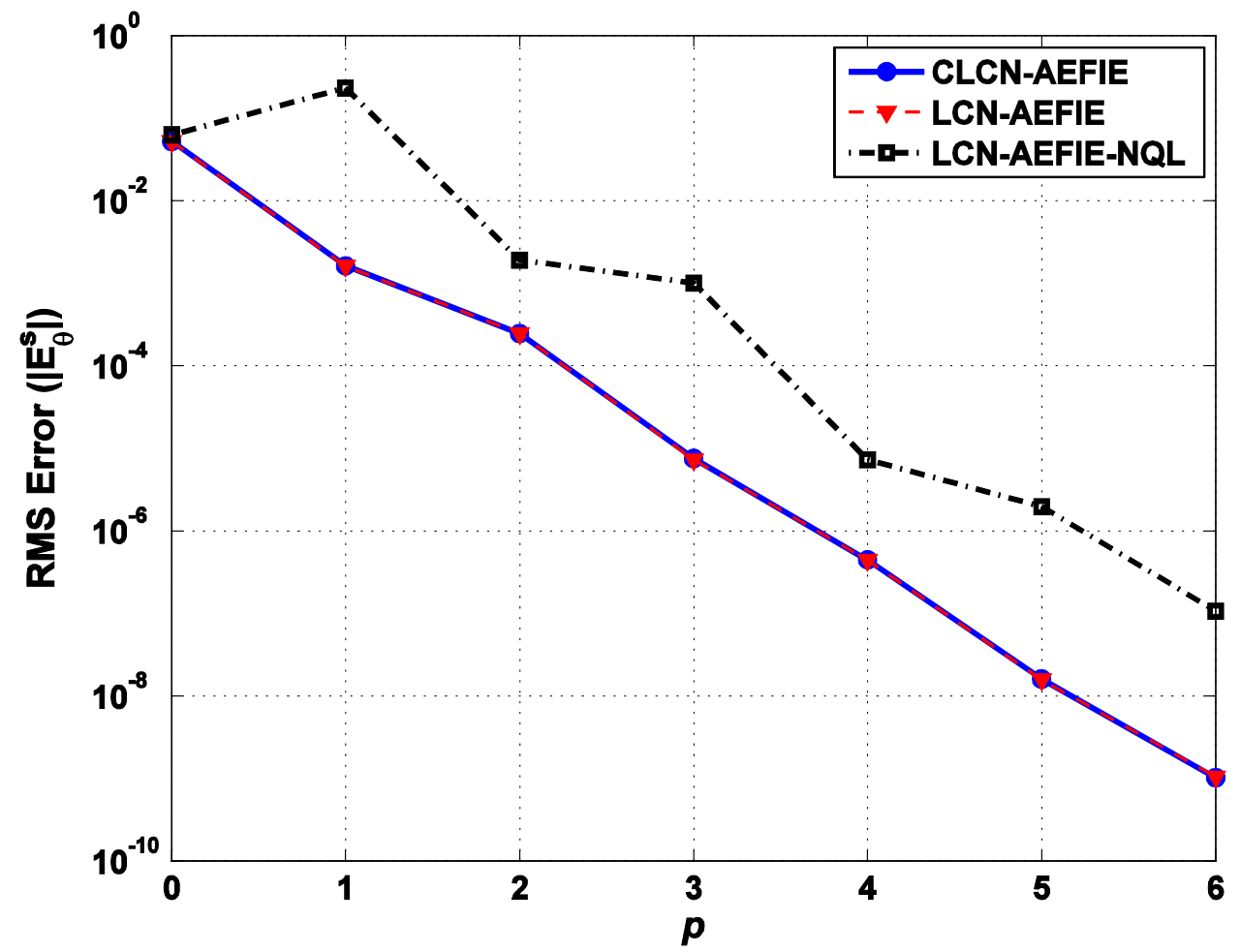

Figure 17: RMS error of the near electric field for the 1-meter radius PEC sphere at $1 \mathrm{~Hz}$ versus basis order $p$. The near field is sampled $0.5 \mathrm{~m}$ above the PEC surface. In the legend, NQL indicates no line charge. 


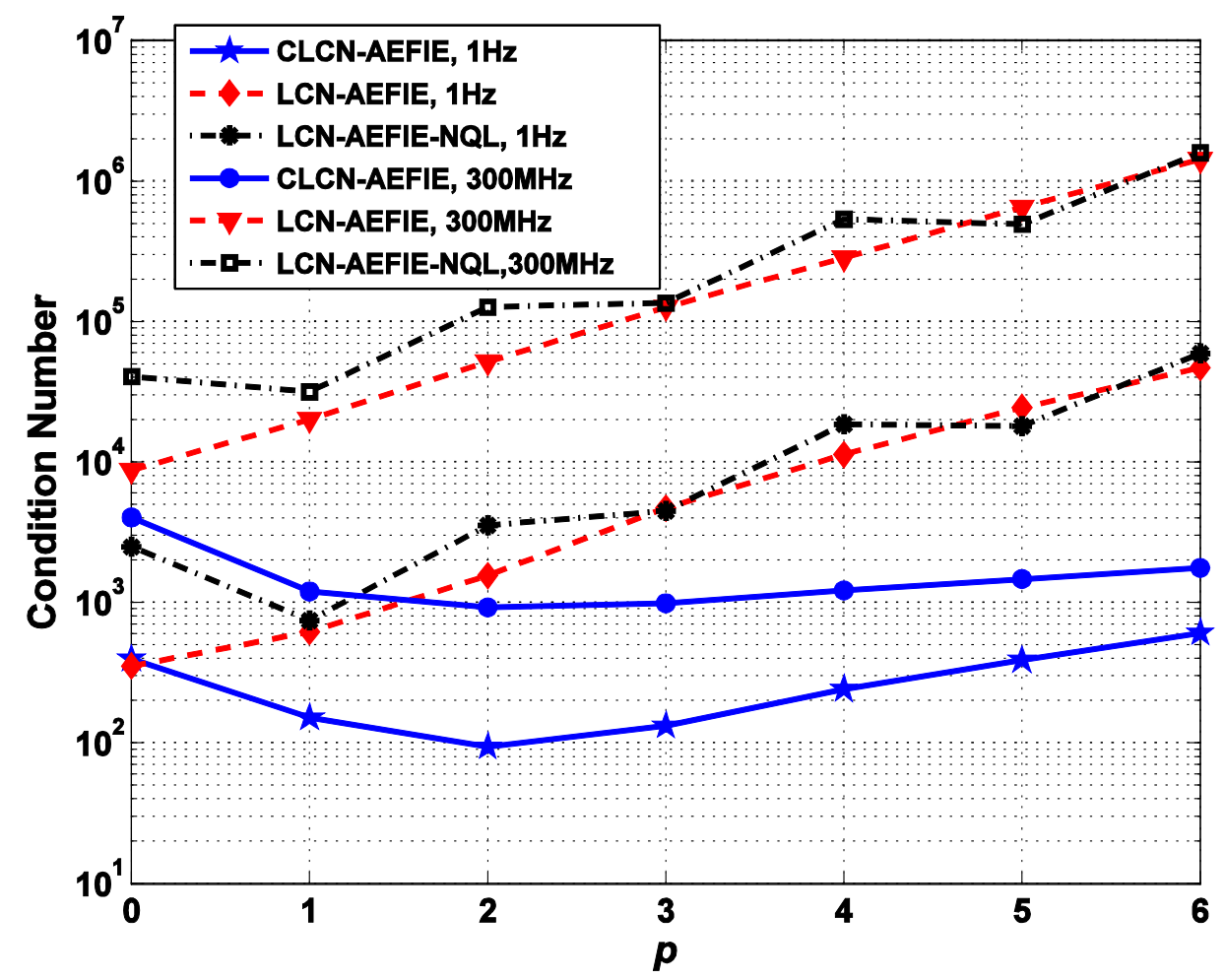

Figure 18: Condition numbers of the CLCN and LCN system matrices obtained for the AEFIE applied to a 1-meter radius PEC sphere at $300 \mathrm{MHz}$ and $1 \mathrm{~Hz}$ versus the basis order. 


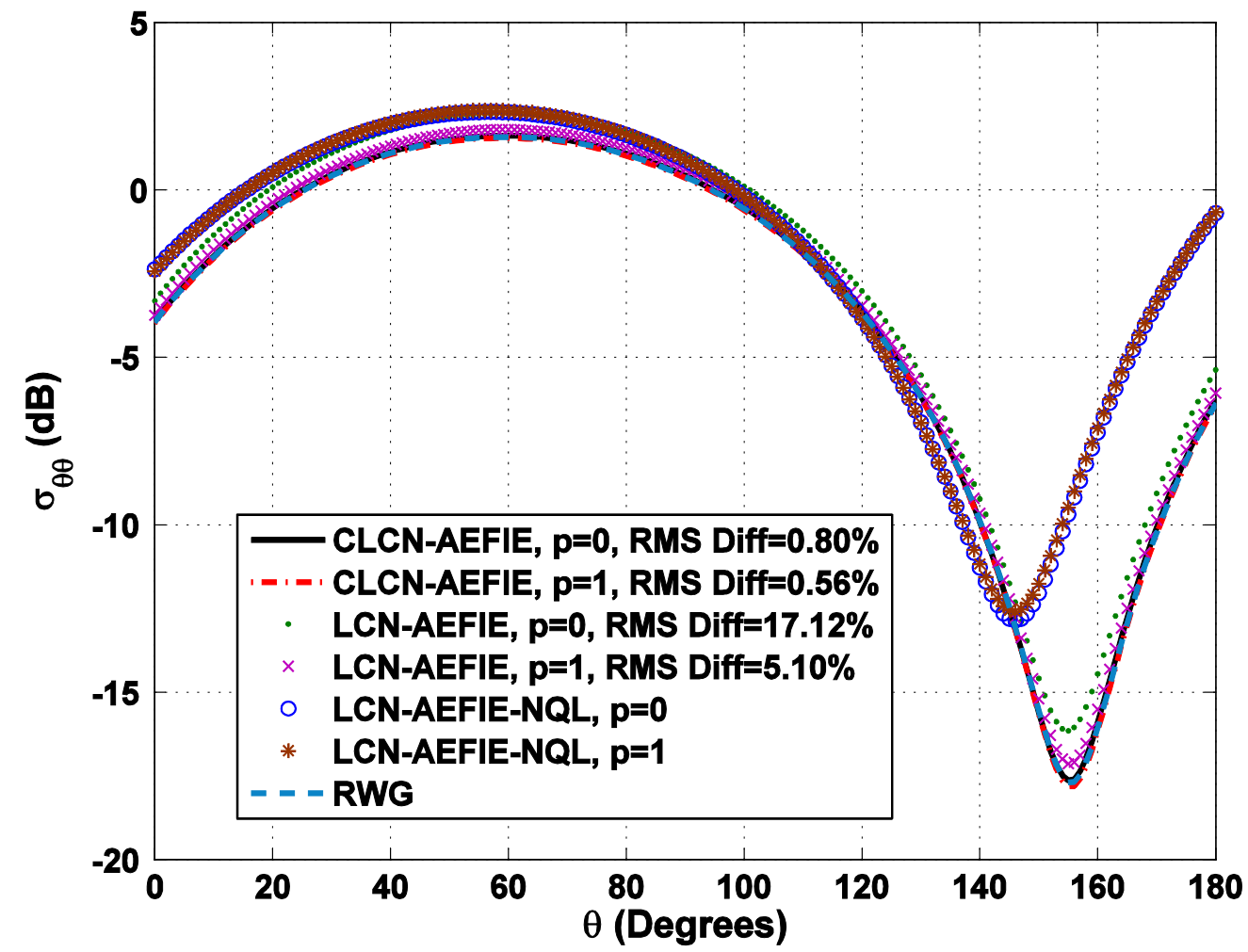

Figure 19: Radar cross-sections for scattering from a PEC corner reflector at $50 \mathrm{MHz}$ in the $\phi=0$ plane . 


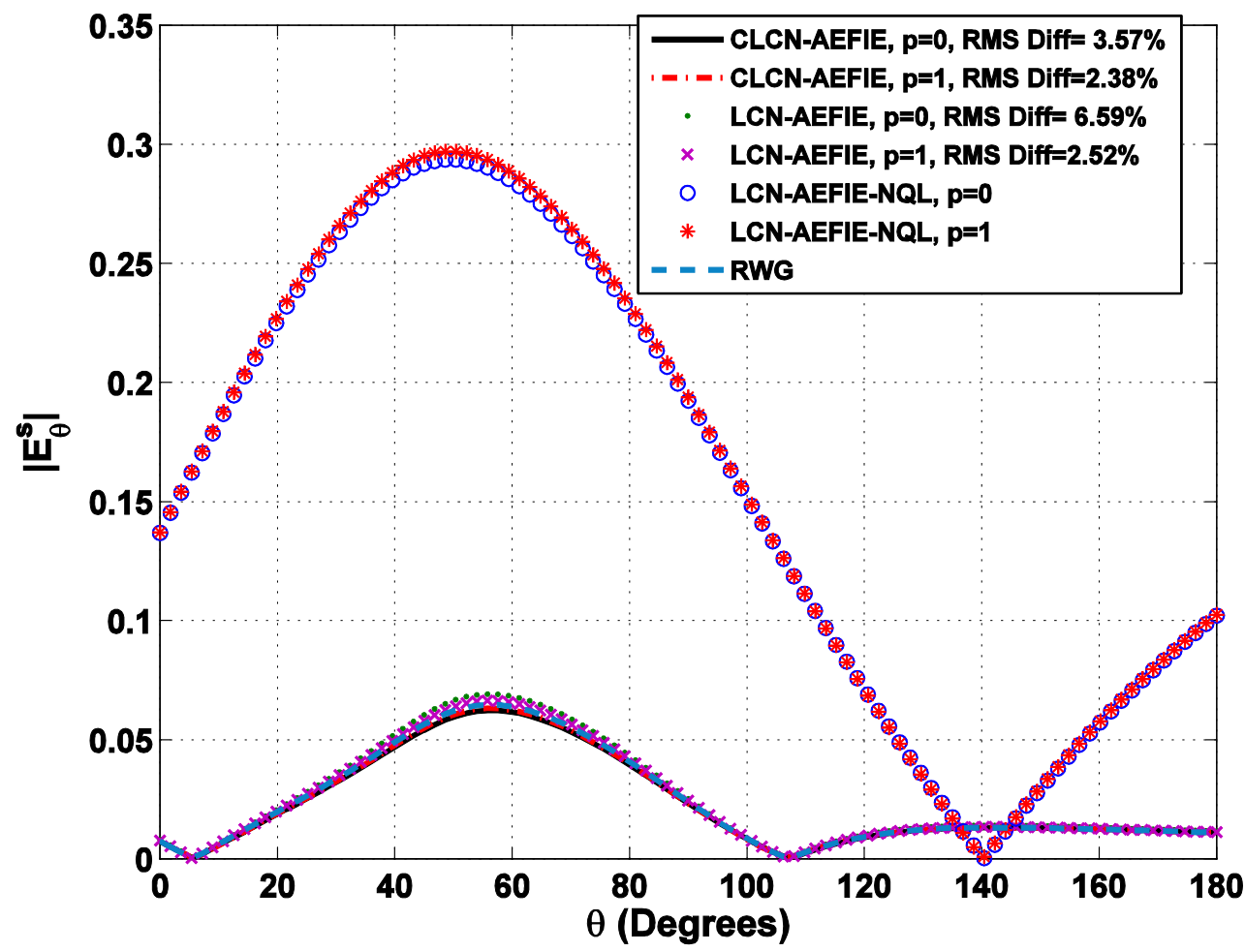

Figure 20: Near electric field for the corner reflector at $1 \mathrm{~Hz}$ sampled on a two meter radius semi-circle in the $\phi=0$ plane 


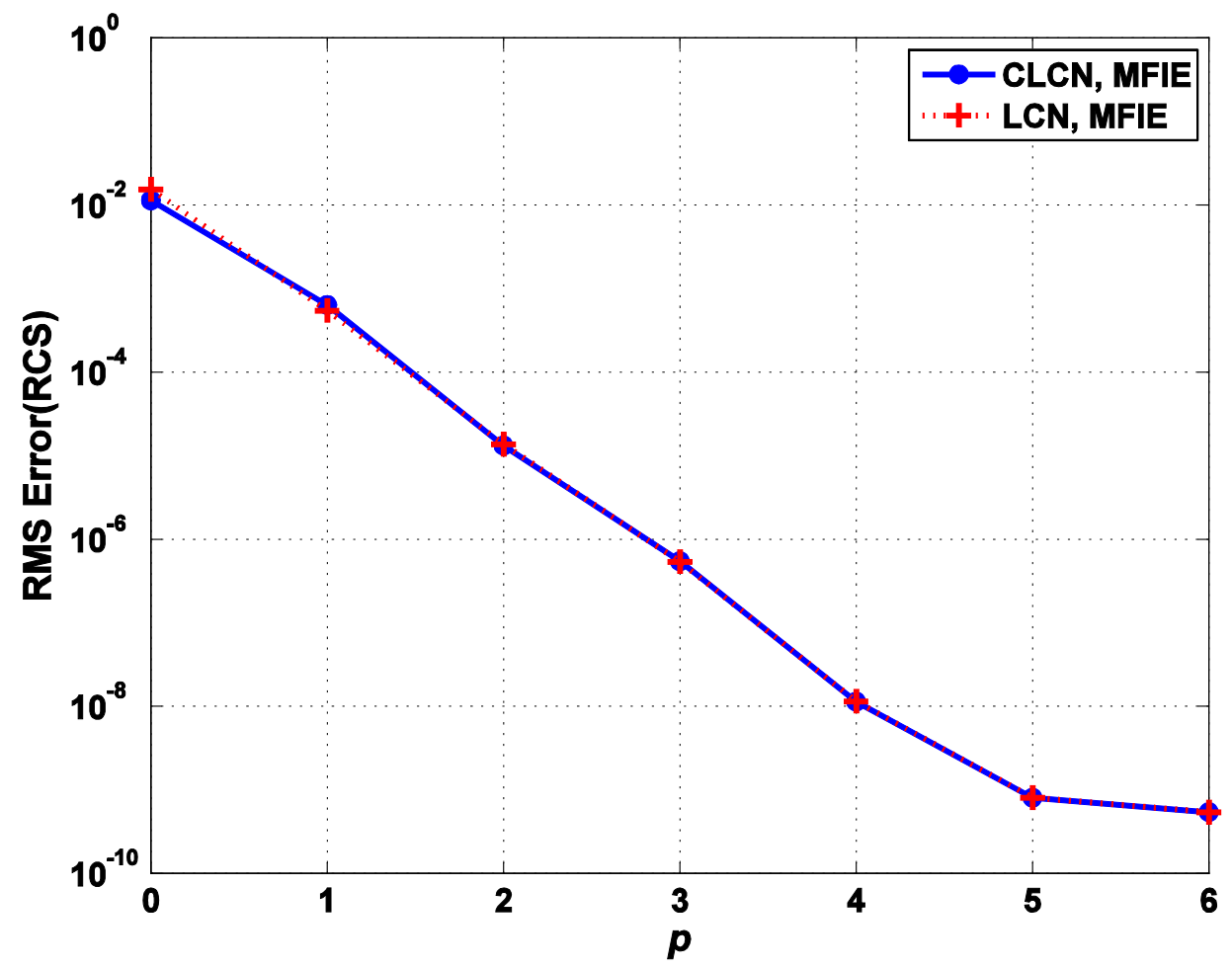

Figure 21: RMS error of the bi-static RCS for the 1-meter radius PEC sphere at $200 \mathrm{MHz}$ versus the basis order. 


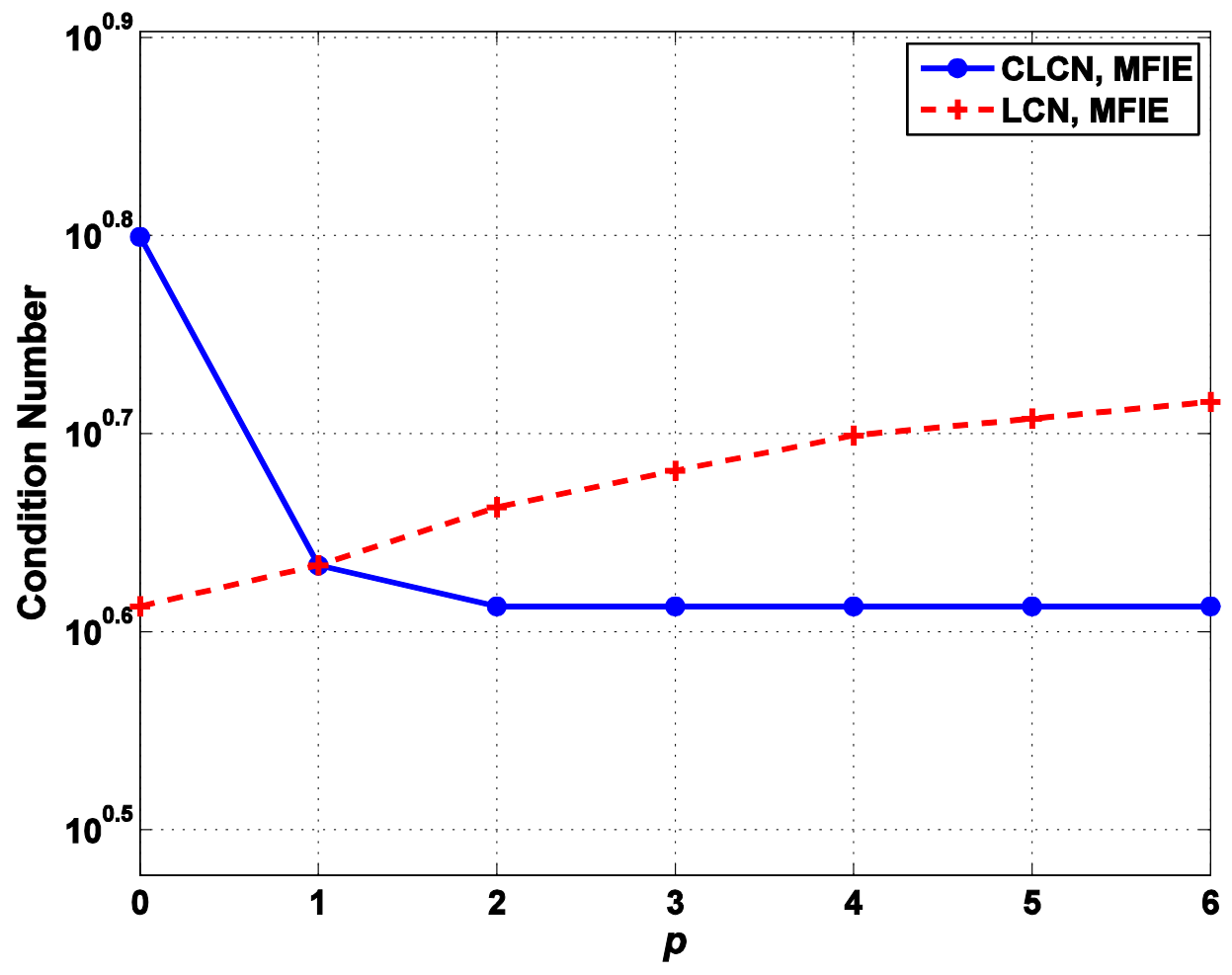

Figure 22: Condition numbers of the CLCN and LCN system matrices obtained for the MFIE applied to a 1-meter radius PEC sphere at $200 \mathrm{MHz}$ versus the basis order.

Copyright (C) Nastaran Hendijani 2015 


\section{Chapter 6. ConCLUSIONS}

A high-order, constrained LCN (CLCN) discretization method has been developed and tested. The method provides a general extension of the high-order LCN method that is obtained by imposing auxiliary constraints on the underlying LCN basis. Instead of mimicking a particular MOM discretization, the CLCN method is based on the construction of cell- and edge-based subspaces that satisfy specific continuity constraints. The CLCN method does not require the specification of any of the underlying CLCN basis functions; the only basis functions sampled are those of the LCN method [28].

The auxiliary CLCN constraints were herein used to enforce normal continuity on the LCN basis. It was shown that the right singular vectors obtained from the auxiliary continuity constraint matrices provide the necessary transformations of both the domain and the range of the LCN system matrix. As a result, the CLCN system matrix is simply a product of the original LCN system matrix with sparse left and right matrices; the only samples required of the underlying kernels are those required by the original LCN method. In this way, the high-order CLCN method provides computational advantages similar to those provided by the high-order LCN method.

The CLCN method has been applied to the EFIE, MFIE, and AEFIE formulations of scattering from perfect electrical conductors. The CLCN method was observed to reduce the size of the system matrix relative to the original LCN matrix in all cases. It was also shown that the size of the memory savings decreases as the order of the discretization increases.

For smooth geometries, the accuracy obtained from the CLCN method is similar to that provided by the LCN method. In the case of a sphere, the high-order convergence of 
the CLCN method is essentially similar to that obtained from the original LCN method at both high and low frequencies.

For singular geometries, the CLCN method provides better accuracy in both the near and far fields for orders $p=0$ and $p=1$. In the case of an AEFIE formulation, the CLCN system matrix is better conditioned than the LCN system matrix as the order increases. It was also observed that the CLCN method does not require the use of line charge DOF in discretizing the AEFIE. This provides significant simplification and computational savings relative to the LCN method which requires the use of line charge DOF for accurate discretization of the AEFIE.

\subsection{Contributions}

The following items are the contributions of the work comprised in this dissertation:

- A high-order, constrained LCN (CLCN) discretization method has been developed and tested. The method enforces normal continuity on the underlying LCN source and test quantities by imposing auxiliary constraints on the underlying LCN basis. Local transformation vectors are simply developed from the right singular vectors of a homogeneous system of auxiliary constraints:

$\checkmark$ High-order implementation is straightforward. There is no need to construct the HO basis functions explicitly.

$\checkmark$ The additional computational time for implementing the constraints matrices and forming the local transformation vectors is negligible

$\checkmark$ Extension of the CLCN to general mesh elements is straightforward. 
$\checkmark$ Compared to the LCN, the CLCN method provides smaller system matrices for all underlying formulations.

$\checkmark$ The CLCN method offers better conditioning of the system matrix. Conditioning of CLCN-MFIE is order-stable, while LCN-MFIE does not provide stable condition numbers by increasing the basis order. In addition, CLCN-EFIE and CLCN-AEFIE are better conditioned than LCN counterparts.

$\checkmark$ The CLCN formulation removes the need to include the line integrals or line charges in the formulation. This feature was validated for the AEFIE as it was observed that CLCN-AEFIE without line charges provides as accurate solutions as the LCN-AEFIE with line charges.

$\checkmark$ It was observed that for coarse meshes and/or low orders the CLCN method provides better accuracy for objects with sharp edges than the LCN.

- A new volume integral equation (VIE) based formulation called augmented VIE (AVIE) was proposed and developed. The AVIE formulation relies on the inclusion of surface and volume charge continuity constraints. It has been demonstrated that unlike the VIE formulation, the AVIE provides stable condition numbers by increasing the basis order at low frequencies and provides accurate solutions for the electric and magnetic fields at both high and low frequencies. To further improve the conditioning of the AVIE, an iterative left and right diagonal scaling preconditioner was implemented. It was observed that the iterative preconditioner improves the condition number 
of the AVIE system matrix significantly, while similar attempts to scale the regular VIE do not significantly improve the condition numbers of the corresponding system matrix.

\subsection{Future Work}

- The CLCN method in this dissertation was implemented to discretize surface integral equations with quadrilateral mesh elements. The method can also be implemented with triangular mesh elements. Moreover, the extension of the CLCN to the volume integral equations is an area of future work. It is expected that the CLCN-AVIE improves the eigenvalue pattern of the LCNAVIE for applications involving dense iterative solvers.

- In this work, the mixed order Legendre polynomial functions have been applied as test and source basis functions. It is useful to investigate the application of order complete Legendre polynomials as the CLCN basis functions.

- The CLCN method is intended to be utilized to solve large problems in conjunction with OL-LOGOS factorization algorithm. Due to the point based structure of the CLCN and the local imposition of the normal continuity constraints, the implementation looks straightforward.

Copyright (C) Nastaran Hendijani 2015 


\section{REFERENCES}

[1] A. F. Peterson, S. L. Ray, and R. Mittra, Computational methods for electromagnetics. Piscataway, NJ: IEEE Press, 1998.

[2] J. Jin, The finite element method in electromagnetics: John Wiley \& Sons, 2014.

[3] P. Monk and P. Monk, Finite element methods for Maxwell's equations: Clarendon Press Oxford, 2003.

[4] R. F. Harrington, Field computation by moment methods. New York,: Macmillan, 1968.

[5] L. Greengard, J. Huang, V. Rokhlin, and S. Wandzura, "Accelerating fast multipole methods for the Helmholtz equation at low frequencies," Computational Science \& Engineering, IEEE, vol. 5, pp. 32-38, 1998.

[6] F. X. Canning and K. Rogovin, "Simply sparse, a general compression/solution method for MoM programs," in Antennas and Propagation Society International Symposium, 2002. IEEE, 2002, pp. 234-237.

[7] R. J. Adams, Y. Xu, X. Xu, J. S. Choi, S. D. Gedney, and F. X. Canning, "Modular fast direct electromagnetic analysis using local-global solution modes," IEEE Transactions on Antennas and Propagation, vol. 56, pp. 2427-2441, Aug 2008.

[8] X. Xu and R. J. Adams, "Sparse matrix factorization using overlapped localizing LOGOS modes on a shifted grid," IEEE Transactions on Antennas and Propagation, vol. 60, pp. 1414-1424, 2012.

[9] Y. Xu, X. Xu, R. J. Adams, S. D. Gedney, and F. X. Canning, "Sparse direct solution of the electric field integral equation using nonoverlapped localizing logos modes," Microwave and Optical Technology Letters, vol. 50, pp. 303-307, Feb 2008.

[10] J. Cheng and R. J. Adams, "Direct solution method using overlapped localizing LOGOS modes for AEFIE-G at low frequencies," in 28th International Review of Progress in Applied Computational Electromagnetics, pp. 579-584, Columbus, $\mathrm{OH}, 2012$.

[11] J. A. Stratton, Electromagnetic theory, 1st ed. New York, London,: McGraw-Hill book company, inc., 1941.

[12] B. N. Datta, Numerical linear algebra and applications: Siam, 2010.

[13] G. H. Golub and C. F. Van Loan, Matrix computations vol. 3: JHU Press, 2012.

[14] H. A. Van der Vorst, "Bi-CGSTAB: A fast and smoothly converging variant of Bi-CG for the solution of nonsymmetric linear systems," SIAM Journal on scientific and Statistical Computing, vol. 13, pp. 631-644, 1992.

[15] Y. Saad, Iterative methods for sparse linear systems: Siam, 2003.

[16] M. M. Kostic and B. M. Kolundzija, "Maximally orthogonalized higher order bases over generalized wires, quadrilaterals, and hexahedra," Antennas and Propagation, IEEE Transactions on, vol. 61, pp. 3135-3148, 2013.

[17] F. X. Canning and K. Rogovin, "Fast direct solution of standard moment-method matrices," Antennas and Propagation Magazine, IEEE, vol. 40, pp. 15-26, 1998. 
[18] R. D. Graglia, D. R. Wilton, and A. F. Peterson, "Higher order interpolatory vector bases for computational electromagnetics," Antennas and Propagation, IEEE Transactions on, vol. 45, pp. 329-342, 1997.

[19] E. Jorgensen, J. L. Volakis, P. Meincke, and O. Breinbjerg, "Higher order hierarchical Legendre basis functions for electromagnetic modeling," Antennas and Propagation, IEEE Transactions on, vol. 52, pp. 2985-2995, 2004.

[20] L. R. Hamilton, J. J. Ottusch, M. A. Stalzer, R. S. Turley, J. L. Visher, and S. M. Wandzura, "Numerical solution of 2-D scattering problems using high-order methods," Antennas and Propagation, IEEE Transactions on, vol. 47, pp. 683691, 1999.

[21] M. Djordjevic and B. M. Notaros, "Double higher order method of moments for surface integral equation modeling of metallic and dielectric antennas and scatterers," Antennas and Propagation, IEEE Transactions on, vol. 52, pp. 21182129, 2004.

[22] L. F. Canino, J. J. Ottusch, M. A. Stalzer, J. L. Visher, and S. M. Wandzura, "Numerical solution of the Helmholtz equation in 2D and 3D using a high-order Nystrom discretization," Journal of Computational Physics, vol. 146, pp. 627-663, Nov 11998.

[23] S. D. Gedney, "On Deriving a Locally Corrected Nyström Scheme from a Quadrature Sampled Moment Method," IEEE Transactions on Antennas and Propagation, vol. 51, pp. 2402-2412, September 2003.

[24] S. D. Gedney, A. Zhu, and C. C. Lu, "Study of mixed-order basis functions for the locally-corrected Nyström method," IEEE Transactions on Antennas and Propagation, vol. 52, pp. 2996-3004, November 2004.

[25] A. M. Zhu, S. D. Gedney, and J. L. Visher, "A study of combined field formulations for material scattering for a locally corrected Nystrom discretization," IEEE Transactions on Antennas and Propagation, vol. 53, pp. 4111-4120, Dec 2005.

[26] S. M. Rao, D. Wilton, and A. W. Glisson, "Electromagnetic scattering by surfaces of arbitrary shape," Antennas and Propagation, IEEE Transactions on, vol. 30, pp. 409-418, 1982.

[27] S. M. Rao, A. W. Glisson, D. R. Wilton, and B. S. Vidula, "A simple numerical solution procedure for statics problems involving arbitrary-shaped surfaces," IEEE Transactions on Antennas and Propagation, vol. 27, pp. 604-608, 1979.

[28] N. Hendijani, J. Cheng, R. J. Adams, and J. C. Young, "Constrained Locally Corrected Nyström Method," accepted to IEEE Transactions on Antennas and Propagation, 2015.

[29] A. F. Peterson, "Accuracy of currents produced by the Locally-corrected Nyström method and the Method of Moments when used with higher-order representations," Applied Computational Electromagnetics Society Journal, vol. 17, pp. 74-83, March 2002.

[30] M. Shafieipour, I. Jeffrey, J. Aronsson, and V. I. Okhmatovski, "On the Equivalence of RWG Method of Moments and the Locally Corrected Nyström Method for Solving the Electric Field Integral Equation," Antennas and Propagation, IEEE Transactions on, vol. 62, pp. 772-782, 2014. 
[31] J. C. Young, Y. Xu, R. J. Adams, and S. D. Gedney, "High-order Nyström implementation of an agumented electric field integral equation," IEEE Antennas and Wireless Propagation Letters, vol. 11, pp. 846-849, 2012.

[32] P. Yla-Oijala, J. Markkanen, S. Jarvenpaa, and S. P. Kiminki, "Surface and Volume Integral Equation Methods for Time-Harmonic Solutions of Maxwell's Equations," Progress In Electromagnetics Research, vol. 149, pp. 15-44, 2014.

[33] N. Hendijani, R. J. Adams, and J. C. Young, "High-order Nyström implementation of an augmented volume integral equation," in Radio Science Meeting (Joint with AP-S Symposium), 2014 USNC-URSI, 2014, pp. 187-187.

[34] P. A. Knight and D. Ruiz, "A fast algorithm for matrix balancing," IMA Journal of Numerical Analysis, p. drs019, 2012.

[35] J. Cheng, "Formulation and Solution of Electromagnetic Integral Equations Using Constraint-Based Helmholtz Decompositions," PhD Dissertation, 2012.

[36] J. Cheng and R. J. Adams, "Electric field-based surface integral constraints for Helmholtz decompositions of the current on a conductor," Antennas and Propagation, IEEE Transactions on, vol. 61, pp. 4632-4640, 2013.

[37] R. J. Adams, "Physical and analytical properties of a stabilized electric field integral equation," Antennas and Propagation, IEEE Transactions on, vol. 52, pp. 362-372, 2004.

[38] F. P. Andriulli, K. Cools, H. Bagci, F. Olyslager, A. Buffa, S. Christiansen, et al., "A Multiplicative Calderon Preconditioner for the Electric Field Integral Equation," Antennas and Propagation, IEEE Transactions on, vol. 56, pp. 23982412, 2008.

[39] G. Vecchi, "Loop-star decomposition of basis functions in the discretization of the EFIE," IEEE Transactions on Antennas and Propagation, vol. 47, pp. 339-346, 1999.

[40] N. Hendijani, J. Cheng, and R. J. Adams, "Combined Field Integral Equation Using a Constraint-Based Helmholtz Decomposition," Antennas and Propagation, IEEE Transactions on, vol. 62, pp. 1500-1503, 2014.

[41] N. Hendijani, J. Cheng, and R. Adams, "Nystrom implementation of a combined field integral equation using approximate helmholtz decompositioncs of the current on a PEC," in Radio Science Meeting (Joint with AP-S Symposium), 2013 USNC-URSI, 2013, pp. 107-107.

[42] J. R. Mautz and R. F. Harrington, "An E-field solution for a conducting surface small or comparable to the wavelength," IEEE Transactions on Antennas and Propagation, vol. 32, pp. 330-339, 1984.

[43] J. S. Zhao and W. C. Chew, "Integral equation solution of Maxwell's equations from zero frequency to microwave frequencies," IEEE Transactions on Antennas and Propagation, vol. 48, pp. 1635-1645, Oct 2000.

[44] E. J. Nyström, "Über die praktische Auflüsung von Integral-gleichungen mit Anwendungen auf Randwertaufgaben," Acta Mathematica, vol. 54, pp. 185-204, 1930.

[45] R. Mittra, Computational Electromagnetics: Recent Advances and Engineering Applications: Springer Science \& Business Media, 2013.

[46] M. G. Duffy, "Quadrature over a pyramid or cube of integrands with a singularity at a vertex," SIAM journal on Numerical Analysis, vol. 19, pp. 1260-1262, 1982. 
[47] J. Markkanen, "Discrete Helmholtz Decomposition for Electric Current Volume Integral Equation Formulation," 2014.

[48] T.-Y. Chen and J. W. Demmel, "Balancing sparse matrices for computing eigenvalues," Linear Algebra and Its Applications, vol. 309, pp. 261-287, 2000.

[49] N. Hendijani, J. Cheng, R. J. Adams, and J. C. Young, "High Order Locally Corrected Nyström Method with Continuity Constraints," in The Applied Computational Electromagnetics Society (ACES), Williamsburg, VA, 2015.

[50] N. Hendijani, J. Cheng, R. J. Adams, and J. C. Young, "High Order Locally Corrected Nyström Method with Normal Continuity," in AP-S Symposium, 2015 APS/URSI, 2015. 


\section{Vita}

Nastaran Hendijani obtained the high school diploma from the National Center for Exceptionally Talented Students, Tehran in August of 1998. She received the B.S. degree in Electrical Engineering from Amirkabir University of Technology (Tehran Polytechnic), Tehran in September of 2003. In September of 2006, she received the M.S. degree in Electrical Engineering from Iran University of Science and Technology (IUST), Tehran. From August of 2009 to January of 2012 she was a graduate research assistant at Virginia Tech, Blacksburg, VA. Since January of 2012, she has been working as a graduate research assistant at University of Kentucky, Lexington, KY. 